\title{
Universal objects in categories of reproducing kernels
}

Daniel Beltiţă and José E. Galé

\begin{abstract}
We continue our earlier investigation on generalized reproducing kernels, in connection with the complex geometry of $C^{*}$ - algebra representations, by looking at them as the objects of an appropriate category. Thus the correspondence between reproducing $(-*)$-kernels and the associated Hilbert spaces of sections of vector bundles is made into a functor. We construct reproducing $(-*)$-kernels with universality properties with respect to the operation of pull-back. We show how completely positive maps can be regarded as pull-backs of universal ones linked to the tautological bundle over the Grassmann manifold of the Hilbert space $\ell^{2}(\mathbb{N})$.
\end{abstract}

\section{Introduction}

The present work belongs to a line of research which has been initiated in the papers [5], [4], [3], and concerns representation theory for infinitedimensional Lie groups on the one hand, and an approach to operator algebras by means of techniques from differential geometry on the other hand. In [5], geometric realizations of GNS representations on groups of unitaries in $C^{*}$-algebras are obtained, in the spirit of the Bott-Borel-Weil theorem. Hilbertian section spaces of these realizations, in that infinite-dimensional setting, are constructed using the tool of reproducing kernels on vector bundles. As regards holomorphy, there are a few cases of unitary groups (or homogeneous spaces on which those groups act) where such a structural

2000 Mathematics Subject Classification: Primary: 46E22; Secondary: 47B32, 46L05, 18A05, 58B12.

Keywords: Reproducing kernel, category theory, vector bundle, tautological bundle, Grassmann manifold, completely positive map, universal object. 
property appears automatically; see [5], and also [4] for (homogeneous) orbits of Grassmannians. In order to extend the theory of [5] to general holomorphic actions of complex Lie groups on complexified vector bundles, and to incorporate the unitary case to the wider setting of complex geometry, a new kind of generalized reproducing kernels on complex vector bundles has been introduced in [3], that takes into account prescribed involutions of the bundle bases. (In passing, the theory enables us to present a holomorphic geometric view of Stinespring dilations of completely positive maps.)

In this way, we find the notion of (generalized) reproducing kernels on vector bundles in the core of that part of (infinite-dimensional) representation theory which is suitable to be treated using methods of complex geometry. So it seems to be a demanding task to study generalized reproducing kernels in themselves, mainly looking at those aspects of them which are more directly related to the aforementioned subjects. One of the relevant facts emerging naturally in the subject is that Grassmannian manifolds, over the Hilbert spaces defined by the kernels, seem to be the proper place where all the elements of the geometric theory are settled in a canonical manner; see [4]. Since Grassmannians arise naturally in geometry as a universal notion, we wonder if they can be also considered as the universal model for the geometrical implications of reproducing kernels. This question is further supported by the feeling that - loosely speaking - reproducing kernels are very much alike (so that, in particular for the classical ones, their mutual differences are mainly confined to function theory).

In order to make this idea precise, we continue here our earlier investigation on generalized reproducing kernels by organizing them as a category and looking for the corresponding universal objects. Thus the correspondence between reproducing $(-*)$-kernels (see the definition below) and the associated Hilbert spaces will be looked at as a functor. By following this categorial approach we find that there exist reproducing $(-*)$-kernels enjoying universality properties with respect to the operation of pull-back. In particular it turns out that all of the reproducing kernels (in the classical sense, that is, for scalar valued functions) arise as pull-backs of a universal reproducing kernel that lives on the tautological bundle over the Grassmann manifold of the Hilbert space $\ell^{2}(\mathbb{N})$ (see Theorem 6.2 and the comment preceding it).

In a purely mathematical direction, investigations on operations on reproducing kernels have been previously carried out, with motivation coming from complex analysis, function theory, learning theory, and other areas; some recent references are [1] [17], [18], and [7]. The theory of kernels in the scalar case was systematically developed in [2]. An extension to vector valued functions has been worked out in [19], as well as a systematic account of this theory is included in [23]. Reproducing kernels for Hilbert spaces 
of sections generalize the above classes. There are already present in [16], but the first framework where to consider kernels for Hilbert spaces of sections of (finite-dimensional) complex bundles seems to have been suggested in [6]. The corresponding extension to the setting of vector bundles with infinite-dimensional fibers of infinite-dimensional manifolds is done in [5]. The article [3] extends in turn the results of [5] to reproducing kernels associated with involutive diffeomorphisms in the bundle base spaces.

Reproducing kernels on line bundles over finite-dimensional complex manifolds have been on the other hand considered in quantum mechanics as well, related to quantization of classical states; see [24], [25] and [22], as well as some references therein. Roughly speaking, in the model proposed in [24] and [25] a mechanical system is identified with a triple

$$
(Z, \mathcal{H}, \zeta: Z \rightarrow \mathbf{C P}(\mathcal{H}))
$$

where $Z$ is a complex manifold seen as the phase space of classical states of the system, $\mathcal{H}$ is a Hilbert space and $\mathbf{C P}(\mathcal{H})$ is its corresponding complex projective space, which is to play the role of the phase space of pure quantum states. Then the (geometric) quantization of the classical phase space $Z$ is supplied by the mapping $\zeta$. It turns out that the transition probability amplitudes linked to the phase space $Z$ can be formalized in a way that gives rise to a reproducing kernel $K$ defined on a complex line bundle with base $Z$. Then the quantization map $\zeta=\zeta_{K}$ is constructed out of the kernel $K$, through the reproducing kernel Hilbert space $\mathcal{H}_{K}$ associated with $K$, see [25] for details. The mapping $\zeta$ is very important in this approach since under certain conditions it replaces the calculation of the Feynman integral which expresses the transition amplitudes between states. Moreover, it is possible to recover the reproducing kernel defining the transition probabilities directly from $\zeta$.

In fact, the relationship between $K$ and $\zeta$ admits an interpretation in a categorial framework such as it has been developed in the paper [25], with a view toward establishing a canonical relation between the classical observables and the quantum ones (see also [24]). In this setting, natural ways of viewing states of the matter take the form of three different categories (in two of them the objects are complex line bundles, with a distinguished kernel $K$ in one case and a distinguished Hilbert space $\mathcal{H}$ in the other; in the third one the objects are the mappings $\zeta$ ) and it is possible to show the mutual functorial equivalence between them, see [25], p. 387. Pull-backs of the tautological universal bundle with base $\mathbf{C P}(\mathcal{H})$, as well as of its canonical kernel, are used as part of the arguments to prove such equivalences.

Apparently, the above physical interpretation and treatment of reproducing kernels has remained unknown for the pure mathematical line of 
research referred to in former lines. Indeed, we have been aware of the papers [24] and [25] only after having written the most substantial part of the present article. Since we consider here vector bundles with infinitedimensional fibers of infinite-dimensional manifolds, our work can be seen partly as a (non-trivial) extension to that setting of the categorial results of [25]. However, it must be noticed that, whereas one of the aims of [25] is to establish equivalence of categories thought of as equivalent ways to viewing states of matter, we are rather interested in pointing out the properties of (generalized) reproducing kernels in themselves, so we present them as objects of a category and look for their canonical description in terms of their Grassmannian universal representatives.

The general plan of the article is as follows. In Section 1 we give some elements for a categorial theory of notions and results related to the main items of papers [5], and [3]. Consequently in Section 2 subcategories of objects of a positive character are singled out, laying particular emphasis on the concept of reproducing $(-*)$-kernels. In this setting, completely positive mappings on Hilbert spaces appear as objects of a suitable category, instead of being considered (as usually in the literature) as morphisms. Section 3 collects significant results involving pull-backs of vector bundles and kernels, in the setting dealt with in the paper. Several items of previous sections are established in Section 4 for Grassmann manifolds, as a preparation for the main section of the article, namely Section 5. Here, we establish the universality theorems making (generalized) reproducing kernels $K$ associated with a given (like-Hermitian) vector bundle $\Pi: D \rightarrow Z$ to appear as pullbacks of canonical ones living on Grassmannians. The essential tool to do that is a mapping $\zeta_{K}: Z \rightarrow \operatorname{Gr}\left(\mathcal{H}^{K}\right)$ like that one of above, but this time taking values in all of the Grassmann manifold $\operatorname{Gr}\left(\mathcal{H}^{K}\right)$ formed by all closed subspaces of $\mathcal{H}^{K}$. Here $\mathcal{H}^{K}$ is the reproducing kernel Hilbert space defined by $K$. Such a mapping $\zeta_{K}$ is of course an extension of the corresponding mappings considered in [25], and indeed was suggested by an intermediate extension introduced in [22], in a finite-dimensional context. By mimicking names originated from physical considerations, we are tempted to call the mapping $\zeta_{K}$ the quantization map of the bundle $\Pi$, and every element $\zeta_{K}(s)$, $s \in Z$, a coherent state for $\Pi$.

Finally, in Section 6 we give some applications or examples. We find particularly interesting the fact that any completely positive mapping taking values in the algebra of bounded operators on a Hilbert space, in analogy with what happens for kernels, can be obtained as the pull-back of a canonical (and universal) completely positive map associated with the tautological bundle of a Grassmann manifold. 


\section{A categorial framework for like-Hermitian structures}

\subsection{Like-Hermitian structures}

We briefly review here the like-Hermitian vector bundles introduced in [3] in order to study geometric models for representations of Banach-Lie groups and $C^{*}$-algebras.

Definition 1.1 Let $Z$ be a real Banach manifold with an involutive diffeomorphism $z \mapsto z^{-*}, Z \rightarrow Z$, that is, $\left(z^{-*}\right)^{-*}=z$ for all $z \in Z$. A like-Hermitian structure on a smooth vector bundle $\Pi: D \rightarrow Z$ is a family $\left\{(\cdot \mid \cdot)_{z, z^{-*}}\right\}_{z \in Z}$ with the following properties:

(a) For every $z \in Z,(\cdot \mid \cdot)_{z, z^{-*}}: D_{z} \times D_{z^{-*}} \rightarrow \mathbb{C}$ is a sesquilinear strong duality pairing.

(b) For all $z \in Z, \xi \in D_{z}$, and $\eta \in D_{z^{-*}}$ we have $\overline{(\xi \mid \eta)}_{z, z^{-*}}=(\eta \mid \xi)_{z^{-*}, z}$.

(c) If $V$ is an arbitrary open subset of $Z$, and $\Psi_{V}: V \times \mathcal{E} \rightarrow \Pi^{-1}(V)$ and $\Psi_{V^{-*}}: V^{-*} \times \mathcal{E} \rightarrow \Pi^{-1}\left(V^{-*}\right)$ are trivializations (whose typical fiber is a complex Banach space $\mathcal{E}$ ) of the vector bundle $\Pi$ over $V$ and $V^{-*}\left(:=\left\{z^{-*} \mid z \in V\right\}\right)$, respectively, then the function $(z, x, y) \mapsto$ $\left(\Psi_{V}(z, x) \mid \Psi_{V^{-*}}\left(z^{-*}, y\right)\right)_{z, z^{-*}}, V \times \mathcal{E} \times \mathcal{E} \rightarrow \mathbb{C}$ is smooth.

Remark 1.2 Condition (a) in Definition 1.1 means that the functional $(\cdot \mid \cdot)_{z, z^{-*}}: D_{z} \times D_{z^{-*}} \rightarrow \mathbb{C}$ is continuous, is linear in the first variable and antilinear in the second variable, and both the mappings

$$
\xi \mapsto(\xi \mid \cdot)_{z, z^{-*}}, \quad D_{z} \rightarrow\left(\bar{D}_{z^{-*}}\right)^{*}, \quad \text { and } \quad \eta \mapsto(\cdot \mid \eta)_{z, z^{-*}}, \quad \bar{D}_{z^{-*}} \rightarrow D_{z}^{*},
$$

are (not necessarily isometric) isomorphisms of complex Banach spaces. Here we denote, for any complex Banach space $\mathcal{Z}$, by $\mathcal{Z}^{*}$ its dual (complex) Banach space and by $\overline{\mathcal{Z}}$ the complex-conjugate Banach space. That is, the real Banach spaces underlying $\mathcal{Z}$ and $\overline{\mathcal{Z}}$ coincide, and for any $z$ in the corresponding real Banach space and $\lambda \in \mathbb{C}$ we have $\lambda \cdot z$ (in $\overline{\mathcal{Z}})=\bar{\lambda} \cdot z$ (in $\mathcal{Z}$ ).

Remark 1.3 For later use we now record the following fact: Let $\mathcal{X}$ and $\mathcal{Y}$ be complex Banach spaces endowed with a sesquilinear strong duality pairing $(\cdot \mid \cdot): \mathcal{X} \times \mathcal{Y} \rightarrow \mathbb{C}$. If $\mathcal{H}$ is a complex Hilbert space and $T: \mathcal{H} \rightarrow \mathcal{X}$ is a bounded linear operator, then there exists a unique operator $S: \mathcal{Y} \rightarrow \mathcal{H}$ such that

$$
(\forall h \in \mathcal{H}, y \in \mathcal{Y}) \quad(T h \mid y)=(h \mid S y)_{\mathcal{H}}
$$

Conversely, for every bounded linear operator $S: \mathcal{Y} \rightarrow \mathcal{H}$ there exists a unique bounded linear operator $T: \mathcal{H} \rightarrow \mathcal{X}$ satisfying (1.1), and we denote $S^{-*}:=T$ and $T^{-*}:=S$. 
Like-Hermitian bundles admit a natural notion of morphism from one into another.

Definition 1.4 Let $\widetilde{\Pi}: \widetilde{D} \rightarrow \widetilde{Z}$ and $\Pi: D \rightarrow Z$ be like-Hermitian vector bundles, and assume that each of the manifolds $\widetilde{Z}$ and $Z$ is endowed with an involutive diffeomorphism denoted by $z \mapsto z^{-*}$ for both manifolds. A morphism (respectively, an antimorphism) of $\widetilde{\Pi}$ into $\Pi$ is a pair $\Theta=(\delta, \zeta)$ such that $\delta: \widetilde{D} \rightarrow D$ and $\zeta: \widetilde{Z} \rightarrow Z$ are smooth mappings satisfying the following conditions:

(i) The diagram

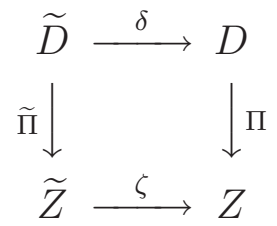

is commutative.

(ii) The mapping $\delta_{z}:=\left.\delta\right|_{\widetilde{D}_{z}}: \widetilde{D}_{z} \rightarrow D_{\zeta(z)}$ is a bounded linear operator whenever $z \in \widetilde{Z}$ (respectively, a bounded antilinear operator whenever $z \in \widetilde{Z}$ ).

(iii) For all $z \in \widetilde{Z}$ we have $\zeta\left(z^{-*}\right)=\zeta(z)^{-*}$.

Because of the assumption that the sesquilinear functionals $(\cdot \mid \cdot)_{z, z^{-*}}$ are strong duality pairings, it follows that the morphism $\Theta=(\delta, \zeta)$ is quasiadjointable in the sense that for every $z \in \widetilde{Z}$ there exists a (unique) bounded linear operator $\left(\delta_{z}\right)^{-*}: D_{\zeta(z)^{-*}} \rightarrow \widetilde{D}_{z^{-*}}$ such that

$$
\left(\forall \xi \in \widetilde{D}_{z}, \eta \in D_{\zeta(z)^{-*}}\right) \quad\left(\delta_{z} \xi \mid \eta\right)_{\zeta(z), \zeta(z)^{-*}}=\left(\xi \mid\left(\delta_{z}\right)^{-*} \eta\right)_{z, z^{-*}}
$$

If $\Theta=(\delta, \zeta)$ is an antimorphism, then it is again quasi-adjointable since for every $z \in \widetilde{Z}$ there exists a (unique) continuous antilinear operator $\left(\delta_{z}\right)^{-*}: D_{\zeta(z)^{-*}} \rightarrow \widetilde{D}_{z^{-*}}$ such that

$$
\left(\forall \xi \in \widetilde{D}_{z}, \eta \in D_{\zeta(z)^{-*}}\right) \quad\left(\delta_{z} \xi \mid \eta\right)_{\zeta(z), \zeta(z)^{-*}}={\overline{\left(\xi \mid\left(\delta_{z}\right)^{-*} \eta\right)_{z, z^{-*}}}}
$$

Now assume that $\zeta: \widetilde{Z} \rightarrow Z$ is a diffeomorphism. We say that the morphism $\Theta=(\delta, \zeta)$ is adjointable if there exists a morphism $\Theta^{-*}=\left(\delta^{-*}, \zeta^{-1}\right)$ from $\Pi$ into $\widetilde{\Pi}$ such that $(1.2)$ is satisfied with $\left(\delta_{z}\right)^{-*}:=\left.\delta^{-*}\right|_{D_{\zeta\left(z^{-*}\right)}}: D_{\zeta\left(z^{-*}\right)} \rightarrow \widetilde{D}_{z^{-*}}$. If this is the case, then any morphism $\Theta^{-*}$ with this property is said to be adjoint to $\Theta$. The adjointable antimorphisms are defined in a similar manner and an adjoint of an antimorphism is by definition an antimorphism. 
Definition 1.5 Let $\widetilde{\Pi}: \widetilde{D} \rightarrow \widetilde{Z}$ and $\Pi: D \rightarrow Z$ be two smooth like-Hermitian vector bundles. Assume that $\Theta=(\delta, \zeta)$ is a morphism from $\widetilde{\Pi}$ into $\Pi$. We say that $\Theta$ is an isometry if

$$
(\xi \mid \eta)_{z, z^{-*}}=(\delta(\xi) \mid \delta(\eta))_{\zeta(z), \zeta(z)^{-*}}
$$

whenever $z \in \widetilde{Z}, \xi \in \widetilde{D}_{z}$, and $\eta \in \widetilde{D}_{z^{-*}}$. Similarly, if $\Theta=(\delta, \zeta)$ is an antimorphism, then $\Theta$ is an isometry if and only if we have

$$
(\xi \mid \eta)_{z, z^{-*}}=\overline{(\delta(\xi) \mid \delta(\eta))}_{\zeta(z), \zeta(z)^{-*}}
$$

whenever $z \in \widetilde{Z}, \xi \in \widetilde{D}_{z}$, and $\eta \in \widetilde{D}_{z^{-*}}$.

Remark 1.6 In Definition 1.5, if $\Theta=(\delta, \zeta)$ is an isometry and the mapping $\delta_{z}:=\left.\delta\right|_{\widetilde{D}_{z}}: \widetilde{D}_{z} \rightarrow D_{\zeta(z)}$ is bijective for all $z \in \widetilde{Z}$, then $\Theta$ is quasi-adjointable and the appropriate condition (1.2) or (1.3) is satisfied if we take

$$
\left(\delta_{z}\right)^{-*}:=\left(\delta_{z^{-*}}\right)^{-1}: D_{\zeta(z)^{-*}} \rightarrow \widetilde{D}_{z^{-*}}
$$

for each $z \in \widetilde{Z}$. This also shows that if $\zeta: \widetilde{Z} \rightarrow Z$ is a diffeomorphism and $\Theta=(\delta, \zeta)$ is an isometric morphism (respectively, antimorphism) that is fiberwise bijective from $\widetilde{\Pi}$ to $\Pi$, then $\Theta$ is adjointable and its inverse $\Theta^{-1}=\left(\delta^{-1}, \zeta^{-1}\right)$ is an adjoint to $\Theta$.

It is plainly seen that like-Hermitian vector bundles, as the objects, and morphisms in between form a category which will be denoted here by LHer. There are several important examples of the above bundles which we think is worthwhile to present under the categorial language. In the next subsection we consider like-Hermitian vector bundles acted on by Banach-Lie groups.

\subsection{Group actions on vector bundles and representations}

Recall that an involutive Banach-Lie group is a (real or complex) Banach-Lie group $G$ equipped with a diffeomorphism $u \mapsto u^{*}$ satisfying $(u v)^{*}=v^{*} u^{*}$ and $\left(u^{*}\right)^{*}=u$ for all $u, v \in G$. In this case we denote

$$
u^{-*}:=\left(u^{-1}\right)^{*} \quad(\forall u \in G) \text { and } G^{+}:=\left\{u^{*} u \mid u \in G\right\},
$$

and the elements of $G^{+}$are called the positive elements of $G$. If $H$ is a Banach-Lie subgroup of $G$, then we say that $H$ is an involutive Banach-Lie subgroup if in addition $u^{*} \in H$ whenever $u \in H$. If $G$ is an involutive Banach-Lie group then for every $u \in G$ we have $\left(u^{-1}\right)^{*}=\left(u^{*}\right)^{-1}$ and moreover $\mathbf{1}^{*}=\mathbf{1}$. 
Definition 1.7 Assume that we have a complex involutive Banach-Lie group $G$, a holomorphic like-Hermitian vector bundle $\Pi: D \rightarrow Z$, and two holomorphic actions $\mu$ and $\nu$ of the group $G$ on $D$ and $Z$ respectively, such that

(i) there exists the commutative diagram

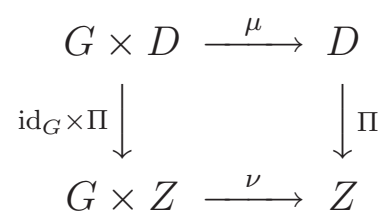

and for all $u \in G$ and $z \in Z$ the mapping $\left.\mu(u, \cdot)\right|_{D_{z}}: D_{z} \rightarrow D_{\nu(u, z)}$ is a bounded linear operator,

(ii) for every $u \in G$ and $z \in Z$ we have $\nu\left(u^{-*}, z^{-*}\right)=\nu(u, z)^{-*}$,

(iii) for every $z \in Z, u \in G ; \xi \in D_{z}, \eta \in D_{\nu\left(u^{-*}, z^{-*}\right)}$ we have

$$
\left(\mu\left(u^{*}, \eta\right) \mid \xi\right)_{z^{-*}, z}=(\eta \mid \mu(u, \xi))_{\nu\left(u^{-*}, z^{-*}\right), \nu(u, z)} .
$$

With the notation $(\Pi, G)$ we refer to a like-Hermitian vector bundle $\Pi$ which is acted on by the Banach-Lie group $G$ in the sense defined by the preceding properties.

Let $(\widetilde{\Pi}, \widetilde{G})$ and $(\Pi, G)$ be two such elements. We say that a pair $(\Theta, \alpha)$ is a morphism from $(\widetilde{\Pi}, \widetilde{G})$ into $(\Pi, G)$ if $\Theta:=(\delta, \zeta)$ is a morphism between the bundles $\widetilde{\Pi}$ and $\Pi, \alpha: \widetilde{G} \rightarrow G$ is a holomorphic group homomorphism which preserves involutions, and the following commutativity of diagrams holds:

(a) $\left(\operatorname{id}_{G} \times \Pi\right) \circ(\alpha \times \delta)=(\alpha \times \zeta) \circ\left(\operatorname{id}_{\widetilde{G}} \times \widetilde{\Pi}\right)$, that is,

$$
\begin{gathered}
\widetilde{G} \times \widetilde{D} \stackrel{\alpha \times \delta}{\longrightarrow} G \times D \\
\operatorname{id}_{\widetilde{G}} \times \widetilde{\Pi} \downarrow \\
\widetilde{G} \times \widetilde{Z} \stackrel{\alpha \times \zeta}{\longrightarrow} G \times Z
\end{gathered}
$$

(b) $\delta \circ \tilde{\mu}=\mu \circ(\alpha \times \delta)$ and $\zeta \circ \tilde{\nu}=\nu \circ(\alpha \times \zeta)$, that is,

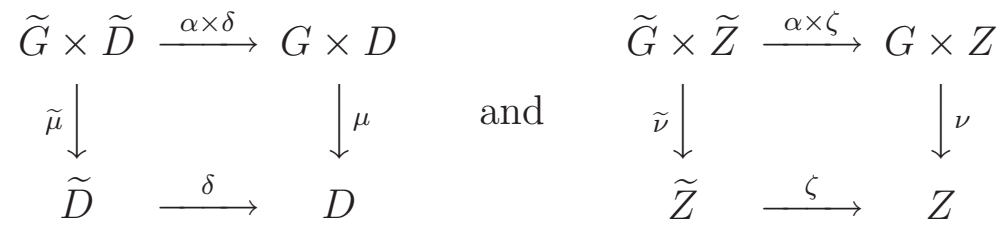

It is then straightforward to check that we have got a category where pairs $(\Pi, G)$ are the objects and pairs $(\Theta, \alpha)$ are the morphisms. Let GrLHer denote this category. 
Important examples of objects in GrLHer are suitably related to representations of groups acting in that category. Specifically, we shall make the following definition which claims its origins in the idea of basic mapping that shows up in Example 2.5 in [3].

Definition 1.8 In the setting of Definition 1.7, let us further assume that we have given a holomorphic $*$-representation $\pi: G \rightarrow \mathcal{B}(\mathcal{H})$.

We say that the mapping $\mathcal{R}: D \rightarrow \mathcal{H}$ relates $\Pi$ to $\pi$ if it has the following properties:

(i) $\mathcal{R}$ is holomorphic;

(ii) for each $z \in Z$ the mapping $\mathcal{R}_{z}:=\left.\mathcal{R}\right|_{D_{z}}: D_{z} \rightarrow \mathcal{H}$ is an injective bounded linear operator and we have $(\xi \mid \eta)_{z, z^{-*}}=(\mathcal{R}(\xi) \mid \mathcal{R}(\eta))_{\mathcal{H}}$ whenever $\xi \in D_{z}$ and $\eta \in D_{z^{-*}}$;

(iii) for every $u \in G$ and $z \in Z$ the diagram

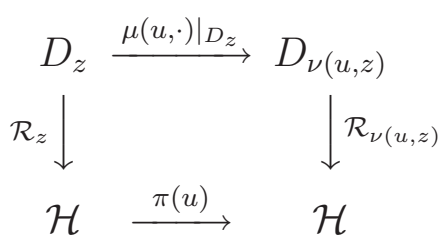

is commutative. We alternatively call $\mathcal{R}$ the transfer mapping between $\Pi$ and $\pi$ (or $D$ and $\mathcal{H}$ ).

In the case when an object $(\Pi, G)$ in the category GrLHer is associated with a representation $\pi$ and a transfer mapping $\mathcal{R}$ as in the above definition we shall refer to $(\Pi, G)$ by writing $(\Pi, G ; \pi)$. Given two of these objects $(\widetilde{\Pi}, \widetilde{G} ; \tilde{\pi}),(\Pi, G ; \pi)$ we say that a triple $(\Theta, \alpha, L)$ is a morphism from $(\widetilde{\Pi}, \widetilde{G} ; \tilde{\pi})$ into $(\Pi, G ; \pi)$ whenever $(\Theta, \alpha)$ is a morphism between $(\widetilde{\Pi}, \widetilde{G})$ and $(\Pi, G)$ in the sense of Definition 1.7, and $L: \widetilde{\mathcal{H}} \rightarrow \mathcal{H}$ is a bounded linear mapping such that:

(c) For all $u \in \widetilde{G}, \pi(\alpha(u)) \circ L=L \circ \tilde{\pi}(u)$.

(d) $\mathcal{R} \circ \delta=L \circ \widetilde{\mathcal{R}}$.

Then triples $(\Pi, G ; \pi)$ are the objects of a category, which we choose to call RepGLH, whose morphisms are the triples $(\Theta, \alpha, L)$. Of course properties (c) and (d) of $L$ are to be added to previous (a) and (b) for $(\Theta, \alpha)$.

There is an important subcategory of GrLHer (and of RepGLH) where the base spaces of the corresponding vector bundles are homogeneous manifolds. These bundles have been considered in [3], in connection with holomorphic realizations of representations. We recall their definition in the next subsection. 


\subsection{Homogeneous like-Hermitian vector bundles}

Assume that we have the following data:

- $G_{A}$ is an involutive real (respectively, complex) Banach-Lie group and $G_{B}$ is an involutive real (respectively, complex) Banach-Lie subgroup of $G_{A}$.

- For $X=A$ or $X=B$, assume $\mathcal{H}_{X}$ is a complex Hilbert space with $\mathcal{H}_{B}$ closed subspace in $\mathcal{H}_{A}$, and $\pi_{X}: G_{X} \rightarrow \mathcal{B}\left(\mathcal{H}_{X}\right)$ is a uniformly continuous (respectively, holomorphic) $*$-representation such that $\pi_{B}(u)=\left.\pi_{A}(u)\right|_{\mathcal{H}_{B}}$ for all $u \in G_{B}$. By $*$-representation we mean that $\pi_{A}\left(u^{*}\right)=\pi_{A}(u)^{*}$ for all $u \in G_{A}$.

- We denote by $P: \mathcal{H}_{A} \rightarrow \mathcal{H}_{B}$ the orthogonal projection.

Define an equivalence relation on $G_{A} \times \mathcal{H}_{B}$ by $(u, f) \sim\left(u^{\prime}, f^{\prime}\right)$ whenever there exists $w \in G_{B}$ such that $u^{\prime}=u w$ and $f^{\prime}=\pi_{B}\left(w^{-1}\right) f$. For every pair $(u, f) \in G_{A} \times \mathcal{H}_{B}$ its equivalence class is denoted by $[(u, f)]$ and the set of all equivalence classes is denoted by $D=G_{A} \times_{G_{B}} \mathcal{H}_{B}$. Clearly, there exists a natural onto map

$$
\Pi:[(u, f)] \mapsto s:=u G_{B}, \quad D \rightarrow G_{A} / G_{B} .
$$

For arbitrary $s \in G_{A} / G_{B}$, let $D_{s}:=\Pi^{-1}(s)$ denote the fiber over $s$. Note that $(u, f) \sim\left(u^{\prime}, f^{\prime}\right)$ implies that $\pi_{A}(u) f=\pi_{A}\left(u^{\prime}\right) f^{\prime}$ so that the correspondence $[(u, f)] \mapsto \pi_{A}(u) f, D_{s} \rightarrow \pi_{A}(u) \mathcal{H}_{B}$, gives rise to a complex linear structure on $D_{s}$, and a Hilbertian norm on $D_{s}$ defined by $\|[(u, f)]\|_{D_{s}}:=\left\|\pi_{A}(u) f\right\|_{\mathcal{H}_{A}}$ where $[(u, f)] \in D_{s}$. Moreover, the formula

$$
\begin{aligned}
\left([(u, f)] \mid\left[\left(u^{-*}, g\right)\right]\right)_{s, s^{-*}} & =\left(\pi_{A}(u) f \mid \pi_{A}\left(u^{-*}\right) g\right)_{\mathcal{H}_{A}} \\
& =\left(\pi_{A}\left(u^{-1}\right) \pi_{A}(u) f \mid g\right)_{\mathcal{H}_{A}} \\
& =(f \mid g)_{\mathcal{H}_{B}},
\end{aligned}
$$

for $[(u, f)] \in D_{s}$ and $\left[\left(u^{-*}, g\right)\right] \in D_{s^{-*}}$, defines a like-Hermitian structure $(\cdot \mid \cdot)_{s, s^{-*}}$ on the vector bundle $\Pi$. See more details in [3].

So, in the case where $G_{A}, G_{B}$ are complex and $\pi_{A}, \pi_{B}$ are holomorphic the bundle $\Pi$ is an example of Definition 1.7 with respect to the representation $\pi_{A}: G_{A} \rightarrow \mathcal{B}\left(\mathcal{H}_{A}\right)$ and the transfer mapping given by

$$
\mathcal{R}:[(u, f)] \mapsto \pi_{A}(u) f, \quad D \rightarrow \mathcal{H}_{A} .
$$

As in [3], we shall say that $\Pi: G_{A} \times_{G_{B}} \mathcal{H}_{B} \rightarrow G_{A} / G_{B}$ is the homogeneous like-Hermitian vector bundle associated with the data $\left(\pi_{A}, \pi_{B}, P\right)$. 
We can think of the data $\left(\pi_{A}, \pi_{B}, P\right)$ as objects of a category HomogLHer where the morphisms are just pairs $(\alpha, L)$ from $\left(\tilde{\pi}_{A}, \tilde{\pi}_{B}, \tilde{P}\right)$ into $\left(\pi_{A}, \pi_{B}, P\right)$ such that:

(i) $\alpha: \widetilde{G}_{A} \rightarrow G_{A}$ is a continuous (or holomorphic when $G_{A}$ and $G_{B}$ are complex) group homomorphism preserving the involutions, with the property $\alpha\left(\widetilde{G}_{B}\right) \subseteq G_{B}$.

(ii) $L: \widetilde{\mathcal{H}}_{A} \rightarrow \mathcal{H}_{A}$ is a bounded linear mapping such that $L \circ \tilde{\pi}_{A}(u)=$ $\pi_{A}(\alpha(u)) \circ L$ for every $u \in \widetilde{G}_{A}$ and $\left.P \circ L\right|_{\widetilde{\mathcal{H}}_{B}}=L \circ \widetilde{P}$ or, equivalently, $L\left(\widetilde{\mathcal{H}}_{B}\right) \subseteq \mathcal{H}_{B}$

We observe that the conditions on $(\alpha, L)$ allow us in particular to get the well-defined mapping

$$
[\alpha \times L]:[(u, f)] \mapsto[(\alpha(u), L(f))], \widetilde{G}_{A} \times_{\widetilde{G}_{B}} \widetilde{\mathcal{H}}_{B} \rightarrow G_{A} \times_{G_{B}} \mathcal{H}_{B} .
$$

The morphism from $\widetilde{G}_{A} \times_{\widetilde{G}_{B}} \widetilde{\mathcal{H}}_{B} \rightarrow \widetilde{G}_{A} / \widetilde{G}_{B}$ into $G_{A} \times_{G_{B}} \mathcal{H}_{B} \rightarrow G_{A} / G_{B}$ is given by the pair $\Theta=(\delta, \zeta)$, where $\delta=[\alpha \times L]$ and $\zeta=\alpha_{q}: u \widetilde{G}_{B} \mapsto \alpha(u) G_{B}$, $\widetilde{G}_{A} / \widetilde{G}_{B} \rightarrow G_{A} / G_{B}$. Moreover, it is an exercise of some patience to check that, for the transfer mapping defined just above and the natural multiplications, the morphism properties (a), (b), (c), (d) given in the definition of the category RepGLH hold for $\Theta=\left([\alpha \times L], \alpha_{q}\right)$.

In the following subsection we consider a remarkable class of objects in the category HomogLHer.

\subsection{Vector bundles arising from completely positive maps}

In this subsection we deal with completely positive maps on $C^{*}$-algebras valued in algebras of bounded operators on Hilbert spaces. Let us briefly review the Stinespring construction of dilations of completely positive maps. For details and references, see for instance [3].

For every complex vector space $X$ let $M_{n}(X)$ denote the space formed by all matrices $n \times n$ with entries in $X$. Then, for every linear map $\Phi: X \rightarrow Y$ between two vector spaces $X$ and $Y$ and every integer $n \geq 1$, put $\Phi_{n}=$ $\Phi \otimes \operatorname{id}_{M_{n}(\mathbb{C})}: M_{n}(X) \rightarrow M_{n}(Y)$, that is, $\Phi_{n}\left(\left(x_{i j}\right)_{1 \leq i, j \leq n}\right)=\left(\Phi\left(x_{i j}\right)\right)_{1 \leq i, j \leq n}$ for every matrix $\left(x_{i j}\right)_{1 \leq i, j \leq n} \in M_{n}(X)$.

Let $A$ be a unital $C^{*}$-algebra and let $\mathcal{H}_{0}$ be a complex Hilbert space. A linear map $\Phi: A \rightarrow \mathcal{B}\left(\mathcal{H}_{0}\right)$ is said to be a completely positive map if for every integer $n \geq 1$ the map $\Phi_{n}: M_{n}(A) \rightarrow M_{n}\left(\mathcal{B}\left(\mathcal{H}_{0}\right)\right)$ is positive in the sense that it takes positive elements in the $C^{*}$-algebra $M_{n}(A)$ to positive ones in $M_{n}\left(\mathcal{B}\left(\mathcal{H}_{0}\right)\right)$. If moreover $\Phi(\mathbf{1})=\mathbf{1}$ then we say that $\Phi$ is unital and in this case we have $\left\|\Phi_{n}\right\|=1$ for every $n \geq 1$. 
Define a nonnegative sesquilinear form on $A \otimes \mathcal{H}_{0}$ by the formula

$$
\left(\sum_{j=1}^{n} b_{j} \otimes \eta_{j} \mid \sum_{i=1}^{n} a_{i} \otimes \xi_{i}\right)=\sum_{i, j=1}^{n}\left(\Phi\left(a_{i}^{*} b_{j}\right) \eta_{j} \mid \xi_{i}\right)
$$

for $a_{1}, \ldots, a_{n}, b_{1}, \ldots, b_{n} \in A, \xi_{1}, \ldots, \xi_{n}, \eta_{1}, \ldots, \eta_{n} \in \mathcal{H}_{0}$ and $n \geq 1$. Set $N_{A}=\left\{x \in A \otimes \mathcal{H}_{0} \mid(x \mid x)=0\right\}$ and denote by $\mathcal{K}_{0}$ the Hilbert space obtained as the completion of $\left(A \otimes \mathcal{H}_{0}\right) / N_{A}$ with respect to the scalar product defined by $(\cdot \mid \cdot)$ on this quotient space. One can define a representation $\tilde{\pi}$ of $A$ by linear maps on $A \otimes \mathcal{H}_{0}$ given by

$$
(\forall a, b \in A)\left(\forall \eta \in \mathcal{H}_{0}\right) \quad \tilde{\pi}(a)(b \otimes \eta)=a b \otimes \eta .
$$

Then every linear map $\widetilde{\pi}(a): A \otimes \mathcal{H}_{0} \rightarrow A \otimes \mathcal{H}_{0}$ induces a continuous map $\left(A \otimes \mathcal{H}_{0}\right) / N_{A} \rightarrow\left(A \otimes \mathcal{H}_{0}\right) / N_{A}$, whose extension by continuity will be denoted by $\pi_{\Phi}(a) \in \mathcal{B}\left(\mathcal{K}_{0}\right)$. We thus obtain a unital $*$-representation $\pi_{\Phi}: A \rightarrow \mathcal{B}\left(\mathcal{K}_{0}\right)$ called the Stinespring representation associated with $\Phi$.

Additionally, denote by $V: \mathcal{H}_{0} \rightarrow \mathcal{K}_{0}$ the bounded linear map obtained as the composition

$$
V: \mathcal{H}_{0} \rightarrow A \otimes \mathcal{H}_{0} \rightarrow\left(A \otimes \mathcal{H}_{0}\right) / N_{A} \hookrightarrow \mathcal{K}_{0}
$$

where the first map is defined by $\mathcal{H}_{0} \ni h \mapsto \mathbf{1} \otimes h \in A \otimes \mathcal{H}_{0}$ and the second map is the natural quotient map. Then $V: \mathcal{H}_{0} \rightarrow \mathcal{K}_{0}$ is an isometry satisfying $\Phi(a)=V^{*} \pi_{\Phi}(a) V$ for all $a \in A$. In this sense $\pi_{\Phi}$ is called a Stinespring dilation of $\Phi$.

Note that if $\operatorname{dim} \mathcal{H}_{0}=1$, that is, $\Phi$ is a state of $A$, the Stinespring representation associated with $\Phi$ coincides with the corresponding GelfandNaimark-Segal (GNS) representation.

Particular cases of completely positive maps are the conditional expectations: Let $\mathbf{1} \in B \subseteq A$ be two $C^{*}$-algebras. A conditional expectation $E: A \rightarrow B$ is a linear map satisfying $E^{2}=E,\|E\|=1$ and $\operatorname{Ran} E=B$. Then it follows by the theorem of Tomiyama that $E(\mathbf{1})=\mathbf{1}$ and for every $a \in A, b_{1}, b_{2} \in B$ we have $E\left(a^{*}\right)=E(a)^{*}, 0 \leq E(a)^{*} E(a) \leq E\left(a^{*} a\right)$, and $E\left(b_{1} a b_{2}\right)=b_{1} E(a) b_{2}$. We have the following result (Lemma 6.7 in [3]).

Lemma 1.9 Let $B \subseteq A$ be two unital $C^{*}$-algebras with a conditional expectation $E: A \rightarrow B$ and a unital completely positive map $\Phi: A \rightarrow \mathcal{B}\left(\mathcal{H}_{0}\right)$ satisfying $\Phi \circ E=\Phi$, where $\mathcal{H}_{0}$ is a complex Hilbert space.

Now let $\pi_{A}: A \rightarrow \mathcal{B}\left(\mathcal{H}_{A}\right)$ and $\pi_{B}: B \rightarrow \mathcal{B}\left(\mathcal{H}_{B}\right)$ be the Stinespring representations associated with the unital completely positive maps $\Phi$ and $\left.\Phi\right|_{B}$, respectively. 
Then $\mathcal{H}_{B} \subseteq \mathcal{H}_{A}$, and for every $h_{0} \in \mathcal{H}_{0}$ and $b \in B$ we have the commutative diagrams

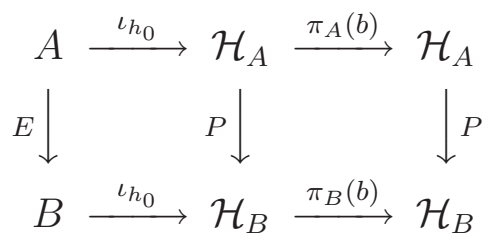

where $P: \mathcal{H}_{A} \rightarrow \mathcal{H}_{B}$ is the orthogonal projection, and $\iota_{h_{0}}: A \rightarrow \mathcal{H}_{A}$ is the map induced by $a \mapsto a \otimes h_{0}$.

Remark 1.10 The projection $P$ in Lemma 1.9 is indeed obtained from $E$ as the limit operator of expressions of the type $P\left(\left(\sum a_{i} \otimes y_{i}\right)+N_{A}\right):=$ $\sum\left(E\left(a_{i}\right) \otimes y_{i}\right)+N_{B}$, for every $\sum a_{i} \otimes y_{i} \in A \otimes \mathcal{H}_{A}$.

Let $\mathrm{G}_{X}$ be the group of invertible elements of $X$ for $X=A$ or $X=B$. Then $G_{X}$ has a natural structure of involutive complex Banach-Lie group defined by the involution of $X$. Moreover, $\mathrm{G}_{B}$ is a complex Banach-Lie subgroup of $\mathrm{G}_{A}$. We call the triple $\left(\left.\pi_{A}\right|_{\mathrm{G}_{A}},\left.\pi_{B}\right|_{\mathrm{G}_{B}}, P\right)$ the Stinespring data associated with $E$ and $\Phi$. It is clear that in this way we have obtained an object of the category HomogLHer. In fact, they form a subcategory of HomogLHer together with the morphisms defined as follows.

Let $\left(\pi_{\tilde{A}}, \pi_{\tilde{B}}, \tilde{P}\right)$ and $\left(\pi_{A}, \pi_{B}, P\right)$ be two Stinespring data. A morphism from $\left(\pi_{\tilde{A}}, \pi_{\tilde{B}}, \tilde{P}\right)$ into $\left(\pi_{A}, \pi_{B}, P\right)$ is any pair $(\alpha, T)$ such that $\alpha: \tilde{A} \rightarrow A$ is a unital $*$-homomorphism and $T: \tilde{\mathcal{H}}_{0} \rightarrow \mathcal{H}_{0}$ is a bounded linear mapping satisfying

(a) $\left.\alpha \circ \widetilde{E}\right|_{\widetilde{B}}=\left.E \circ \alpha\right|_{\widetilde{B}}$,

(b) $\Phi(\alpha(b)) \circ T=T \circ \widetilde{\Phi}(b)$ for every $b \in \tilde{B}$,

where the symbols involved in the above equalities have an obvious meaning. This category will be denoted as StLHer. We rewrite the objects $\left(\pi_{A}, \pi_{B}, P\right)$ as $(A, B, E ; \Phi)$.

To conclude this section, we remark that the categories introduced above are related through the following functors:

1. The functor GrLHer $\rightarrow$ LHer just given by the inclusion.

2. The functor HomogLHer $\rightarrow$ RepGLH defined by

$$
\left(\pi_{A}, \pi_{B}, P\right) \mapsto(\Pi, \pi ; \mathcal{R}) \quad \text { and } \quad(\alpha, L) \mapsto(\Theta, \alpha, L)
$$

where for $\pi_{A}: G_{A} \rightarrow \mathcal{B}\left(\mathcal{H}_{A}\right), \pi_{B}: G_{B} \rightarrow \mathcal{B}\left(\mathcal{H}_{B}\right)$, and $P: \mathcal{H}_{A} \rightarrow \mathcal{H}_{B}$, we take

$$
\begin{gathered}
\Pi:[(u, f)] \mapsto u \mathrm{G}_{B}, G_{A} \times_{\mathrm{G}_{B}} \mathcal{H}_{B} \rightarrow G_{A} / G_{B}, \\
\pi=\pi_{A}, \text { and } \mathcal{R}([(u, f)])=\pi_{A}(u) f,
\end{gathered}
$$

and for a morphism $(\alpha, L)$ in HomogLHer we take $\Theta=\left([\alpha \times L], \alpha_{q}\right)$. 
The functor HomogLHer $\rightarrow$ RepGLH entails a deep structural relationship between the corresponding categories, as it will be shown in Section 3, see Theorem 3.8.

In an analogue way to what has been done before, one could be tempted to affirm that the correspondence $(A, B, E ; \Phi) \mapsto\left(\pi_{A}, \pi_{B} ; P\right)$ defined by the Stinespring construction (described prior to Lemma 1.9) gives rise to a functor StLHer $\rightarrow$ HomogLHer, so that a morphism $(\alpha, T)$ in StLHer would be sent into a (possible) morphism in HomogLHer to be induced by the mapping

$$
\alpha \otimes T: \sum_{j} a_{j} \otimes h_{j} \mapsto \sum_{j} \alpha\left(a_{j}\right) \otimes T\left(h_{j}\right), \widetilde{A} \otimes \widetilde{\mathcal{H}}_{0} \rightarrow A \otimes \mathcal{H}_{0}
$$

Nevertheless, the extension of $\alpha \otimes T$ to $\mathcal{H}_{A}$ need not be well defined. An extra condition involving positivity on $(\alpha, T)$, which is sufficient for the purpose of such an extension and then for such a functor StLHer $\rightarrow$ HomogLHer, is proposed in the following section.

\section{Positivity. The category of reproducing $(-*)$-kernels}

There are elements making up the objects of the different categories of likeHermitian vector bundles which enjoy a neat character of positivity. So in the category RepGLH, where a transfer mapping $\mathcal{R}$ is positive (or positivedefinite) in the sense that for all $n \geq 1$ and every $s_{j} \in Z, \xi_{j} \in D_{s_{j}}$ for $j=1, \ldots, n$,

$$
\sum_{j, l=1}^{n}\left(\mathcal{R}\left(\xi_{j}\right) \mid \mathcal{R}\left(\xi_{l}\right)\right)_{\mathcal{H}}=\left(\sum_{j=1}^{n} \mathcal{R}\left(\xi_{j}\right) \mid \sum_{l=1}^{n} \mathcal{R}\left(\xi_{l}\right)\right) \geq 0
$$

Similarly, a $*$-representation $\pi_{A}$ taking part in an object of the category HomogLHer satisfies for all $n \geq 1$ and every $u_{j} \in G_{A}, h_{j} \in \mathcal{H}_{A}$ for $j=1, \ldots, n$,

$$
\sum_{j, l=1}^{n}\left(\pi_{A}\left(u_{j}\right) h_{j} \mid \pi_{A}\left(u_{l}\right) h_{l}\right)_{\mathcal{H}_{A}}=\left(\sum_{j=1}^{n} \pi_{A}\left(u_{j}\right) h_{j} \mid \sum_{l=1}^{n} \pi_{A}\left(u_{l}\right) h_{l}\right)_{\mathcal{H}_{A}} \geq 0 .
$$

Also, as regards the category StLHer, a (unital, in our case) completely positive mapping $\Phi: A \rightarrow \mathcal{B}\left(\mathcal{H}_{0}\right)$ satisfies by definition for all $n \geq 1$, every positive matrix $\left(a_{j, l}\right)_{j, l} \subset M_{n}(A)$ and $h_{1}, \ldots, h_{n} \in \mathcal{H}_{0}$,

$$
\sum_{j, l=1}^{n}\left(\Phi\left(a_{j, l}\right) h_{j} \mid h_{l}\right)_{\mathcal{H}_{0}} \geq 0
$$


As we are going to see below, the above self-imposed, by definition, properties are particular cases of a general notion of positivity linked to likeHermitian vector bundles.

Definition 2.1 Let $\Pi: D \rightarrow Z$ be a like-Hermitian bundle. A reproducing (-*)-kernel on $\Pi$ is a section $K \in \Gamma\left(Z \times Z\right.$, $\left.\operatorname{Hom}\left(p_{2}^{*} \Pi, p_{1}^{*} \Pi\right)\right)$ (whence $K(s, t): D_{t} \rightarrow D_{s}$ for all $\left.s, t \in Z\right)$ which is $(-*)$-positive definite in the following sense: For every $n \geq 1$ and $t_{j} \in Z, \eta_{j} \in D_{t_{j}^{-*}}(j=1, \ldots, n)$,

$$
\sum_{j, l=1}^{n}\left(K\left(t_{l}, t_{j}^{-*}\right) \eta_{j} \mid \eta_{l}\right)_{t_{l}, t_{l}^{-*}} \geq 0
$$

Here $p_{1}, p_{2}: Z \times Z \rightarrow Z$ are the natural projection mappings. If in addition $\Pi: D \rightarrow Z$ is a holomorphic like-Hermitian vector bundle, $K$ is continuous and $K(\cdot, s) \xi \in \mathcal{O}(Z, D)$ for all $\xi \in D_{s}$ and $s \in Z$, then we say that $K$ is a holomorphic reproducing (-*)-kernel. We shall say that a reproducing (-*)-kernel $K$ on a holomorphic like-Hermitian vector bundle $\Pi: D \rightarrow Z$ is holomorphic of the second kind if it is continuous and the mapping given by $\xi \mapsto K(t, \Pi(\xi)) \xi, D \rightarrow D$ is holomorphic for every $t \in Z$. Examples of kernels enjoying both types of holomorphy are the kernels $K^{\pi}$, defined through representations $\pi$, associated to the categories HomogLHer and sRep; see Remark 2.9(2) below.

Remark 2.2 Let $K(s, t)^{-*}: D_{s^{-*}} \rightarrow D_{t^{-*}}$ be the "adjoint" operator of the mapping $K(s, t)$ with respect to the family $(\cdot \mid \cdot)_{r, r^{-*}}, r \in Z$; i.e., $\left(K(s, t)^{-*} \xi^{-*} \mid \eta\right)_{t^{-*}, t}:=\left(\xi^{-*} \mid K(s, t) \eta\right)_{s^{-*}, s}$ for every $s, t \in Z$ and every $\xi^{-*} \in D_{s^{-*}}, \eta \in D_{t}$.

From the definition of reproducing kernel we have that $K\left(s, s^{-*}\right) \geq 0$ where we understand for this that $\left(K\left(s, s^{-*}\right) \xi \mid \xi\right)_{s, s^{-*}} \geq 0$ for all $\xi \in D_{s^{-*}}$. Moreover, by applying the (-*)-positiveness of $K$ to $s, t \in Z, \xi \in D_{s^{-*}}$ and $\eta \in D_{t^{-*}}$, we have

$$
\begin{aligned}
0 \leq & \left(K\left(s, s^{-*}\right) \xi \mid \xi\right)_{s, s^{-*}}+\left(K\left(s, t^{-*}\right) \eta \mid \xi\right)_{s, s^{-*}}+\left(K\left(t, s^{-*}\right) \xi \mid \eta\right)_{t, t^{-*}} \\
& +\left(K\left(t, t^{-*}\right) \eta \mid \eta\right)_{t, t^{-*}}
\end{aligned}
$$

whence we obtain that the sum $\left(K\left(s, t^{-*}\right) \eta \mid \xi\right)_{s, s^{-*}}+\left(K\left(t, s^{-*}\right) \xi \mid \eta\right)_{t, t^{-*}}$ is a real number. So

$$
\operatorname{Im}\left(K\left(s, t^{-*}\right) \eta \mid \xi\right)_{s, s^{-*}}=-\operatorname{Im}\left(K\left(t, s^{-*}\right) \xi \mid \eta\right)_{t, t^{-*}} .
$$

For $\xi \leftrightarrow i \xi$ we get $\operatorname{Im} i\left(K\left(s, t^{-*}\right) \eta \mid \xi\right)_{s, s^{-*}}=\operatorname{Im} i\left(K\left(t, s^{-*}\right) \xi \mid \eta\right)_{t, t^{-*}}$ hence

$$
\left(K\left(s, t^{-*}\right) \eta \mid \xi\right)_{s, s^{-*}}=\overline{\left(K\left(t, s^{-*}\right) \xi \mid \eta\right)_{t, t^{-*}}}=\left(\eta \mid K\left(s^{-*}, t\right) \xi\right)_{s, s^{-*}} .
$$

This implies that $K(a, b)^{-*}=K\left(b^{-*}, a^{-*}\right)$ for every $a, b \in Z$. 
Reproducing $(-*)$-kernels on like-Hermitian bundles have been introduced in [3] where they have been used to show that certain $*$-representations of involutive Banach-Lie groups (in particular Banach-Lie groups of invertibles in $C^{*}$-algebras) admit realizations on Hilbert spaces $\mathcal{H}^{K}$ of holomorphic sections on like-Hermitian vector bundles.

Such spaces $\mathcal{H}^{K}$ are constructed in the following way. For all $s \in Z$ and $\xi \in D_{s}$ denote $K_{\xi}=K(\cdot, s) \xi \in \Gamma(Z, D)$. (In fact $K_{\xi}$ is a holomorphic section, i.e., $K_{\xi} \in \mathcal{O}(Z, D)$, provided that the bundle $\Pi: D \rightarrow Z$ is holomorphic.) Then denote $\mathcal{H}_{0}^{K}:=\operatorname{span}\left\{K_{\xi} \mid \xi \in D\right\} \subseteq \Gamma(Z, D)$. The Hilbert space $\mathcal{H}^{K}$ is defined as the completion of $\mathcal{H}_{0}^{K}$ with respect to the scalar product given by

$$
\left(K_{\eta} \mid K_{\xi}\right)_{\mathcal{H}}=\left(K\left(s^{-*}, t\right) \eta \mid \xi\right)_{s^{-*}, s}
$$

whenever $s, t \in Z, \xi \in D_{s}$, and $\eta \in D_{t}$. See additional details in [3].

Each one of the categories GrLHer, RepGLH, HomogLHer, StLHer entails its own canonical type of $(-*)$-kernels. To begin with, let us consider the category GrLHer. In this case, for a bundle $\Pi$ being under the action of a group $G$, it sounds sensible, for a section $K$ to be a reproducing kernel on $\Pi$, to demand some condition relating $K$ and $G$. The suitability of the following one will emerge in Section 5 (Theorem 5.14).

Definition 2.3 Let $(\Pi, G)$ be an object in the category GrLHer. Let $K$ be a reproducing $(-*)$-kernel on $\Pi$. We say that $K$ is a reproducing $(-*)$-kernel on $(\Pi, G)$ if $K$ satisfies

$$
K(t, \nu(u, s)) \mu(u, \xi)=\mu\left(u, K\left(\nu\left(u^{-1}, t\right), s\right) \xi\right)
$$

for every $s, t \in Z, \xi \in D_{s}$, and $u \in G$.

We next describe the $(-*)$-kernels of the remaining categories in a categorial framework, showing in passing that the elements of positive character can be in turn regarded as objects of suitable categories.

Definition 2.4 Let $\widetilde{\Pi}: \widetilde{D} \rightarrow \widetilde{Z}$ and $\Pi: D \rightarrow Z$ be two like-Hermitian bundles, and let $\widetilde{K}$ and $K$ be reproducing $(-*)$-kernels on the bundles $\widetilde{\Pi}$ and $\Pi$, respectively. A morphism of reproducing $(-*)$-kernels from $\widetilde{K}$ to $K$ (respectively, an antimorphism of reproducing $(-*)$-kernels from $\widetilde{K}$ to $K$ ) is a morphism $\Theta=(\delta, \zeta)$ of $\widetilde{\Pi}$ into $\Pi$ (see Definition 1.4) (respectively, an antimorphism) with the property that there exists a constant $M>0$ such that for every integer $n \geq 1$, all $s_{1}, \ldots, s_{n} \in \widetilde{Z}$, and $\xi_{1} \in \widetilde{D}_{s_{1}}, \ldots, \xi_{n} \in \widetilde{D}_{s_{n}}$ 
we have

$$
\begin{aligned}
& \sum_{l, j=1}^{n}\left(K\left(\zeta\left(s_{l}\right)^{-*}, \zeta\left(s_{j}\right)\right) \delta\left(\xi_{j}\right) \mid\right.\left.\delta\left(\xi_{l}\right)\right)_{\zeta\left(s_{l}\right)^{-*}, \zeta\left(s_{l}\right)} \leq \\
& \leq M \sum_{l, j=1}^{n}\left(\widetilde{K}\left(s_{l}^{-*}, s_{j}\right) \xi_{j} \mid \xi_{l}\right)_{s_{l}^{-*}, s_{l}} .
\end{aligned}
$$

We denote by $\operatorname{Hom}(\widetilde{K}, K)$ the set of all morphisms from $\widetilde{K}$ to $K$.

It is clear that the composition of two morphisms of reproducing $(-*)$ kernels is again a morphism of reproducing $(-*)$-kernels. Consequently we get a category Kern whose objects are the reproducing (-*)-kernels on like-Hermitian bundles, and whose morphisms are defined as above.

Remark 2.5 In the setting of Definition 2.4 it is clear that the mapping

$$
\mathcal{H}_{0}^{\widetilde{K}} \rightarrow \mathcal{H}_{0}^{K}, \quad \sum_{j=1}^{n} \widetilde{K}_{\tilde{\xi}_{j}} \mapsto \sum_{j=1}^{n} K_{\delta\left(\tilde{\xi}_{j}\right)}
$$

is well defined and continuous, and it extends to a bounded linear operator $\mathcal{H}^{\Theta}: \mathcal{H}^{\widetilde{K}} \rightarrow \mathcal{H}^{K}$ (respectively, an antilinear operator if $\Theta$ is an antimorphism) satisfying $\left\|\mathcal{H}^{\Theta}\right\| \leq M$. (See Section 3 in [3].)

If $\Theta_{1}$ and $\Theta_{2}$ are morphisms or antimorphisms of reproducing $(-*)$ kernels whose composition $\Theta_{1} \circ \Theta_{2}$ makes sense, then we have

$$
\mathcal{H}^{\Theta_{1} \circ \Theta_{2}}=\mathcal{H}^{\Theta_{1}} \circ \mathcal{H}^{\Theta_{2}} .
$$

Definition 2.6 Let Hilb be the category whose objects are the Hilbert spaces over $\mathbb{C}$ and whose morphisms are the bounded linear operators. The reproducing kernel Hilbert space functor is the functor

$$
\mathcal{H}: \text { Kern } \rightarrow \text { Hilb }
$$

that takes every reproducing $(-*)$-kernel $K$ to the associated reproducing kernel Hilbert space $\mathcal{H}^{K}$ (see Definition 3.4 in [3]) and every morphism $\Theta \in \operatorname{Hom}(\widetilde{K}, K)$ to the bounded linear operator $\mathcal{H}^{\Theta}: \mathcal{H}^{\widetilde{K}} \rightarrow \mathcal{H}^{K}$. The fact that (2.6) is indeed a functor follows by (2.5).

Remark 2.7 The functor $\mathcal{H}$ was considered in [25] in the case of line vector bundles of finite dimension. Here $\mathcal{H}$ is also the main correspondence between categories that we consider in this paper, since it allows us to relate general kernels or fiber vector bundles with Grassmannian manifolds. In Proposition 2.8 below we establish other functors between the following categories: 
- Trans - its objects are the transfer mappings $\mathcal{R}$ associated with the objects of the category RepGLH, and where the morphisms are defined as the linear mappings $L$, appearing in the morphisms $(\Theta, \alpha, L)$ of RepGLH, for which there exists a constant $M>0$ such that for every integer $n \geq 1$, every $s_{1}, \ldots, s_{n} \in \widetilde{Z}$, and all $\xi_{1} \in \widetilde{D}_{s_{1}}, \ldots, \xi_{n} \in \widetilde{D}_{s_{n}}$ we have

$$
\sum_{l, j=1}^{n}\left(\mathcal{R}\left(L\left(\xi_{l}\right)\right) \mid \mathcal{R}\left(L\left(\xi_{j}\right)\right)\right)_{\mathcal{H}} \leq M \sum_{l, j=1}^{n}\left(\widetilde{\mathcal{R}}\left(\xi_{j}\right) \mid \widetilde{\mathcal{R}}\left(\xi_{l}\right)\right)_{\widetilde{\mathcal{H}}} .
$$

- sRep, whose objects are the *-representations associated with the objects of HomogLHer and the morphisms are the pairs $(\alpha, L)$, appearing as the morphisms of the category HomogLHer, for which there exists a constant $M>0$ such that for every integer $n \geq 1$, every $u_{1}, \ldots, u_{n} \in \widetilde{G}_{A}$, and all $h_{1}, \ldots, h_{n} \in \widetilde{\mathcal{H}}_{A}$ we have

$$
\begin{aligned}
& \sum_{l, j=1}^{n}\left(\pi_{A}\left(\alpha\left(u_{l}\right)\right)\left(L\left(h_{l}\right)\right) \mid\right.\left.\pi_{A}\left(\alpha\left(u_{j}\right)\right)\left(L\left(h_{j}\right)\right)\right)_{\mathcal{H}_{A}} \leq \\
& \leq M \sum_{l, j=1}^{n}\left(\tilde{\pi}_{A}\left(u_{l}\right) h_{l} \mid \tilde{\pi}_{A}\left(u_{j}\right) h_{j}\right)_{\tilde{\mathcal{H}}_{A}} .
\end{aligned}
$$

- CPos, whose objects are the completely positive maps associated with the objects of the category StLHer and the morphisms are the pairs $(\alpha, T)$ appearing in the morphisms of the category StLHer, for which there exists a constant $M>0$ such that for every integer $n \geq 1$, every positive $\left(a_{l j}\right) \subset M_{n}(\widetilde{A})$, and all $h_{1}, \ldots, h_{n} \in \widetilde{\mathcal{H}}_{0}$ we have

$$
\sum_{l, j=1}^{n}\left(\Phi\left(\alpha\left(a_{l, j}\right)\right) T\left(h_{l}\right) \mid T\left(h_{j}\right)\right)_{\mathcal{H}_{0}} \leq M \sum_{l, j=1}^{n}\left(\widetilde{\Phi}\left(a_{l j}\right) h_{l} \mid h_{j}\right)_{\widetilde{\mathcal{H}}_{0}} .
$$

Completely positive mappings have been considered as (relevant) morphisms of suitable categories of matrix ordered spaces or operator systems; see for instance [8], [12], [26], or [30]. It is maybe worth noticing that such mappings are viewed in the context developed here as objects of a category, rather than morphisms.

Proposition 2.8 The following correspondences define functors between the respective categories.

(i) The dilation functor $\boldsymbol{C P o s} \rightarrow \boldsymbol{s R e p}$ given by

$$
\Phi \mapsto \pi_{A} \quad \text { and } \quad(\alpha, T) \mapsto(\alpha, \alpha \otimes T),
$$


where $\pi_{A}$ is the Stinespring dilation associated with the completely positive mapping $\Phi$ and $\alpha \otimes T$ is the continuous extension to $\widetilde{\mathcal{H}}_{A}$ of the map defined in (1.5).

(ii) $\boldsymbol{s R e p} \rightarrow$ Trans given by

$$
\pi \mapsto \mathcal{R}^{\pi} \quad \text { and } \quad(\alpha, L) \mapsto(\Theta ; \alpha, L)
$$

where $\mathcal{R}^{\pi}([(u, f)])=\pi(u) f$, for every $u \in G_{A}, f \in \mathcal{H}_{B}$.

(iii) Trans $\rightarrow$ Kern given by

$$
\mathcal{R} \mapsto K^{\mathcal{R}} \quad \text { and } \quad(\Theta ; \alpha, L) \mapsto \Theta,
$$

where $K^{\mathcal{R}}(s, t)=\left(\mathcal{R}_{s^{-*}}\right)^{-*} \circ \mathcal{R}_{t}$, for every $s, t \in Z$. (Here, the expression $\left(\mathcal{R}_{s^{-*}}\right)^{-*}$ has the meaning explained in Remark 1.3.)

Proof. We shall show that the pair $(\alpha, \alpha \otimes T)$ is well defined as a morphism in the category sRep. The functorial property follows then readily.

So let $\widetilde{\Phi}: \widetilde{A} \rightarrow \mathcal{B}\left(\widetilde{\mathcal{H}}_{0}\right), \Phi: A \rightarrow \mathcal{B}\left(\mathcal{H}_{0}\right)$ objects in the category CPos, with associated triples $(\widetilde{A}, \widetilde{B}, \widetilde{E}),(A, B, E)$ respectively. Take a morphism $(\alpha, T): \widetilde{\Phi} \rightarrow \Phi$. This means that $\alpha: \widetilde{A} \rightarrow A$ is a unital algebra $*$-homomorphism with $\alpha(\widetilde{B}) \subseteq B$ and $T: \widetilde{\mathcal{H}}_{0} \rightarrow \mathcal{H}_{0}$ is a bounded linear operator. So

If $x=\sum_{i=1}^{n} a_{i} \otimes y_{i} \in N_{\widetilde{A}} \subseteq \widetilde{A} \otimes \widetilde{\mathcal{H}}_{0}$, then $\sum_{i, j=1}^{n}\left(\widetilde{\Phi}\left(a_{i}^{*} a_{j}\right) y_{j} \mid y_{i}\right)_{\widetilde{\mathcal{H}}_{0}}=0$,

$$
\sum_{i, j=1}^{n}\left(\Phi\left(\alpha\left(a_{i}\right)^{*} \alpha\left(a_{j}\right) T y_{j} \mid T y_{i}\right)_{\mathcal{H}_{0}} \leq M \sum_{i, j=1}^{n}\left(\widetilde{\Phi}\left(a_{i}^{*} a_{j}\right) y_{j} \mid y_{i}\right)_{\widetilde{\mathcal{H}}_{0}}=0\right.
$$

so $(\alpha \otimes T)(x) \in N_{A}$. Thus the quotient map

$$
(\alpha \otimes T)_{q}: \widetilde{A} \otimes \widetilde{\mathcal{H}}_{0} / N_{\widetilde{A}} \rightarrow A \otimes \mathcal{H}_{0} / N_{A}
$$

is well defined. Moreover, for $x \in \widetilde{A} \otimes \widetilde{\mathcal{H}}_{0}$ as above, we have

$$
\begin{aligned}
\|\alpha \otimes T(x)\|_{\mathcal{H}_{A}}^{2} & =\left\|\sum_{i} \alpha\left(a_{i}\right) \otimes T y_{i}\right\|_{\mathcal{H}_{A}}^{2}=\sum_{i, j}\left(\Phi\left(\alpha\left(a_{i}^{*} a_{j}\right)\right) T y_{j} \mid T y_{i}\right)_{\mathcal{H}_{0}} \\
& \leq M \sum_{i, j}\left(\widetilde{\Phi}\left(a_{i}^{*} a_{j}\right) y_{j} \mid y_{i}\right)_{\widetilde{\mathcal{H}}_{0}}=M\|x\|_{\widetilde{\mathcal{H}}_{A}}^{2}
\end{aligned}
$$

so that $(\alpha \otimes T)_{q}$ extends by continuity from $\widetilde{\mathcal{H}}_{A}$ to $\mathcal{H}_{A}$. We keep the notation $\alpha \otimes T$ for such an extension. 
Note that, in an analogous way, $\alpha \otimes T$ also extends continuously from $\widetilde{\mathcal{H}}_{B}$ to $\mathcal{H}_{B}$. Let us now see that $(\alpha, \alpha \otimes T)$ is a morphism from $\left(\pi_{\widetilde{A}}, \pi_{\widetilde{B}}, \widetilde{P}\right)$ into $\left(\pi_{A}, \pi_{B}, P\right)$ in HomogLHer. Since $\left.\alpha \circ \widetilde{E}\right|_{\widetilde{B}}=E \circ \alpha_{\widetilde{B}}$ it follows that $\alpha\left(\mathrm{G}_{\widetilde{B}}\right) \subseteq \mathrm{G}_{B}$ and, also,

$$
\begin{aligned}
P \circ(\alpha \otimes T)\left(\sum_{i} b_{i} \otimes y_{i}+N_{\widetilde{B}}\right) & =P\left(\sum_{i} \alpha\left(b_{i}\right) \otimes T y_{i}+N_{\widetilde{B}}\right) \\
& =\sum_{i} E\left(\alpha\left(b_{i}\right)\right) \otimes T y_{i}+N_{\widetilde{B}} \\
& =\sum_{i} \alpha\left(\widetilde{E}\left(b_{i}\right)\right) \otimes T y_{i}+N_{\widetilde{B}} \\
& =(\alpha \otimes T) \widetilde{P}\left(\sum_{i} b_{i} \otimes y_{i}+N_{\widetilde{B}}\right) .
\end{aligned}
$$

for each $\sum_{i} b_{i} \otimes y_{i}+N_{\widetilde{B}} \in \widetilde{B} \otimes \widetilde{\mathcal{H}}_{0}$. By density it follows that

$$
P \circ(\alpha \otimes T)=(\alpha \otimes T) \widetilde{P} \quad \text { on } \widetilde{\mathcal{H}}_{B}
$$

Now for every $u \in \mathrm{G}_{\widetilde{A}}$ and $\sum_{i} a_{i} \otimes y_{i}+N_{\widetilde{A}} \in \widetilde{A} \otimes \widetilde{\mathcal{H}}_{0}$,

$$
\begin{aligned}
(\alpha \otimes T) \pi_{\widetilde{A}}(u)\left(\sum_{i} a_{i} \otimes y_{i}+N_{\widetilde{A}}\right) & =\sum_{i} \alpha\left(u a_{i}\right) \otimes T y_{i}+N_{\widetilde{A}} \\
& =\pi_{A}(\alpha(u))\left(\sum_{i} \alpha\left(a_{i}\right) \otimes T y_{i}+N_{\widetilde{A}}\right) \\
& =\pi_{A}(\alpha(u))(\alpha \otimes T)\left(\sum_{i} a_{i} \otimes y_{i}+N_{\widetilde{A}}\right) .
\end{aligned}
$$

Again by density we obtain that

$$
(\alpha \otimes T) \pi_{\widetilde{A}}(u)=\pi_{A}(\alpha(u))(\alpha \otimes T)
$$

for all $u \in \mathrm{G}_{\widetilde{A}}$. Note that in particular, from the above equality we deduce that the mapping

$$
(\alpha, \alpha \otimes T):[(u, f)] \mapsto[(\alpha(u),(\alpha \otimes T) f)], \mathrm{G}_{\widetilde{A}} \times_{\mathrm{G}_{\widetilde{B}}} \mathcal{H}_{\widetilde{B}} \rightarrow \mathrm{G}_{A} \times_{\mathrm{G}_{B}} \mathcal{H}_{B}
$$

is well defined.

It is clear that

$$
\alpha_{q} \circ\left(\mathbf{1}_{\mathrm{G}_{\tilde{A}}} \times \widetilde{\Pi}\right)=\left(\mathbf{1}_{\mathrm{G}_{A}} \times \Pi\right) \circ(\alpha, \alpha \otimes T),
$$

where $\alpha_{q}$ is the quotient mapping defined by $\alpha_{q}\left(u \mathrm{G}_{\widetilde{B}}\right)=\alpha(u) \mathrm{G}_{B}$ for $u \in \mathrm{G}_{\widetilde{A}}$. Moreover,

$$
\alpha_{q}\left(u \mathrm{G}_{\widetilde{B}}\right)^{-*}=\alpha_{q}\left(\left(u \mathrm{G}_{\widetilde{B}}\right)^{-*}\right) \quad \text { for all } u \in \mathrm{G}_{\widetilde{A}} .
$$


Since all the above mappings are clearly holomorphic, we have shown that $\left((\alpha, \alpha \otimes T), \alpha_{q}\right)$ is a morphism in the category HomogLHer, as required in a first step.

Finally, for $n \geq 1, u_{i} \in \mathrm{G}_{\widetilde{A}}, f_{i}=\sum_{k=1}^{m} a_{k}^{i} \otimes y_{k}^{i}$ with $a_{k}^{i} \in \widetilde{A}$ and $y_{k}^{i} \in \widetilde{\mathcal{H}}_{0}$ $(i=1, \ldots, n)$, we get

$$
\begin{aligned}
& \sum_{i, j=1}^{n}\left(\pi_{A}\left(\alpha\left(u_{i}\right)\right)(\alpha \otimes T)\left(f_{i}\right) \mid \pi_{A}\left(\alpha\left(u_{j}\right)\right)(\alpha \otimes T)\left(f_{j}\right)\right)_{\mathcal{H}_{A}} \\
& =\sum_{i, j=1}^{n} \sum_{k=1}^{m}\left(\pi_{A}\left(\alpha\left(u_{i}\right)\right)\left(\alpha\left(a_{k}^{i}\right) \otimes T y_{k}^{i}\right) \mid \pi_{A}\left(\alpha\left(u_{j}\right)\right)\left(\alpha\left(a_{k}^{j}\right) \otimes T y_{k}^{j}\right)\right)_{\mathcal{H}_{A}} \\
& =\sum_{k=1}^{m} \sum_{i, j=1}^{n}\left(\alpha\left(u_{i} a_{k}^{i}\right) \otimes T y_{k}^{i} \mid \alpha\left(u_{j} a_{k}^{j}\right) \otimes T y_{k}^{j}\right)_{\mathcal{H}_{A}} \\
& =\sum_{k=1}^{m} \sum_{i, j=1}^{n}\left(\Phi\left(\alpha\left(\left(u_{j} a_{k}^{j}\right)^{*} u_{i} a_{k}^{i}\right)\right) T y_{k}^{i} \mid T y_{k}^{j}\right)_{\mathcal{H}_{0}} \\
& \leq \sum_{k=1}^{m} M \sum_{i, j=1}^{n}\left(\widetilde{\Phi}\left(\left(u_{j} a_{k}^{j}\right)^{*} u_{i} a_{k}^{i}\right) T y_{k}^{i} \mid y_{k}^{j}\right)_{\widetilde{\mathcal{H}}_{0}} \\
& =M \sum_{i, j=1}^{n}\left(\pi_{\widetilde{A}}\left(u_{i}\right) f_{i} \mid \pi_{\widetilde{A}}\left(u_{j}\right) f_{j}\right)_{\mathcal{H}_{\tilde{A}}}
\end{aligned}
$$

where in the inequality we have used that $(\alpha, T)$ is a morphism between $\widetilde{\Phi}$ and $\Phi$.

For arbitrary $\left(f_{i}\right) \subseteq \mathcal{H}_{\widetilde{A}}$ it is enough to approximate each $f_{i}$ by elements in $\widetilde{A} \otimes \widetilde{\mathcal{H}}_{0} / N_{\widetilde{A}}$ and apply the continuity of $\alpha \otimes T$ to the just proved inequality, to obtain that $(\alpha, \alpha \otimes T)$ is a morphism in the category sRep.

(ii) This part is quite obvious.

(iii) The statement is a simple consequence of the fact that one has

$$
\left(K^{\mathcal{R}}(s, t) \eta \mid \xi\right)_{s, s^{-*}}=\left(\left(\mathcal{R}_{s^{-*}}\right)^{-*}\left(\mathcal{R}_{t} \eta\right) \mid \xi\right)_{s, s^{-*}}=\left(\mathcal{R}_{t} \eta \mid \mathcal{R}_{s^{-*}} \xi\right)_{\mathcal{H}}
$$

for every $s, t \in Z, \xi \in D_{s^{-*}}$ and $\eta \in D_{t}$.

Remark 2.9 (1) The kernel $K^{\mathcal{R}}$ defined in part (iii) of the above proposition is a reproducing kernel on $(\Pi, G)$ in RepGLH. In fact, take $s, t \in Z$, $\xi \in D_{s}$ and $u \in G$. For simplicity, we put just in this remark $u \cdot s:=\nu(u, s)$ 
and $u \cdot \xi:=\mu(u, \xi)$. Then, for every $\eta \in D_{t^{-*}}$,

$$
\begin{aligned}
\left(K^{\mathcal{R}}(t, u \cdot s)(u \cdot \xi) \mid \eta\right)_{t, t^{-*}} & =\left(\mathcal{R}_{u \cdot s}(u \cdot \xi) \mid \mathcal{R}_{t^{-*}} \eta\right)_{\mathcal{H}} \\
& =\left(\left(\pi(u) \mathcal{R}_{s}\right)(\xi) \mid \mathcal{R}_{t^{-*}} \eta\right)_{\mathcal{H}} \\
& =\left(\mathcal{R}_{s} \xi \mid \pi(u)^{*} \mathcal{R}_{t^{-*}} \eta\right)_{\mathcal{H}} \\
& =\left(\mathcal{R}_{s} \xi \mid \pi\left(u^{*}\right) \mathcal{R}_{t^{-*}} \eta\right)_{\mathcal{H}} \\
& =\left(\mathcal{R}_{s} \xi \mid \mathcal{R}_{u^{*} \cdot t^{-*}}\left(u^{*} \cdot \eta\right)\right)_{\mathcal{H}} \\
& =\left(\left(\mathcal{R}_{u^{*} \cdot t^{-*}}\right)^{-*}\left(\mathcal{R}_{s} \xi\right) \mid u^{*} \cdot \eta\right)_{\left(u^{-1} \cdot t\right),\left(a^{*} \cdot t^{-*}\right)} \\
& =\left(u \cdot\left[\left(\mathcal{R}_{u^{*} \cdot t^{-*}}\right)^{-*}\left(\mathcal{R}_{s} \xi\right)\right] \mid \eta\right)_{t, t^{-*}} \\
& =\left(u \cdot\left[K^{\mathcal{R}}\left(u^{-1} \cdot t, s\right) \xi\right] \mid \eta\right)_{t, t^{-*}}
\end{aligned}
$$

where we have used (iii) of Definition 1.8 twice, and (ii) and (iii) of Definition 1.7. Since the sesquilinear form $(\cdot \mid \cdot)_{t, t^{-*}}$ is a strong duality pairing, by letting $\eta$ running over $D_{t^{-*}}$ one obtains $K^{\mathcal{R}}(t, u \cdot s)(u \cdot \xi)=u \cdot K^{\mathcal{R}}\left(u^{-1} \cdot t, s\right) \xi$ as we wanted to show.

It is worth noticing that, for any $(-*)$-kernel $K$ associated with a likeHermitian bundle $\Pi: D \rightarrow Z$, there always exists a Hilbert space $\mathcal{H}$ and a mapping $\mathcal{R}_{K}: D \rightarrow \mathcal{H}$ enjoying part of the properties of a transfer mapping, such that $K=K^{\mathcal{R}_{K}}$. In fact, one can choose $\mathcal{H}=\mathcal{H}^{K}$ as in the construction pointed out prior to Definition 2.3. Then by Proposition $3.7 \mathrm{c}$ ) of [3] there are the evaluation maps $\mathrm{ev}_{s}^{\iota}: \mathcal{H}^{K} \rightarrow D(s \in Z)$, so that $K(s, t)=\mathrm{ev}_{s}^{\iota} \circ\left(\mathrm{ev}_{t^{-*}}^{\iota}\right)^{-*}$ for all $s, t \in Z$. Thus one can define $\mathcal{R}_{K}(\xi):=\left(\mathrm{ev}_{s^{-*}}^{\iota}\right)^{-*}(\xi)$ for every $s \in Z$ and $\xi \in D_{s}$. It is routine to check that $K=K^{\mathcal{R}_{K}}$. However, one cannot assert that $\mathcal{R}_{K}$ is an isometry since $\left(\mathcal{R}_{K}(\xi) \mid \mathcal{R}_{K}(\eta)\right)_{\mathcal{H}^{K}}=(K(s, s) \xi \mid \eta)_{s, s^{-*}}$ for $s \in Z$ and $\xi \in D_{s}, \eta \in D_{s^{-*}}$.

(2) A kernel $K^{\pi}$ can be associated to a given representation $\pi \in \mathbf{s R e p}$ by the composition of functors sRep $\rightarrow$ Trans $\rightarrow$ Kern. Such a kernel is therefore given as $K^{\pi}(s, t)=\left(\mathcal{R}_{s^{-*}}\right)^{-*} \circ \mathcal{R}_{t}$ for $s, t \in D=G_{A} \times_{G_{B}} \mathcal{H}_{B}$, where $\mathcal{R}([(w, h)]):=\pi_{A}(w) h \in \mathcal{H}_{A}$ for every $[(w, h)] \in D$. Indeed, kernels of type $K^{\pi}$ admit alternatively a description as

$$
K^{\pi}(s, t) \eta=\left[\left(u, P\left(\pi_{A}\left(u^{-1}\right) \pi_{A}(v) f\right)\right)\right] \in D_{s}
$$

provided that $u, v \in G_{A}, s=u G_{B}, t=v G_{B}, \eta=[(v, f)] \in D_{t}$, and $P: \mathcal{H}_{A} \rightarrow \mathcal{H}_{B}$ is the orthogonal projection. In fact, for $\xi=\left[\left(u^{-*}, g\right)\right] \in D_{s^{-*}}$ we have

$$
\begin{aligned}
\left(K^{\pi}(s, t) \eta \mid \xi\right)_{s, s^{-*}} & =\left(\left(\mathcal{R}_{s^{-*}}\right)^{-*}\left(\mathcal{R}_{t} \eta\right) \mid \xi\right)_{s, s^{-*}}=\left(\mathcal{R}_{t} \eta \mid \mathcal{R}_{s^{-*}} \xi\right)_{\mathcal{H}_{A}} \\
& =\left(\pi_{A}(v) f \mid \pi_{A}\left(u^{-*}\right) g\right)_{\mathcal{H}_{A}}=\left(\pi_{A}\left(u^{-1}\right) \pi_{A}(v) f \mid g\right)_{\mathcal{H}_{B}} \\
& =\left(P\left(\pi_{A}\left(u^{-1}\right) \pi_{A}(v) f\right) \mid g\right)_{\mathcal{H}_{B}} \\
& =\left(\left[\left(u, P\left(\pi_{A}\left(u^{-1}\right) \pi_{A}(v) f\right)\right)\right] \mid\left[\left(u^{-*}, g\right)\right]\right)_{s, s^{-*}}
\end{aligned}
$$

where the last equality follows by (1.4). So (2.10) follows. 
In turn, the composition of functors CPos $\rightarrow$ sRep $\rightarrow$ Kern gives us what we call here the Stinespring kernel functor CPos $\rightarrow$ Kern defined by

$$
\Phi \mapsto K^{\Phi}, \quad(\alpha, T) \mapsto(\Theta, \alpha, L)
$$

where $K^{\Phi}$ is given as in (2.10), and where $\pi_{A}$ the Stinespring dilation of $\Phi$, $\Theta$ is the vector bundle morphism $\Theta=(\delta, \zeta)$ with $\delta=[\alpha \times L], \zeta=\alpha_{q}$, and $L=\alpha \otimes T$ as defined formerly.

The expression of $K^{\pi}$ given in (2.10) is the form under which the canonical kernels associated to homogeneous like-Hermitian bundles have been defined in [3], see also [5]. Such kernels are used in [3] to construct Hilbert spaces of holomorphic sections on those bundles.

\section{Operations on reproducing $(-*)$-kernels. Pull-backs}

In this section we discuss the operation of pull-back on reproducing kernels in the general setting of like-Hermitian bundles. A few special instances of this operation had been previously considered in the literature in the case of trivial bundles; see e.g., [23] or subsection 3.2 in [7]. The pull-backs will play a crucial role in connection with the universality theorems that we are going to establish in Section 5.

Definition 3.1 Let $\Pi: D \rightarrow Z$ be a like-Hermitian vector bundle, and denote by $p_{1}, p_{2}: Z \times Z \rightarrow Z$ the projection mappings. It is clear from Definition 3.1 in [3] that the set of all reproducing $(-*)$-kernels on $\Pi$ is closed under addition and under multiplication by positive scalars. Thus, that set is a convex cone in the complex vector space $\Gamma\left(Z \times Z, \operatorname{Hom}\left(p_{2}^{*} \Pi, p_{1}^{*} \Pi\right)\right)$; we shall denote this cone by $\mathcal{P} \mathcal{K}^{-*}(\Pi)$ and we shall call it the cone of reproducing $(-*)$-kernels on $\Pi$.

Definition 3.2 Let $\widetilde{\Pi}: \widetilde{D} \rightarrow \widetilde{Z}$ and $\Pi: D \rightarrow Z$ be like-Hermitian vector bundles, and assume that each of the manifolds $\widetilde{Z}$ and $Z$ is endowed with an involutive diffeomorphism denoted by $z \mapsto z^{-*}$ for both manifolds. Denote by $p_{1}, p_{2}: Z \times Z \rightarrow Z$ and $\widetilde{p}_{1}, \widetilde{p}_{2}: \widetilde{Z} \times \widetilde{Z} \rightarrow \widetilde{Z}$ the natural projections. Assume that $\Theta=(\delta, \zeta)$ is a morphism (or antimorphism) of $\widetilde{\Pi}$ into $\Pi$ (see Definition 1.4).

Now let $K \in \Gamma\left(Z \times Z, \operatorname{Hom}\left(p_{2}^{*} \Pi, p_{1}^{*} \Pi\right)\right)$ be a reproducing kernel on $\Pi$, where $p_{1}, p_{2}: Z \times Z \rightarrow Z$ are the natural projections. Then the pull-back of $K$ by $\Theta$ is the reproducing kernel

$$
\Theta^{*} K:=\widetilde{K} \in \Gamma\left(\widetilde{Z} \times \widetilde{Z}, \operatorname{Hom}\left(\widetilde{p}_{2}^{*} \widetilde{\Pi}, \widetilde{p}_{1}^{*} \widetilde{\Pi}\right)\right)
$$


defined by

$$
\widetilde{K}(s, t)=\left(\delta_{s^{-*}}\right)^{-*} \circ K(\zeta(s), \zeta(t)) \circ \delta_{t}
$$

for all $s, t \in Z$. Here $\left(\delta_{s^{-*}}\right)^{-*}: D_{\zeta(s)} \rightarrow \widetilde{D}_{s}$ is the operator defined by $(1.2)$ (or $(1.3)$ ).

It is easy to see that $\widetilde{K}$ is indeed a reproducing kernel on $\widetilde{\Pi}$. Note that formula (3.1) means that the following diagram

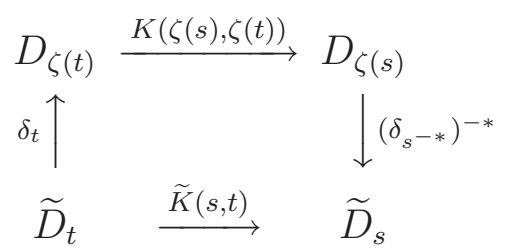

is commutative for all $s, t \in Z$.

Let us further observe that formula (3.1) gives us the equality in (2.4) of Definition 2.4, for $M=1$. In this sense we can interpret that the pullback of a given reproducing kernel $K$, with respect to a vector bundle $\Theta$, is another reproducing kernel $\Theta^{*} K$ for which $\Theta$ is extremal, say, for (2.4), or, alternatively, $M$-extremal for (2.4), with $M=1$.

Example 3.3 Let us consider the special case when both bundles $\underset{\widetilde{H}}{\widetilde{H}}$ and $\Pi$ are trivial and $\zeta$ is the identity map of some manifold $Z$. Thus, let $\widetilde{\mathcal{H}}$ and $\mathcal{H}$ be complex Hilbert spaces, and let $\kappa: Z \times Z \rightarrow \mathcal{B}(\mathcal{H})$ be a reproducing kernel in the classical sense (see e.g., [23]), which can be identified with a reproducing kernel $K$ on the trivial bundle $Z \times \mathcal{H} \rightarrow Z$ by $K(s, t)=$ $(s, t, \kappa(s, t))$ whenever $s, t \in Z$.

Next let $F: Z \rightarrow \mathcal{B}(\widetilde{\mathcal{H}}, \mathcal{H})$ be any mapping, which can be identified with a homomorphism $\delta$ from the trivial bundle $Z \times \widetilde{\mathcal{H}} \rightarrow Z$ into the trivial bundle $Z \times \mathcal{H} \rightarrow Z$ defined by $\delta(z, \widetilde{f})=(z, F(z) \widetilde{f})$ for all $z \in Z$ and $\widetilde{f} \in \widetilde{\mathcal{H}}$ (see for instance [21]). Now let $\Theta=\left(\delta, \mathrm{id}_{Z}\right)$ (compare Definition 3.2).

Then the pull-back of $K$ by $\Theta$ can be identified as above with a reproducing kernel (in the classical sense) defined by $(s, t) \mapsto F(s)^{*} \circ \kappa(s, t) \circ F(t)$, $Z \times Z \rightarrow \mathcal{B}(\widetilde{\mathcal{H}})$.

Now recall the setting of subsection 1.4. Let $A$ be a unital $C^{*}$-algebra and let $\mathcal{H}_{0}, \mathcal{H}_{A}$ be two complex Hilbert spaces. For a unital completely positive mapping $\Phi: A \rightarrow \mathcal{B}\left(\mathcal{H}_{0}\right)$, let $\pi_{A}: A \rightarrow \mathcal{B}\left(\mathcal{H}_{A}\right)$ be a Stinespring dilation of $\Phi$. Thus

$$
\Phi(a)=V^{*} \circ \pi_{A}(a) \circ V, \quad(a \in A)
$$


for some isometry $V: \mathcal{H}_{0} \rightarrow \mathcal{H}_{A}$. In the above example about trivial bundles, make the choice $Z=A, F(s)=V$ for all $s \in A$ so that $\delta=\operatorname{id}_{A} \times V$, and $\kappa(s, t)=\pi(s)$ for every $(s, t) \in A \times A$. Then the pull-back reproducing kernel of $\kappa$ by $\Theta:=\left(\delta, \operatorname{id}_{A}\right)$ is the mapping $\Phi:(s, t) \mapsto \Phi(s), A \times A \rightarrow \mathcal{B}\left(\mathcal{H}_{0}\right)$.

In other words, the fundamental relation (3.2) can be interpreted as a particular example of the pull-back operation for reproducing kernels.

Remark 3.4 If both $\widetilde{\Pi}$ and $\Pi$ are holomorphic bundles, the pair $(\delta, \zeta)$ is a homomorphism of $\widetilde{\Pi}$ into $\Pi$, the mapping $\zeta$ is holomorphic, and the mapping $\delta$ is anti-holomorphic, then the reproducing kernel $\Theta^{*} K$ is holomorphic provided $K$ is holomorphic.

Remark 3.5 In the setting of Definition 3.2, assume that $\Theta=(\delta, \zeta)$ is an isometry (see Definition 1.5) and in addition $\delta_{z}: \widetilde{D}_{z} \rightarrow D_{\zeta(z)}$ is a bijective map for all $z \in \widetilde{Z}$. In this case it follows by Remark 1.6 that for any reproducing kernel $K$ on $\Pi$ we have

$$
(\forall s, t \in \widetilde{Z}) \quad \Theta^{*} K(s, t)=\left(\delta_{s}\right)^{-1} \circ K(\zeta(s), \zeta(t)) \circ \delta_{t} .
$$

An important instance when the above situation occurs is when the bundle $\widetilde{\Pi}$ is the pull-back of $\Pi$ by $\zeta$, and $\delta$ is the associated map. (See [21] for details on pull-backs of vector bundles.)

The next result provides us in particular with a characterization of pullbacks of reproducing $-*$-kernels in terms of the corresponding reproducing kernel Hilbert spaces.

Proposition 3.6 Let $\widetilde{\Pi}: \widetilde{D} \rightarrow \widetilde{Z}$ and $\Pi: D \rightarrow Z$ be two like-Hermitian bundles, and let $\widetilde{K}$ and $K$ be reproducing $(-*)$-kernels on $\widetilde{\Pi}$ and $\Pi$, respectively. In addition, let $\Theta=(\delta, \zeta)$ be a morphism (respectively, antimorphism) of $\widetilde{\Pi}$ into $\Pi$. Then the following assertions hold:

(i) We have $\widetilde{K}=\Theta^{*} K$ if and only if $\Theta \in \operatorname{Hom}(\widetilde{K}, K)$ and the operator $\mathcal{H}^{\Theta}: \mathcal{H}^{\widetilde{K}} \rightarrow \mathcal{H}^{K}$ is an isometry.

(ii) Assume that $\Theta$ is an isometry of like-Hermitian structures and $\delta\left(\widetilde{D}_{\tilde{t}}\right)$ is dense in $D_{\zeta(\tilde{t})}$ for all $\tilde{t} \in \widetilde{Z}$. If $\widetilde{K}=\Theta^{*} K$ and both $\widetilde{K}$ and $K$ are reproducing $(-*)$-kernels, then the diagram

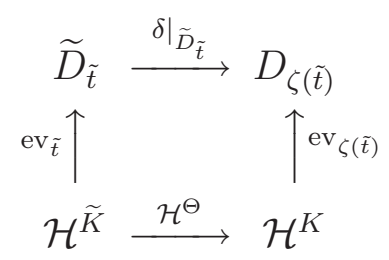

is commutative for all $\tilde{t} \in \widetilde{Z}$. 
Proof. (i) We have $\widetilde{K}=\Theta^{*} K$ if and only if for all $s, t \in \widetilde{Z}$,

$$
\widetilde{K}(s, t)=\left(\delta_{s^{-*}}\right)^{-*} \circ K(\zeta(s), \zeta(t)) \circ \delta_{t} .
$$

Now assume that $\Theta$ is a morphism. Since both $\widetilde{\Pi}$ and $\Pi$ are like-Hermitian bundles, condition (3.3) is further equivalent to the fact that for all $s, t \in \widetilde{Z}$, $\xi^{-*} \in \widetilde{D}_{s^{-*}}$, and $\eta \in \widetilde{D}_{t}$

$$
\left(\widetilde{K}(s, t) \eta \mid \xi^{-*}\right)_{s, s^{-*}}=\left(K(\zeta(s), \zeta(t)) \delta(\eta) \mid \delta\left(\xi^{-*}\right)\right)_{\zeta(s), \zeta(s)^{-*}},
$$

and this is equivalent to the fact that $\Theta \in \operatorname{Hom}(\widetilde{K}, K)$ and $\mathcal{H}^{\Theta}: \mathcal{H}^{\widetilde{K}} \rightarrow \mathcal{H}^{K}$ is an isometry. In fact, as noticed after Definition 3.2, in the setting of Definition 2.4 the latter condition means that in (2.4) we should always have equality and $M=1$.

On the other hand if $\Theta$ is an antimorphism, then condition (3.3) is equivalent to the fact that for all $\tilde{s}, \tilde{t} \in \widetilde{Z}, \tilde{\xi}^{-*} \in \widetilde{D}_{\tilde{s}^{-*}}$, and $\tilde{\eta} \in \widetilde{D}_{\tilde{t}}$,

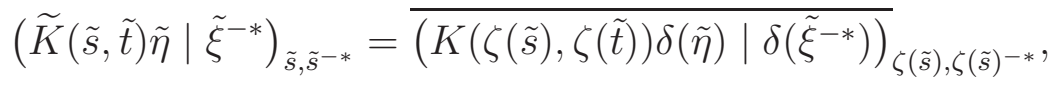

which is in turn equivalent to the fact that $\Theta \in \operatorname{Hom}(\widetilde{K}, K)$ and the operator $\mathcal{H}^{\Theta}: \mathcal{H}^{\widetilde{K}} \rightarrow \mathcal{H}^{K}$ is an isometry. (Note that both sides of (2.4) are real numbers hence are equal to their own complex-conjugates.)

(ii) Since $\operatorname{span}\left\{\widetilde{K}_{\tilde{\xi}} \mid \tilde{\xi} \in \widetilde{D}\right\}$ is dense in $\mathcal{H}^{\widetilde{K}}$, it will be enough to check that

$$
\left(\mathcal{H}^{\Theta}\left(\widetilde{K}_{\tilde{\xi}}\right)\right)(\zeta(\tilde{t}))=\delta\left(\widetilde{K}_{\tilde{\xi}}(\tilde{t})\right)
$$

Now let us assume that $\Theta$ is a morphism. We have $\left(\mathcal{H}^{\Theta}\left(\widetilde{K}_{\tilde{\xi}}\right)\right)(\zeta(\tilde{t}))=$ $K_{\delta(\tilde{\xi})}(\zeta(\tilde{t}))=K(\zeta(\tilde{t}), \zeta(\tilde{s})) \delta(\tilde{\xi})$, hence for arbitrary $\tilde{\eta}^{-*} \in \widetilde{D}_{\tilde{t}^{-*}}$ we get

$$
\begin{aligned}
\left(\left(\mathcal{H}^{\Theta}\left(\widetilde{K}_{\tilde{\xi}}\right)\right)(\zeta(\tilde{t})) \mid \delta\left(\tilde{\eta}^{-*}\right)\right)_{\zeta(\tilde{t}), \zeta(\tilde{t})^{-*}} & =\left(K(\zeta(\tilde{t}), \zeta(\tilde{s})) \delta(\tilde{\xi}) \mid \delta\left(\tilde{\eta}^{-*}\right)\right)_{\zeta(\tilde{t}), \zeta(\tilde{t})^{-*}} \\
& \stackrel{(3.4)}{=}\left(\widetilde{K}(\tilde{t}, \tilde{s}) \tilde{\xi} \mid \tilde{\eta}^{-*}\right)_{\tilde{t}_{,} \tilde{t}^{-*}} \\
& =\left(\widetilde{K}_{\tilde{\xi}}(\tilde{t}) \mid \tilde{\eta}^{-*}\right)_{\tilde{t}, \tilde{t}^{-*}} \\
& =\left(\delta\left(\widetilde{K}_{\tilde{\xi}}(\tilde{t})\right) \mid \delta\left(\tilde{\eta}^{-*}\right)\right)_{\zeta(\tilde{t}), \zeta(\tilde{t})^{-*}}
\end{aligned}
$$

where the latter equality follows by the hypothesis that $\Theta$ is an isometry of like-Hermitian structures (see Definition 1.5). Now we get equation (3.6) since $\left\{\delta\left(\tilde{\eta}^{-*}\right) \mid \tilde{\eta}^{-*} \in \widetilde{D}_{\tilde{t}^{-*}}\right\}$ is dense in $D_{\zeta(\tilde{t})^{-*}}$ by hypothesis, while $\Pi$ is a like-Hermitian bundle.

A similar reasoning works in the case when $\Theta$ is an antimorphism. 
The following statement is a version of Proposition III.3.3 in [23] in our framework. (See also the proof of Theorem 3 in [13].) It supplies conditions on a vector bundle $\Pi$ for an associated kernel $K$ to be the pullback of itself.

Corollary 3.7 Assume that $Z$ is a Banach manifold with an involutive diffeomorphism $Z \rightarrow Z, z \mapsto z^{-*}, \Pi: D \rightarrow Z$ is a like-Hermitian vector bundle, and $K$ is a reproducing $(-*)$-kernel on the bundle $\Pi$.

Now let $\tau: D \rightarrow D$ be a smooth map such that $\tau^{2}=\mathrm{id}_{D}$, and for all $z \in Z$ we have $\tau\left(D_{z}\right) \subseteq D_{z^{-*}},\left.\tau\right|_{D_{z}}: D_{z} \rightarrow D_{z^{-*}}$ is antilinear, and

$$
(\forall s \in Z)\left(\forall \xi \in D_{s}, \xi^{-*} \in D_{s^{-*}}\right) \quad\left(\tau(\xi) \mid \tau\left(\xi^{-*}\right)\right)_{s^{-*}, s}=\left(\xi^{-*} \mid \xi\right)_{s^{-*}, s} .
$$

Then we have

$$
(\forall s, t \in Z) \quad K(s, t)=\left.\tau^{-1} \circ K\left(s^{-*}, t^{-*}\right) \circ \tau\right|_{D_{t}},
$$

if and only if there exists an involutive antilinear isometry $\bar{\tau}: \mathcal{H}^{K} \rightarrow \mathcal{H}^{K}$ such that for all $\xi \in D$ we have $\bar{\tau}\left(K_{\xi}\right)=K_{\tau(\xi)}$. If this is the case, then

$$
\left(\forall F \in \mathcal{H}^{K}\right)(\forall t \in Z) \quad(\bar{\tau}(F))(t)=\tau\left(F\left(t^{-*}\right)\right) .
$$

Proof. First note that (3.8) is equivalent to the fact that $\Theta^{*} K=K$, where $\Theta$ is the antimorphism of the bundle $\Pi$ into itself defined by the pair of mappings $\tau$ and $z \mapsto z^{-*}$. Now the first assertion follows by Proposition 3.6(i) and Remark 2.5 with $\bar{\tau}:=\mathcal{H}^{\Theta}$.

To prove the second assertion we can use Proposition 3.6(ii).

The following theorem shows how objects of the category HomogLHer can be obtained as bundle pull-backs of objects of the category RepGLH. Further, this theorem points out the structural role of the transfer mappings $\mathcal{R}$ within these categories.

Theorem 3.8 Assume the setting of Definition 1.7. Let $z_{0} \in Z$ such that $z_{0}^{-*}=z_{0}$. Assume that the isotropy group $G_{0}:=\left\{u \in G \mid \nu\left(u, z_{0}\right)=z_{0}\right\}$ is a Banach-Lie subgroup of $G$ and in addition assume that the orbit $\mathcal{O}_{z_{0}}=$ $\left\{\nu\left(u, z_{0}\right) \mid u \in G\right\}$ of $z_{0}$ is a submanifold of $Z$, and denote by $i_{0}: \mathcal{O}_{z_{0}} \hookrightarrow Z$ the corresponding embedding map. Let $i_{0}^{*}(D)$ be the pull-back manifold of $\Pi: D \rightarrow Z$ through $i_{0}$, that is, $i_{0}^{*}(D):=\left\{(\xi, t) \in D \times \mathcal{O}_{z_{0}} \mid \Pi(\xi)=t\right\}$. Then there exists a closed subspace $\mathcal{H}_{0}$ of $\mathcal{H}$ such that the following assertions hold:

(i) For every $u \in G_{0}$ we have $\pi(u) \mathcal{H}_{0} \subseteq \mathcal{H}_{0}$.

(ii) Denote by $\pi: G_{0} \rightarrow \mathcal{B}\left(\mathcal{H}_{0}\right),\left.u \mapsto \pi(u)\right|_{\mathcal{H}_{0}}$, the corresponding representation of $G_{0}$ on $\mathcal{H}_{0}$, by $\Pi_{0}: D_{0} \rightarrow G / G_{0}$ the like-Hermitian vector bundle associated with the data $\left(\pi, \pi_{0}, P_{\mathcal{H}_{0}}\right)$, and by $\mathcal{R}_{0}: D_{0} \rightarrow \mathcal{H}$ the 
transfer mapping associated with the data $\left(\pi, \pi_{0}, P_{\mathcal{H}_{0}}\right)$ (as in subsection 1.3). Then there exists a biholmorphic bijective G-equivariant map $\theta: D_{0} \rightarrow i_{0}^{*}(D)$ such that $\theta$ sets up an isometric isomorphism of like-Hermitian vector bundles over $G / G_{0} \simeq \mathcal{O}_{z_{0}}$ and the diagram

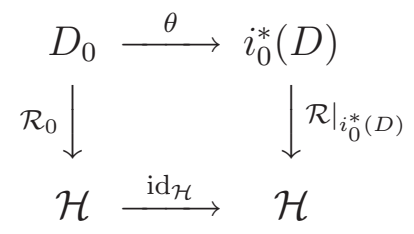

is commutative.

Proof. We shall take $\mathcal{H}_{0}:=\operatorname{Ran}\left(\mathcal{R}_{z_{0}}\right) \subseteq \mathcal{H}$

For arbitrary $u \in G_{0}$ we have $\nu\left(u, z_{0}\right)=z_{0}$. Then property (iii) in Definition 1.7 shows that we have a commutative diagram

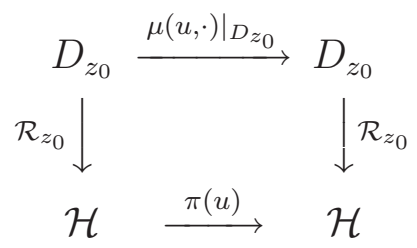

whence $\pi(u)\left(\mathcal{R}_{z_{0}}\left(D_{z_{0}}\right)\right) \subseteq \mathcal{R}_{z_{0}}\left(D_{z_{0}}\right)$, that is, $\pi(u) \mathcal{H}_{0} \subseteq \mathcal{H}_{0}$. Thus $\mathcal{H}_{0}$ has the desired property (i).

To prove (ii) we first note that, since $G_{0}$ is a Banach-Lie subgroup of $Z$, it follows that the $G$-orbit $\mathcal{O}_{z_{0}} \simeq G / G_{0}$ has a natural structure of Banach homogeneous space of $G$ (in the sense of [27]) such that the inclusion map $i_{0}: \mathcal{O}_{z_{0}} \hookrightarrow Z$ is an embedding.

Next define

$$
\begin{aligned}
\tilde{\theta}: G \times \mathcal{H}_{0} \rightarrow D, \quad \tilde{\theta}(u, f) & :=\mu\left(u, \mathcal{R}_{z_{0}}^{-1}(f)\right) \\
& =\Phi_{\nu\left(u, z_{0}\right)}^{-1}(\pi(u) f) \in D_{\nu\left(u, z_{0}\right)} \subseteq D,
\end{aligned}
$$

where the equality follows by property (iii) in Definition 1.7. Then for all $u \in G, u_{0} \in G_{0}$, and $f \in \mathcal{H}_{0}$ we have $\nu\left(u u_{0}^{-1}, z_{0}\right)=\nu\left(u, z_{0}\right)$ and

$$
\widetilde{\theta}\left(u u_{0}^{-1}, \pi\left(u_{0}\right) f\right)=\mathcal{R}_{\nu\left(u u_{0}^{-1}, z_{0}\right)}^{-1}\left(\pi\left(u u_{0}^{-1}\right) \pi\left(u_{0}\right) f\right)=\mathcal{R}_{\nu\left(u, z_{0}\right)}^{-1}(\pi(u) f)=\widetilde{\theta}(u, f) .
$$

In particular there exists a well defined map

$$
\theta: G \times_{G_{0}} \mathcal{H}_{0} \rightarrow D, \quad[(u, f)] \mapsto \mathcal{R}_{\nu\left(u, z_{0}\right)}^{-1}(\pi(u) f) .
$$

This mapping is $G$-equivariant with respect to the actions of $G$ on $G \times{ }_{G_{0}} \mathcal{H}$ and on $D$ since so is $\widetilde{\theta}$ : for all $u, v \in G$ and $f \in \mathcal{H}_{0}$ we have

$$
\begin{aligned}
\widetilde{\theta}(u v, f) & =\mathcal{R}_{\nu\left(u v, z_{0}\right)}^{-1}(\pi(u v) f)=\mathcal{R}_{\nu\left(u, \nu\left(v, z_{0}\right)\right)}^{-1}(\pi(u) \pi(v) f) \\
& =\mu\left(u, \mathcal{R}_{\nu\left(v, z_{0}\right)}^{-1}(\pi(v) f)\right)=\mu(u, \widetilde{\theta}(v, f)),
\end{aligned}
$$


where the second equality follows since $\nu: G \times Z \rightarrow Z$ is a group action, while the third equality follows by property (iii) in Definition 1.7. Besides, it is clear that $\theta$ is a bijection onto $i_{0}^{*}(D)$ and a fiberwise isomorphism. Also it is clear from the above construction of $\theta$ and from the definition of the transfer mapping $\mathcal{R}_{0}: D_{0} \rightarrow \mathcal{H}$ associated with the data $\left(\pi, \pi_{0}, P_{\mathcal{H}_{0}}\right)$ that $\mathcal{R} \circ \theta=\mathcal{R}_{0}$, that is, the diagram in the statement is indeed commutative. In addition, since both mappings $\mathcal{R}$ and $\mathcal{R}_{0}$ are fiberwise "isometric" (see property (ii) in Definition 1.7), it follows by $\mathcal{R} \circ \theta=\mathcal{R}_{0}$ that $\theta$ gives an isometric morphism of like-(-*)-Hermitian bundles over $G / G_{0} \simeq \mathcal{O}_{z_{0}}$.

Now we still have to prove that the map $\theta: D_{0}=G \times{ }_{G_{0}} \mathcal{H}_{0} \rightarrow i_{0}^{*}(D) \subseteq D$ is biholomorphic. We first show that it is holomorphic. Since $\mathcal{O}_{z_{0}}$ is a submanifold of $Z$, it follows that $i_{0}^{*}(D)$ is a submanifold of $D$ (see for instance the comments after Proposition 1.4 in Chapter III of [21]).

Thus it will be enough to show that $\theta: G \times{ }_{G_{0}} \mathcal{H}_{0} \rightarrow D$ is holomorphic. And this property is equivalent (by Corollary 8.3(ii) in [28]) to the fact that the mapping $\widetilde{\theta}: G \times \mathcal{H}_{0} \rightarrow D$ is holomorphic, since the natural projection $G \times \mathcal{H}_{0} \rightarrow G \times \times_{G_{0}} \mathcal{H}_{0}$ is a holomorphic submersion. Now the fact that $\widetilde{\theta}: G \times \mathcal{H}_{0} \rightarrow D$ is a holomorphic map follows by the first formula in its definition (3.9), since the group action $\alpha: G \times D \rightarrow D$ is holomorphic.

Consequently the mapping $\theta: G \times{ }_{G_{0}} \mathcal{H}_{0} \rightarrow i_{0}^{*}(D)$ is holomorphic. Then the fact that the inverse $\theta^{-1}: i_{0}^{*}(D) \rightarrow G \times{ }_{G_{0}} \mathcal{H}_{0}$ is also holomorphic follows by general arguments in view of the following facts (the first and the second of them have been already established, and the third one is well-known): Both $G \times_{G_{0}} \mathcal{H}_{0}$ and $i_{0}^{*}(D)$ are locally trivial holomorphic vector bundles; we have a commutative diagram

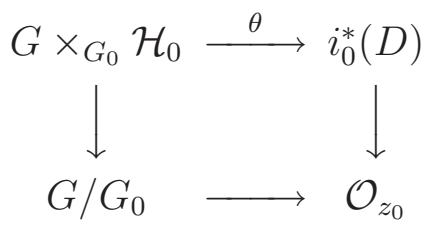

where the bottom arrow is the biholomorphic map $G / G_{0} \simeq \mathcal{O}_{z_{0}}$ induced by the action $\nu: G \times Z \rightarrow Z$, and the vertical arrows are the projections of the corresponding holomorphic like- $(-*)$-Hermitian vector bundles; the inversion mapping is holomorphic on the open set of invertible operators on a complex Hilbert space. Now the proof is finished.

The above result can be prolonged, to include morphisms of the respective categories, as follows. Let $(\widetilde{\Pi}, \widetilde{\pi} ; \widetilde{\mathcal{R}}),(\Pi, \pi ; \mathcal{R})$ be two objects in the category RepGLH, and let $(\Theta ; \alpha, L)$ be a morphism between them, with $\Theta=(\delta, \zeta)$. Suppose that both objects satisfy the assumptions of the previous theorem for $z_{0} \in \widetilde{Z}$ and $\zeta\left(z_{0}\right) \in Z$, with $\widetilde{G}_{0}, G_{0}$ and $\mathcal{O}_{z_{0}}, \mathcal{O}_{\zeta\left(z_{0}\right)}$ the isotropy subgroups and orbits, respectively. 
Let $\left(\tilde{\pi}, \tilde{\pi}_{0}, P_{\widetilde{\mathcal{H}}_{0}}\right),\left(\pi, \pi_{0}, P_{\mathcal{H}_{0}}\right)$ denote the data objects in HomogLHer constructed out of $(\widetilde{\Pi}, \tilde{\pi} ; \widetilde{\mathcal{R}}),(\Pi, \pi ; \mathcal{R})$, respectively, with bundle maps $(\tilde{\theta}, \tilde{\iota})$, $(\theta, \iota)$, as in part (ii) of Theorem 3.8. Put $\widetilde{P}_{0}=P_{\widetilde{\mathcal{H}}_{0}}, P_{0}=P_{\mathcal{H}_{0}}$.

Proposition 3.9 In the above setting, one has

$$
\alpha\left(\widetilde{G}_{0}\right) \subseteq G_{0} \quad \text { and } \quad L\left(\widetilde{\mathcal{H}}_{0}\right) \subseteq \mathcal{H}_{0} .
$$

Hence $\left(\alpha_{0}, L_{0}\right):=\left(\left.\alpha\right|_{\widetilde{G}_{0}},\left.L\right|_{\widetilde{\mathcal{H}}_{0}}\right)$ is a morphism between $\left(\tilde{\pi}, \tilde{\pi}_{0}, \widetilde{P}_{0}\right)$ and $\left(\pi, \pi_{0}, P_{0}\right)$. Moreover,

(1) $\theta \circ\left[\alpha_{0} \times L_{0}\right]=\delta \circ \tilde{\theta}$.

(2) $\Pi \circ\left[\alpha_{0} \times L_{0}\right]=\alpha_{q} \circ \widetilde{\Pi}_{0}$.

(3) $\iota \circ \alpha_{q}=\zeta \circ \tilde{\iota}$.

Proof. The inclusion $\alpha\left(\widetilde{G}_{0}\right) \subseteq G_{0}$ is equivalent to have $\nu\left(\alpha(u), \zeta\left(z_{0}\right)\right)=$ $\zeta\left(z_{0}\right)$ whenever $u \in \widetilde{G}$ satisfies $\tilde{\nu}\left(u, z_{0}\right)=z_{0}$, and this is an easy consequence of the fact that $\zeta \circ \tilde{\nu}=\nu \circ(\alpha \times \zeta)$, see assumption (b) prior to subsection 1.3. For the second inclusion recall that $\widetilde{\mathcal{H}}_{0}=\widetilde{\mathcal{R}}_{z_{0}}\left(\widetilde{D}_{z_{0}}\right)$ and $\mathcal{H}_{0}=\mathcal{R}_{\zeta\left(z_{0}\right)}\left(D_{\zeta\left(z_{0}\right)}\right)$ (note that $\zeta\left(z_{0}\right)=\zeta\left(z_{0}^{-*}\right)=\zeta\left(z_{0}\right)^{-*}$ ). By assumption (d) prior to subsection 1.3, we have $L_{0} \circ \widetilde{\mathcal{R}}_{z_{0}}=\left.\mathcal{R}_{\zeta\left(z_{0}\right)} \circ \delta\right|_{\widetilde{D}_{z_{0}}}$, so the inclusion $L\left(\widetilde{\mathcal{H}}_{0}\right) \subseteq \mathcal{H}_{0}$ holds.

Then we are going to prove (1), (2) and (3) of the statement.

(1) Take $[(u, f)] \in \widetilde{G} \times_{\widetilde{G}_{0}} \widetilde{\mathcal{H}}_{0}$. Then $\pi\left(\alpha_{0}(u)\right) L_{0}(f)=L \tilde{\pi}(u) f$ by (c) prior to subsection 1.3. Thus

$$
\begin{aligned}
\left(\theta \circ\left[\alpha_{0} \times L_{0}\right]\right)[(u, f)] & =\theta\left[\left(\alpha_{0}(u), L_{0}(f)\right)\right] \\
& =\mathcal{R}_{\nu\left(\alpha(u), \zeta\left(z_{0}\right)\right)}^{-1}\left(\pi(\alpha(u)) L_{0}(f)\right) \\
& =\mathcal{R}_{\nu\left(\alpha(u), \zeta\left(z_{0}\right)\right)}^{-1}(L \tilde{\pi}(u) f) \\
& =\mathcal{R}_{\nu\left(\alpha(u), \zeta\left(z_{0}\right)\right)}^{-1}\left(\left(L \circ \widetilde{\mathcal{R}}_{\tilde{\nu}\left(u, z_{0}\right)}\right)\left(\widetilde{\mathcal{R}}_{\tilde{\nu}\left(u, z_{0}\right)}^{-1} \tilde{\pi}(u) f\right)\right. \\
& =\mathcal{R}_{\nu\left(\alpha(u), \zeta\left(z_{0}\right)\right)}^{-1}\left(\mathcal{R}_{\zeta\left(\tilde{\nu}\left(u, z_{0}\right)\right)} \circ \delta\right)\left(\widetilde{\mathcal{R}}_{\tilde{\nu}\left(u, z_{0}\right)}^{-1} \tilde{\pi}(u) f\right) \\
& =\left(\delta \circ \widetilde{\mathcal{R}}_{\tilde{\nu}\left(u, z_{0}\right)}^{-1}\right)(\tilde{\pi}(u) f) \\
& =(\delta \circ \tilde{\theta})[(u, f)]
\end{aligned}
$$

where we have used (d) prior to subsection 1.3 in the fifth equality, and (b) prior to subsection 1.3 in the next-to-last equality.

(2) This is immediate.

(3) This is again a consequence of (b) prior to subsection 1.3. 
As it has been seen before, there are canonical reproducing $(-*)$-kernels $K^{\pi}$ and $K^{\mathcal{R}}$ associated with the vector bundles $\Pi_{0}: D_{0} \rightarrow \mathcal{O}_{z_{0}}, \Pi: D \rightarrow \mathcal{O}$, respectively, of Theorem 3.8. Recall that they are defined by

$$
K^{\mathcal{R}}(s, t):=\left(\mathcal{R}_{s^{-*}}\right)^{-*} \circ \mathcal{R}_{t} \quad \text { for } s, t \in Z
$$

and

$$
K^{\pi}(s, t):=\left(\mathcal{R}_{s^{-*}}^{0}\right)^{-*} \circ \mathcal{R}_{t}^{0}
$$

or, equivalently (according to Remark 2.9),

$$
K^{\pi}(s, t):=\left[\left(u, P_{0}\left(\pi(u)^{-1} \mathcal{R}^{0}(\cdot)\right)\right)\right]
$$

for every $s=u G_{0}, t=v G_{0} \in G / G_{0}$, where $\mathcal{R}^{0}$ is the transfer mapping associated with $\Pi_{0}$.

Corollary 3.10 The reproducing kernel $K^{\pi}$ is the pullback kernel of $K^{\mathcal{R}}$ corresponding to the bundle morphism $\Theta=(\theta, \iota)$ obtained in Theorem 3.8, that is,

$$
K^{\pi}(s, t)=\left(\theta_{s^{-*}}\right)^{-*} \circ K^{\mathcal{R}}(\iota(s), \iota(t)) \circ \theta_{t} \quad \text { for every } s, t \in \mathcal{O}_{z_{0}} \equiv G / G_{0}
$$

Proof. It has been noticed in the proof of Theorem 3.8 that $\mathcal{R} \circ \theta=\mathcal{R}^{0}$. Hence, for $s, t \in \mathcal{O}_{z_{0}} \subseteq Z$,

$$
\begin{aligned}
\left(\theta_{s^{-*}}\right)^{-*} \circ K^{\mathcal{R}}(\iota(s), \iota(t)) \circ \theta_{t} & =\left(\theta_{s^{-*}}\right)^{-*} \circ\left(\mathcal{R}_{\iota(s)-*}\right)^{-*} \circ \mathcal{R}_{\iota(s)} \circ \theta_{t} \\
& =\left(\mathcal{R}_{s^{-*}} \circ \theta_{s^{-*}}\right)^{-*} \circ\left(\mathcal{R}_{t} \circ \theta_{t}\right) \\
& =\left(\mathcal{R}_{s^{-*}}^{0}\right)^{-*} \circ \mathcal{R}_{t}^{0}
\end{aligned}
$$

as we wanted to show.

Remark 3.11 The significance of Theorem 3.8 is the following one: In the setting of Definition 1.7, the special situation of subsection 1.3 is met precisely when the action $\nu: G \times Z \rightarrow Z$ is transitive, and in this case the transfer mapping is essentially the unique mapping that relates the bundle $\Pi$ to the representation of the bigger group $G$.

On the other hand, by considering direct products of homogeneous Hermitian vector bundles, we can construct obvious examples of other maps relating bundles to representations as in Definition 1.7. Moreover, the canonical examples in this paper of both classes of vector bundles are related with the tautological ones linked to Grassmannians on Hilbert spaces. See next Section 4. 


\section{Grassmannians and universal reproducing kernels}

Let $\mathcal{H}$ be a complex Hilbert space and let $\mathcal{B}(\mathcal{H})$ be as above the $C^{*}$-algebra of bounded linear operators on $\mathcal{H}$ with the involution $T \mapsto T^{*}$ defined by the adjoint mapping. Let $\mathrm{GL}(\mathcal{H})$ be the Banach-Lie group of all invertible elements of $\mathcal{B}(\mathcal{H})$, and $\mathrm{U}(\mathcal{H})$ its Banach-Lie subgroup of all unitary operators on $\mathcal{H}$. For short, we put $\mathcal{G}=\operatorname{GL}(\mathcal{H})$ and $\mathcal{U}=\mathrm{U}(\mathcal{H})$. We recall the following notation from Example 4.6 in [4].

- $\operatorname{Gr}(\mathcal{H}):=\{\mathcal{S} \mid \mathcal{S}$ closed linear subspace of $\mathcal{H}\}$;

- $\mathcal{T}(\mathcal{H}):=\{(\mathcal{S}, x) \in \operatorname{Gr}(\mathcal{H}) \times \mathcal{H} \mid x \in \mathcal{S}\} \subseteq \operatorname{Gr}(\mathcal{H}) \times \mathcal{H}$

- $\Pi_{\mathcal{H}}:(\mathcal{S}, x) \mapsto \mathcal{S}, \mathcal{T}(\mathcal{H}) \rightarrow \operatorname{Gr}(\mathcal{H})$;

- for every $\mathcal{S} \in \operatorname{Gr}(\mathcal{H})$ we denote by $p_{\mathcal{S}}: \mathcal{H} \rightarrow \mathcal{S}$ the corresponding orthogonal projection.

These objects have the following well known properties:

(a) Both $\operatorname{Gr}(\mathcal{H})$ and $\mathcal{T}(\mathcal{H})$ have structures of complex Banach manifolds $(\operatorname{Gr}(\mathcal{H})$ is called the Grassmannian manifold of $\mathcal{H})$ and $\operatorname{Gr}(\mathcal{H})$ carries a natural (non-transitive) action of $\mathcal{U}$. (See Examples 3.11 and 6.20 in [28].) In fact, such an action extends to $\mathcal{G}$ as the inclusion $\mathcal{G} \hookrightarrow \mathcal{B}(\mathcal{H})$.

(b) The mapping $\Pi_{\mathcal{H}}: \mathcal{T}(\mathcal{H}) \rightarrow \operatorname{Gr}(\mathcal{H})$ is a holomorphic Hermitian vector bundle, and we call it the universal (tautological) vector bundle associated with the Hilbert space $\mathcal{H}$.

Definition 4.1 Denote by $p_{1}, p_{2}: \operatorname{Gr}(\mathcal{H}) \times \operatorname{Gr}(\mathcal{H}) \rightarrow \operatorname{Gr}(\mathcal{H})$ the natural projections and define

$$
Q_{\mathcal{H}}: \operatorname{Gr}(\mathcal{H}) \times \operatorname{Gr}(\mathcal{H}) \rightarrow \operatorname{Hom}\left(p_{2}^{*}\left(\Pi_{\mathcal{H}}\right), p_{1}^{*}\left(\Pi_{\mathcal{H}}\right)\right)
$$

by

$$
Q_{\mathcal{H}}\left(\mathcal{S}_{1}, \mathcal{S}_{2}\right)=\left.\left(p_{\mathcal{S}_{1}}\right)\right|_{\mathcal{S}_{2}}: \mathcal{S}_{2} \rightarrow \mathcal{S}_{1} \text { for all } \mathcal{S}_{1}, \mathcal{S}_{2} \in \operatorname{Gr}(\mathcal{H}) \text {. }
$$

The mapping $Q_{\mathcal{H}}$ is the universal reproducing kernel associated with the Hilbert space $\mathcal{H}$.

The vector bundle $\Pi_{\mathcal{H}}: \mathcal{T}(\mathcal{H}) \rightarrow \operatorname{Gr}(\mathcal{H})$ being Hermitian, it is in particular like-Hermitian for the involution on $\operatorname{Gr}(\mathcal{H})$ given by the identity map. Indeed, for the natural action of $\mathcal{G}$ on $\Pi_{\mathcal{H}}$, the natural representation $\mathcal{G} \rightarrow \mathcal{B}(\mathcal{H})$, and the involution $u \mapsto u^{-1}, \mathcal{G} \rightarrow \mathcal{G}$, one can associate the transfer mapping $\mathcal{R}_{\mathcal{H}}$ given by the projection in the second component $\mathcal{R}_{\mathcal{H}}:(S, x) \mapsto x, \mathcal{T}(\mathcal{H}) \rightarrow \mathcal{H}$. Thus the tautological bundle $\Pi_{\mathcal{H}}: \mathcal{T}(\mathcal{H}) \rightarrow \operatorname{Gr}(\mathcal{H})$, under the action of $\mathcal{G}=\mathrm{GL}(\mathcal{H})$, induces an object of the category RepGLH, and it is readily seen that the kernel $Q_{\mathcal{H}}$ is that one 
defined by the transfer mapping $\mathcal{R}_{\mathcal{H}}$. So the kernel $Q_{\mathcal{H}}$ defined above is an important example of object in the category Trans. We look for more examples of like-Hermitian structures in $\Pi_{\mathcal{H}}$ with non-trivial involutions in the base space $\operatorname{Gr}(\mathcal{H})$. For this purpose we introduce the following notion.

Definition 4.2 An involutive morphism (respectively, an involutive antimorphism) on the tautological vector bundle $\Pi_{\mathcal{H}}$ is a morphism (respectively, an antimorphism $) \Omega=(\gamma, \omega)$ from $\Pi_{\mathcal{H}}$ into itself such that the following conditions are satisfied:

(a) The map $\omega: \operatorname{Gr}(\mathcal{H}) \rightarrow \operatorname{Gr}(\mathcal{H})$ is an involutive diffeomorphism which we denote by $\mathcal{S} \mapsto \mathcal{S}^{-*}$.

(b) For every $\mathcal{S} \in \operatorname{Gr}(\mathcal{H})$ the mapping $\gamma_{\mathcal{S}}: \mathcal{S} \rightarrow \mathcal{S}^{-*}$ is an isometric linear (respectively, antilinear) bijective map and $\gamma_{\mathcal{S}^{-*}}=\left(\gamma_{\mathcal{S}}\right)^{-1}$.

(c) For all $\mathcal{S}_{1}, \mathcal{S}_{2} \in \operatorname{Gr}(\mathcal{H})$ and $x_{1} \in \mathcal{S}_{1}, x_{2} \in \mathcal{S}_{2}$ we have $\left(x_{1} \mid x_{2}\right)_{\mathcal{H}}=$ $\left(\gamma_{\mathcal{S}_{1}}\left(x_{1}\right) \mid \gamma_{\mathcal{S}_{2}}\left(x_{2}\right)\right)_{\mathcal{H}}$ (respectively, $\left.\left(x_{1} \mid x_{2}\right)_{\mathcal{H}}=\overline{\left(\gamma_{\mathcal{S}_{1}}\left(x_{1}\right) \mid \gamma_{\mathcal{S}_{2}}\left(x_{2}\right)\right)_{\mathcal{H}}}\right)$.

Now assume that $\Omega$ is an involutive morphism on the tautological vector bundle $\Pi_{\mathcal{H}}$. Then the like-Hermitian structure associated with $\Omega$ is the structure $\left\{(\cdot \mid \cdot)_{\mathcal{S}, \mathcal{S}^{-*}}\right\}_{\mathcal{S} \in \operatorname{Gr}(\mathcal{H})}$ on $\Pi_{\mathcal{H}}$ defined by

$$
(x \mid y)_{\mathcal{S}, \mathcal{S}^{-*}}=\left(x \mid \gamma_{\mathcal{S}^{-*}}(y)\right)_{\mathcal{H}}
$$

whenever $x \in \mathcal{S}, y \in \mathcal{S}^{-*}$, and $\mathcal{S} \in \operatorname{Gr}(\mathcal{H})$. It is easy to see that this is indeed a like-Hermitian structure. The vector bundle $\Pi_{\mathcal{H}}$ endowed with the like-Hermitian structure associated with $\Omega$ will be denoted by $\Pi_{\mathcal{H}, \Omega}$. The reproducing $(-*)$-kernel associated with $\Omega$ is the reproducing $(-*)$-kernel on $\Pi_{\mathcal{H}, \Omega}$,

$$
Q_{\mathcal{H}, \Omega}: \operatorname{Gr}(\mathcal{H}) \times \operatorname{Gr}(\mathcal{H}) \rightarrow \operatorname{Hom}\left(p_{2}^{*}\left(\Pi_{\mathcal{H}, \Omega}\right), p_{1}^{*}\left(\Pi_{\mathcal{H}, \Omega}\right)\right)
$$

defined by $Q_{\mathcal{H}, \Omega}\left(\mathcal{S}_{1}, \mathcal{S}_{2}\right)=p_{\mathcal{S}_{1}} \circ \gamma_{\mathcal{S}_{2}}: \mathcal{S}_{2} \rightarrow \mathcal{S}_{1}$ whenever $\mathcal{S}_{1}, \mathcal{S}_{2} \in \operatorname{Gr}(\mathcal{H})$.

Let us show that $Q_{\mathcal{H}, \Omega}$ is a reproducing $(-*)$-kernel on $\Pi_{\mathcal{H}, \Omega}$. For all $\mathcal{S}_{1}, \ldots, \mathcal{S}_{n} \in \operatorname{Gr}(\mathcal{H})$ and $x_{j} \in \mathcal{S}_{j}^{-*}$ for $j=1, \ldots, n$ we have

$$
\begin{aligned}
\sum_{j, l=1}^{n}\left(Q_{\mathcal{H}, \Omega}\left(\mathcal{S}_{l}, \mathcal{S}_{j}^{-*}\right) x_{j} \mid x_{l}\right)_{\mathcal{S}_{l}, \mathcal{S}_{l}^{-*}} & =\sum_{j, l=1}^{n}\left(p_{\mathcal{S}_{l}}\left(\gamma_{\mathcal{S}_{j}^{-*}}\left(x_{j}\right)\right) \mid x_{l}\right)_{\mathcal{S}_{l}, \mathcal{S}_{l}^{-*}} \\
& =\sum_{j, l=1}^{n}\left(p_{\mathcal{S}_{l}}\left(\gamma_{\mathcal{S}_{j}^{-*}}\left(x_{j}\right)\right) \mid \gamma_{\mathcal{S}_{l}^{-*}}\left(x_{l}\right)\right)_{\mathcal{H}} \\
& =\sum_{j, l=1}^{n}\left(\gamma_{\mathcal{S}_{j}^{-*}}\left(x_{j}\right) \mid \gamma_{\mathcal{S}_{l}^{-*}}\left(x_{l}\right)\right)_{\mathcal{H}} \\
& =\left(\sum_{j=1}^{n} \gamma_{\mathcal{S}_{j}^{-*}}\left(x_{j}\right) \mid \sum_{l=1}^{n} \gamma_{\mathcal{S}_{l}^{-*}}\left(x_{l}\right)\right)_{\mathcal{H}} \geq 0
\end{aligned}
$$


and

$$
\begin{aligned}
\left(x_{j} \mid Q_{\mathcal{H}, \Omega}\left(\mathcal{S}_{j}, \mathcal{S}_{l}^{-*}\right) x_{l}\right)_{\mathcal{S}_{j}^{-*}, \mathcal{S}_{j}} & =\left(x_{j} \mid p_{\mathcal{S}_{j}}\left(\gamma_{\mathcal{S}_{l}^{-*}}\left(x_{l}\right)\right)\right)_{\mathcal{S}_{l}, \mathcal{S}_{l}^{-*}} \\
& =\left(x_{j} \mid \gamma_{\mathcal{S}_{j}}\left(p_{\mathcal{S}_{j}}\left(\gamma_{\mathcal{S}_{l}^{-*}}\left(x_{l}\right)\right)\right)_{\mathcal{H}}\right. \\
& =\left(\gamma_{\mathcal{S}_{j}^{-*}}\left(x_{j}\right) \mid p_{\mathcal{S}_{j}}\left(\gamma_{\mathcal{S}_{l}^{-*}}\left(x_{l}\right)\right)\right)_{\mathcal{H}} \\
& =\left(\gamma_{\mathcal{S}_{j}^{-*}}\left(x_{j}\right) \mid \gamma_{\mathcal{S}_{l}^{-*}}\left(x_{l}\right)\right)_{\mathcal{H}} \\
& =\left(Q_{\mathcal{H}, \Omega}\left(\mathcal{S}_{l}, \mathcal{S}_{j}^{-*}\right) x_{j} \mid x_{l}\right)_{\mathcal{S}_{l}, \mathcal{S}_{l}^{-*}},
\end{aligned}
$$

where the latter equality was obtained during the previous calculation.

Hence we have that $Q_{\mathcal{H}, \Omega}$ is an object of the category Kern. We would like to have $Q_{\mathcal{H}, \Omega}$ in Trans but there is no reason for the existence of a transfer mapping, associated with the bundle $\Pi_{\mathcal{H}}$, from which $Q_{\mathcal{H}, \Omega}$ could emerge.

Remark 4.3 Every involutive isometric linear operator $C: \mathcal{H} \rightarrow \mathcal{H}$ defines an involutive morphism $\Omega_{C}=\left(\gamma_{C}, \omega_{C}\right)$ on $\Pi_{\mathcal{H}}$ by the formulas

$$
\omega_{C}: \mathcal{S} \mapsto C(\mathcal{S}), \quad \operatorname{Gr}(\mathcal{H}) \rightarrow \operatorname{Gr}(\mathcal{H}),
$$

and $\left(\gamma_{C}\right)_{\mathcal{S}}:=\left.C\right|_{\mathcal{S}}: \mathcal{S} \rightarrow C(\mathcal{S})$ whenever $\mathcal{S} \in \operatorname{Gr}(\mathcal{H})$. If $C: \mathcal{H} \rightarrow \mathcal{H}$ is an involutive isometric antilinear operator, then the same formulas define an involutive antimorphism $\Omega_{C}=\left(\gamma_{C}, \omega_{C}\right)$ on $\Pi_{\mathcal{H}}$.

We shall write $Q_{\mathcal{H}, C}$ to refer to $Q_{\mathcal{H}, \Omega}$ in the case when $\Omega=\Omega_{C}$. So we have

$$
Q_{\mathcal{H}, C}\left(S_{1}, S_{2}\right)=p_{S_{1}} \circ C: S_{2} \rightarrow S_{1} \quad \text { for } S_{1}, S_{2} \in \operatorname{Gr}(\mathcal{H}) .
$$

Similarly, we put $\Pi_{\mathcal{H}, C}=\Pi_{\mathcal{H}, \Omega_{C}}$.

Let us take a closer look at this example. Firstly, we notice that if we take the involution in $\mathcal{G}$ given by $u \mapsto C u^{-1} C, \mathcal{G} \rightarrow \mathcal{G}$, then the vector bundle $\Pi_{\mathcal{H}, C}$, satisfies, under the natural action of $\mathcal{G}$, those properties stated in Definition 1.7 which are independent of a (possible) transfer mapping. Furthermore, let $\mathcal{R}_{\mathcal{H}}^{C}$ denote the map $\mathcal{R}_{\mathcal{H}}:(S, x) \mapsto x, \mathcal{T}(\mathcal{H}) \rightarrow \mathcal{H}$ considered after Definition 4.1, where we assume that $\Pi_{\mathcal{H}}$ is endowed with the like-Hermitian pairings $(x \mid y)_{S, S^{-*}}:=(x \mid C y)_{\mathcal{H}}$, for every $S \in \operatorname{Gr}(\mathcal{H})$, $x \in S, y \in C(S)$. Then $\mathcal{R}_{\mathcal{H}, S}$ is the inclusion $\iota_{S}: S \hookrightarrow \mathcal{H}$, so that for every $y \in S^{-*}$ and $h \in \mathcal{H}$,

$$
\begin{aligned}
\left(\left(\mathcal{R}_{\mathcal{H}, S^{-*}}\right)^{-*} h \mid y\right)_{S, S^{-*}} & =\left(h \mid \mathcal{R}_{\mathcal{H}, S^{-*}} y\right)_{\mathcal{H}}=(h \mid y)_{\mathcal{H}}=(C h \mid C y)_{\mathcal{H}} \\
& =\left(p_{S} C(h) \mid C y\right)_{\mathcal{H}}=\left(p_{S} C(h) \mid C y\right)_{S, S^{-*}} .
\end{aligned}
$$

Hence $\left(\mathcal{R}_{\mathcal{H}, S^{-*}}\right)^{-*}=p_{S} \circ C$ whence we get $\left(\mathcal{R}_{\mathcal{H}, S_{1}}^{-*}\right)^{-*} \circ \mathcal{R}_{\mathcal{H}, S_{2}}=p_{S_{1}} \circ C \circ \iota_{S_{2}}=$ $Q_{\mathcal{H}, C}\left(S_{1}, S_{2}\right)$. 
Besides the above equality, properties (i) and (iii) in Definition 1.7 are clearly satisfied by $\mathcal{R}_{\mathcal{H}}^{C}$. Thus the map $\mathcal{R}_{\mathcal{H}}^{C}$ resembles a transfer mapping for $\Pi_{\mathcal{H}, C}$ as those of the category RepGLH. If $\mathcal{R}_{\mathcal{H}}^{C}$ were such a mapping then $Q_{\mathcal{H}, C}$ would become a kernel in the category Trans. But the mapping $\mathcal{R}_{\mathcal{H}}^{C}$ is not a transfer mapping because it does not satisfy property (ii) of Definition 1.7 .

By applying Theorem 3.8 to the bundle $\Pi_{\mathcal{H}}$ (with the identity map taken as involution in $\operatorname{Gr}(\mathcal{H})$ ) we obtain objects of the category HomogLHer in terms of Grassmannians. We next examine this example in detail.

Let $\mathcal{S}_{0} \in \operatorname{Gr}(\mathcal{H})$. The orbit of $\mathcal{S}_{0}$ in $\operatorname{Gr}(\mathcal{H})$ under the collineation action by $\mathcal{G}$ will be denoted by $\operatorname{Gr}_{\mathcal{S}_{0}}(\mathcal{H})$. It is well known that $\operatorname{Gr}_{\mathcal{S}_{0}}(\mathcal{H})$ coincides with the unitary orbit of $\mathcal{S}_{0}$ and with the connected component of $\mathcal{S}_{0}$ in $\operatorname{Gr}(\mathcal{H})$, and that it is given by

$$
\begin{aligned}
\operatorname{Gr}_{\mathcal{S}_{0}}(\mathcal{H}) & :=\left\{u \mathcal{S}_{0} \mid u \in \mathrm{GL}(\mathcal{H})\right\}=\left\{u \mathcal{S}_{0} \mid u \in \mathrm{U}(\mathcal{H})\right\} \\
& =\left\{\mathcal{S} \in \operatorname{Gr}(\mathcal{H}) \mid \operatorname{dim} \mathcal{S}=\operatorname{dim} \mathcal{S}_{0} \text { and } \operatorname{dim} \mathcal{S}^{\perp}=\operatorname{dim} \mathcal{S}_{0}^{\perp}\right\} \\
& \simeq \mathrm{U}(\mathcal{H}) /\left(\mathrm{U}\left(\mathcal{S}_{0}\right) \times \mathrm{U}\left(\mathcal{S}_{0}^{\perp}\right)\right) .
\end{aligned}
$$

(See Proposition 23.1 in [28] or Lemma 4.1 in [4].)

Let $\mathcal{G}\left(\left[p_{\mathcal{S}_{0}}\right]\right)$ denote the isotropy subgroup of $\mathcal{S}_{0}$; that is,

$$
\mathcal{G}\left(\left[p_{\mathcal{S}_{0}}\right]\right):=\left\{u \in \mathcal{G} \mid u \mathcal{S}_{0}=\mathcal{S}_{0}\right\} .
$$

This notation has been taken from [4] where it was suggested for reasons which can be found in that paper. Then

$$
\mathcal{G} / \mathcal{G}\left(\left[p_{\mathcal{S}_{0}}\right]\right) \simeq \operatorname{Gr}_{\mathcal{S}_{0}}(\mathcal{H})
$$

where the symbol " $\simeq$ " means diffeomorphism between the respective differentiable structures, and that the differentiable structure of the quotient space is the one associated with the quotient topology.

Set $\mathcal{T}_{\mathcal{S}_{0}}(\mathcal{H}):=\left\{(\mathcal{S}, x) \in \mathcal{T}(\mathcal{H}) \mid \mathcal{S} \in \operatorname{Gr}_{\mathcal{S}_{0}}(\mathcal{H})\right\}$. The vector bundle $\Pi_{\mathcal{H}, \mathcal{S}_{0}}: \mathcal{T}_{\mathcal{S}_{0}}(\mathcal{H}) \rightarrow \operatorname{Gr}_{\mathcal{S}_{0}}(\mathcal{H})$ obtained by restriction of $\Pi_{\mathcal{H}}$ to $\mathcal{T}_{\mathcal{S}_{0}}(\mathcal{H})$ will be called here the universal vector bundle at $\mathcal{S}_{0}$. It is also Hermitian and holomorphic. Moreover it is biholomorphically diffeomorphic to the vector bundle $\mathcal{G} \times_{\mathcal{G}([p])} \mathcal{S}_{0} \rightarrow \mathcal{G} / \mathcal{G}([p])$ where we write $p:=p_{\mathcal{S}_{0}}$ for brevity. Such a diffeomorphism is induced by the map defined between the total spaces of the respective bundles as $([u, x]) \mapsto\left(u \mathcal{S}_{0}, u x\right), \mathcal{G} \times{ }_{\mathcal{G}([p])} \mathcal{S}_{0} \rightarrow \mathcal{T}_{\mathcal{S}_{0}}$.

Then, by restriction to $\Pi_{\mathcal{H}, \mathcal{S}_{0}}$ of the pertinent elements previously considered in this section, we obtain that $\Pi_{\mathcal{H}, \mathcal{S}_{0}}$ defines an object of the category HomogLHer. So in particular the kernel

$$
Q_{\mathcal{H}, \mathcal{S}_{0}}\left(u_{1} \mathcal{S}_{0}, u_{2} \mathcal{S}_{0}\right)\left(u_{2} x\right):=u_{1} p_{\mathcal{S}_{0}} u_{1}^{-1} u_{2} x, \quad\left(u_{1}, u_{2} \in \mathcal{G}, x \in \mathcal{S}_{0}\right)
$$

is an object coming from the category sRep. 
As in the case of $\Pi_{\mathcal{H}}$ we wish to find now involutions in $\operatorname{Gr}_{\mathcal{S}_{0}}(\mathcal{H})$ different from the identity map. For this, one can take an involutive morphism satisfying an additional assumption. We illustrate this point by considering involutive isometries $C$ on $\mathcal{H}$.

Specifically, let $C: \mathcal{H} \rightarrow \mathcal{H}$ be a continuous involutive map which is either $\mathbb{C}$-linear or conjugate-linear, and let $\mathcal{S}_{0}$ be a closed linear subspace such that $C\left(\mathcal{S}_{0}\right)=\mathcal{S}_{0}$. It turns out that the orbit $\operatorname{Gr}_{\mathcal{S}_{0}}(\mathcal{H})$ is invariant under the involution $\mathcal{S} \mapsto C(\mathcal{S})$ of $\operatorname{Gr}(\mathcal{H})$, and then by restriction we will get an involution on $\operatorname{Gr}_{\mathcal{S}_{0}}(\mathcal{H})$. In fact,

$$
\operatorname{Gr}_{\mathcal{S}_{0}}(\mathcal{H})=\left\{D\left(\mathcal{S}_{0}\right) \mid D \in \mathcal{G}\right\}
$$

Now let $\mathcal{S} \in \operatorname{Gr}_{\mathcal{S}_{0}}(\mathcal{H})$ arbitrary. Then there exists $D \in \mathrm{G}(A)$ with $\mathcal{S}=$ $D\left(\mathcal{S}_{0}\right)$, so that $C(\mathcal{S})=C D\left(\mathcal{S}_{0}\right)=C D C^{-1}\left(\mathcal{S}_{0}\right)$ since $C\left(\mathcal{S}_{0}\right)=\mathcal{S}_{0}$. Since $C D C^{-1} \in \mathcal{G}$ irrespective of whether $C$ is $\mathbb{C}$-linear or conjugate-linear, it then follows by (4.1) that $C(\mathcal{S}) \in \operatorname{Gr}_{\mathcal{S}_{0}}(\mathcal{H})$. Thus the orbit $\operatorname{Gr}_{\mathcal{S}_{0}}(\mathcal{H})$ is indeed preserved by the involution $\mathcal{S} \mapsto C(\mathcal{S})$ of $\operatorname{Gr}(\mathcal{H})$. This property corresponds to the fact that the quotient manifold $\mathcal{G} / \mathcal{G}([p])$, which is diffeomorphic to $\operatorname{Gr}_{\mathcal{S}_{0}}(\mathcal{H})$, is invariant under the involution $u \mathcal{G}([p]) \mapsto u^{-*} \mathcal{G}([p])$ where for every $u \in \mathcal{G}$ we denote $u^{-*}:=C u C$.

Let us point out what is the form of the kernel $Q_{\mathcal{H}, C}$ restricted to the bundle $\Pi_{\mathcal{H}, \mathcal{S}_{0}}$. Suppose that $\mathcal{S} \in \operatorname{Gr}_{\mathcal{S}_{0}}(\mathcal{H})$. Then there exists $u \in \mathrm{U}(\mathcal{H})$ such that $u \mathcal{S}_{0}=\mathcal{S}$ and $u \mathcal{S}_{0}^{\perp}=\mathcal{S}^{\perp}$. Then $u p_{\mathcal{S}_{0}}=p_{\mathcal{S}} u$, that is, $p_{\mathcal{S}}=u p_{\mathcal{S}_{0}} u^{-1}$. Thus for all $u_{1}, u_{2} \in \mathrm{U}(\mathcal{H})$ and $x_{1}, x_{2} \in \mathcal{S}_{0}$ we have

$$
Q_{\mathcal{H}, C}\left(u_{1} \mathcal{S}_{0}, u_{2} \mathcal{S}_{0}\right)\left(C u_{2} x_{2}\right)=p_{u_{1} \mathcal{S}_{0}}\left(C u_{2} x_{2}\right)=u_{1} p_{\mathcal{S}_{0}}\left(u_{1}^{-1} C u_{2} x_{2}\right) \text {. }
$$

In short, $Q_{\mathcal{H}, C}\left(\mathcal{S}_{1}, \mathcal{S}_{2}\right)=Q_{\mathcal{H}}\left(\mathcal{S}_{1}, C\left(\mathcal{S}_{2}\right)\right) \circ C$.

The so chosen involution in $G r_{\mathcal{S}_{0}}(\mathcal{H})$ depends obviously on a pre-fixed involutive isometry $C$ on $\mathcal{H}$. Thus it seems to be too much having the pretension to obtain universality results associated with the manifold $G r_{\mathcal{S}_{0}}(\mathcal{H})$ in this situation. Searching for an alternative way to overcome this obstacle we are led to consider a (canonical) complexification of $G r_{\mathcal{S}_{0}}(\mathcal{H})$.

Let $B:=\{p\}^{\prime}$ be the commutant subalgebra of $p$ in $\mathcal{B}(\mathcal{H})$. The algebra $B$ is formed by the operators $T$ such that $T\left(\mathcal{S}_{0}\right) \subset \mathcal{S}_{0}, T\left(\mathcal{S}_{0}^{\perp}\right) \subset \mathcal{S}_{0}^{\perp}$. Moreover, $B$ is self-adjoint so that it is in fact a $C^{*}$-subalgebra of $\mathcal{B}(\mathcal{H})$. Let $\mathcal{G}(p)$ denote the group of invertibles in $B$. Clearly, $\mathcal{G}(p)$ is a (closed) BanachLie subgroup of $\mathcal{G}$, which moreover is stable under the operation of taking adjoints $u \mapsto u^{*}$.

Then, endowed with the quotient topology, the space $\mathcal{G} / \mathcal{G}(p)$ is a homogeneous Banach manifold enjoying the natural involution given by

$$
u \mathcal{G}(p) \mapsto u^{-*} \mathcal{G}(p), \mathcal{G} / \mathcal{G}(p) \rightarrow \mathcal{G} / \mathcal{G}(p)
$$

where $u^{-*}$ is this time the inverse of the adjoint operator of $u \in \mathcal{G}$. 
Further, the quotient manifold $\mathcal{G} / \mathcal{G}(p)$ can be described as the set of pairs $\left\{\left(u \mathcal{S}_{0}, u^{-*} \mathcal{S}_{0}\right): u \in \mathcal{G}\right\}$ so that the former involution takes the form

$$
\left(u \mathcal{S}_{0}, u^{-*} \mathcal{S}_{0}\right) \mapsto\left(u^{-*} \mathcal{S}_{0}, u \mathcal{S}_{0}\right) .
$$

Note that the fixed-point set of such an involution is

$$
\left\{\left(u \mathcal{S}_{0}, u \mathcal{S}_{0}\right) \mid u \in \mathcal{U}\right\} \equiv \operatorname{Gr}_{\mathcal{S}_{0}}(\mathcal{H})
$$

The preceding remarks tell us in particular that the orbit of projections $\mathcal{G} / \mathcal{G}(p) \simeq\left\{u p u^{-1}: u \in \mathcal{G}\right\}$ is a complexification of the (complex, in turn) manifold $\operatorname{Gr}_{\mathcal{S}_{0}}(\mathcal{H})$. This fact can be extended to the level of vector bundles, so that the bundle $\mathcal{G} \times_{\mathcal{G}(p)} \mathcal{S}_{0} \rightarrow \mathcal{G} / \mathcal{G}(p)$, which we shall denote by $\Pi_{\mathcal{H}}^{\mathrm{C}}$ in the sequel, becomes a complexification of the bundle $\mathcal{G} \times \mathcal{G}_{([p])} \mathcal{S}_{0} \rightarrow \operatorname{Gr}_{\mathcal{S}_{0}}(\mathcal{H})$, in a sense which is made precise in [3], Theorem 4.4. See [4] and [3] for details about the above results.

For $\mathcal{H}_{A}:=\mathcal{H}, \mathcal{H}_{B}:=\mathcal{S}_{0}, p:=p_{\mathcal{S}_{0}}$, the inclusion mapping $\pi_{A}: \mathcal{G} \hookrightarrow \mathcal{B}(\mathcal{H})$ and the restriction mapping $\pi_{B}:=\left.\pi_{A}\right|_{\mathcal{G}(p)}$, it is clear that the vector bundle $\Pi_{\mathcal{H}, \mathcal{S}_{0}}$ gives rise to the object $\left(\pi_{A}, \pi_{B} ; p\right)$ in (the notation for objects in) the category HomogLHer. In fact, $\left(\pi_{A}, \pi_{B} ; p\right)$ can be obtained as an object in the category StLHer. More precisely, for every closed subspace $\mathcal{K}$ of $\mathcal{S}_{0}$ there is a completely positive mapping $\Phi_{\mathcal{K}}$ on $\mathcal{B}(\mathcal{H})$ which by application of the Stinespring method (see subsection 1.4 ) yields the vector bundle $\Pi_{\mathcal{H}, \mathcal{S}_{0}}$.

Proposition 4.4 In the above setting, put

$$
E_{p}: T \mapsto p T p+(1-p) T(1-p), \mathcal{B}(\mathcal{H}) \rightarrow\{p\}^{\prime}
$$

Let $\mathcal{K}$ be a closed subspace of $\mathcal{S}_{0}$ and define the compression mapping

$$
\Phi_{\mathcal{K}}: T \mapsto p_{\mathcal{K}} \circ T \circ \iota_{\mathcal{K}}, \mathcal{B}(\mathcal{H}) \rightarrow \mathcal{B}(\mathcal{K}) .
$$

Then $\Phi_{\mathcal{K}}$ is a unital completely positive mapping with $\Phi_{\mathcal{K}} \circ E_{p}=\Phi_{\mathcal{K}}$ and such that the vector bundle defined by $\left(\mathcal{B}(\mathcal{H}),\{p\}^{\prime}, E_{p} ; \Phi_{\mathcal{K}}\right)$ coincides with $\Pi_{\mathcal{H}, \mathcal{S}_{0}}$.

Proof. The mapping $\Phi_{\mathcal{K}}$ of the statement is clearly unital. It is also completely positive: For an integer $n$, a positive matrix $\left(T_{i, j}\right)_{1 \leq i, j \leq n}$ in $M_{n}(\mathcal{B}(\mathcal{H}))$ and $x_{1}, \ldots, x_{n} \in \mathcal{K}$,

$$
\sum_{i, j=1}^{n}\left(\Phi_{\mathcal{K}}\left(T_{i, j}\right) x_{j} \mid x_{i}\right)_{\mathcal{K}}=\sum_{i, j=1}^{n}\left(p_{\mathcal{K}} T_{i, j} x_{j} \mid x_{i}\right)_{\mathcal{K}}=\sum_{i, j=1}^{n}\left(T_{i, j} x_{j} \mid x_{i}\right)_{\mathcal{H}} \geq 0
$$

since $\left(T_{i, j}\right)_{1 \leq i, j \leq n}$ is positive. 
For arbitrary $T \in \mathcal{B}(\mathcal{H})$ we get

$$
\Phi_{\mathcal{K}}\left(E_{p}(T)\right)=p_{\mathcal{K}} \circ E p(T) \circ \iota_{\mathcal{K}}=p_{\mathcal{K}}(p T p+(1-p) T(1-p)) \iota_{\mathcal{K}} .
$$

Since $\mathcal{K} \subseteq \mathcal{S}_{0}$ we have $(1-p) \iota_{\mathcal{K}}=0$, whence $\Phi_{\mathcal{K}}\left(E_{p}(T)\right)=\left(p_{\mathcal{K}} \circ p\right) \circ T \circ \iota_{\mathcal{K}}$. Also, for every $x, y \in \mathcal{K}$ we have $\left(p_{\mathcal{K}}(1-p) x \mid y\right)=\left((1-p) x \mid p_{\mathcal{K}} y\right)=0$ since $p_{\mathcal{K}} y \in \mathcal{K} \subseteq \mathcal{S}_{0}$ and $(1-p) x \in \mathcal{S}_{0}^{\perp}$. This means that $p_{\mathcal{K}} p=p_{\mathcal{K}}$. Thus $\Phi_{\mathcal{K}} E_{p}=\Phi_{\mathcal{K}}$.

Now we proceed with the Stinespring method. For every $b, a \in \mathcal{B}(\mathcal{H})$ and $x, y \in \mathcal{K}$ we have

$$
\left(\Phi_{\mathcal{K}}\left(a^{*} b\right) x \mid y\right)_{\mathcal{K}}=\left(p_{\mathcal{K}}\left(a^{*} b x\right) \mid y\right)_{\mathcal{K}}=\left(a^{*} b x \mid y\right)_{\mathcal{H}}=(b x \mid a y)_{\mathcal{H}} .
$$

From this, it follows that the norm $\|\cdot\|_{\Phi_{\mathcal{K}}}$ on $\mathcal{B}(\mathcal{H}) \otimes \mathcal{K}$ defined by the mapping $\Phi_{\mathcal{K}}$ as in subsection 1.4 is given by

$$
\left\|\sum_{j=1}^{n} b_{j} \otimes x_{j}\right\|_{\Phi_{\mathcal{K}}}^{2}=\left\|\sum_{j=1}^{n} b_{j} x_{j}\right\|^{2}
$$

for every $\sum_{j=1}^{n} b_{j} \otimes x_{j} \in \mathcal{B}(\mathcal{H}) \otimes \mathcal{K}$. Hence the associated null space $N$ is formed by all the above elements $\sum_{j=1}^{n} b_{j} \otimes x_{j} \in \mathcal{B}(\mathcal{H}) \otimes \mathcal{K}$ such that $\sum_{j=1}^{n} b_{j} x_{j}=0$. On the other hand, the map $\sum_{j=1}^{n} b_{j} \otimes x_{j} \mapsto \sum_{j=1}^{n} b_{j} x_{j}$, $\mathcal{B}(\mathcal{H}) \otimes \mathcal{K} \rightarrow \mathcal{H}$ is well defined (note that $\mathcal{H}$ is a left module on $\mathcal{B}(\mathcal{H})$ for the natural action) and surjective, and so we get that

$$
[\mathcal{B}(\mathcal{H}) \otimes \mathcal{K}] / N \simeq \mathcal{H}
$$

Moreover, it is straightforward to derive from the above that the Stinespring dilation obtained from $\Phi_{\mathcal{K}}$ is the identity mapping $\iota: \mathcal{B}(\mathcal{H}) \rightarrow \mathcal{B}(\mathcal{H})$.

In an analogue way one can show that $\left[\{p\}^{\prime} \otimes \mathcal{K}\right] / N_{0} \simeq \mathcal{S}_{0}$ where $N_{0}$ is the null space associated with the previous norm restricted to $\{p\}^{\prime} \otimes \mathcal{K}$. Here the only point to comment is perhaps the surjectivity of the mapping

$$
\sum_{j=1}^{n} b_{j} \otimes x_{j} \mapsto \sum_{j=1}^{n} b_{j} x_{j},\{p\}^{\prime} \otimes \mathcal{K} \rightarrow \mathcal{S}_{0}
$$

This can be proved for instance as follows. Given $x \in \mathcal{S}_{0}$ one can choose $T$ in $\mathcal{B}(\mathcal{H})$ and $k \in \mathcal{K}$ such that $T(k)=x$. Then $E_{p}(T) \in\{p\}^{\prime}$ and $E_{p}(T) k=x$ since $\mathcal{K} \subseteq \mathcal{S}_{0}$. Hence the above map is onto.

Putting all the above facts together the statement of the proposition follows readily. 


\section{The universality theorems}

We state and prove in this section the fundamental results which enable us to recover reproducing $(-*)$-kernels as pull-backs of the canonical kernels associated with Grassmannian tautological bundles. The first theorem shows up the existence of such pull-backs in purely algebraic terms.

Theorem 5.1 Let $\Pi: D \rightarrow Z$ be a like-Hermitian vector bundle and denote by $p_{1}, p_{2}: Z \times Z \rightarrow Z$ the natural projections. For a reproducing $(-*)$-kernel $K: Z \times Z \rightarrow \operatorname{Hom}\left(p_{2}^{*} \Pi, p_{1}^{*} \Pi\right)$ let $\mathcal{H}^{K}$ be the corresponding reproducing kernel Hilbert space, and define

$$
\widehat{K}: D \rightarrow \mathcal{H}^{K}, \quad \widehat{K}(\xi)=K_{\xi}=K(\cdot, \Pi(\xi)) \xi: Z \rightarrow D,
$$

and

$$
\zeta_{K}: Z \rightarrow \operatorname{Gr}\left(\mathcal{H}^{K}\right), \quad \zeta_{K}(s):=\overline{\widehat{K}\left(D_{s}\right)}
$$

Assume that we have an involutive morphism $\Omega=(\gamma, \omega)$ on $\Pi_{\mathcal{H}}$ such that the involutive diffeomorphism $\mathcal{S} \mapsto \mathcal{S}^{-*}:=\omega(\mathcal{S})$ of $\operatorname{Gr}\left(\mathcal{H}^{K}\right)$ satisfies

$$
(\forall s \in Z) \quad \zeta_{K}\left(s^{-*}\right)=\zeta_{K}(s)^{-*} .
$$

Then there exists a vector bundle morphism $\Delta_{K}=\left(\delta_{K}, \zeta_{K}\right)$ from $\Pi$ into the like-Hermitian vector bundle $\Pi_{\mathcal{H}^{K}, \Omega}$ such that $K$ is equal to the pull-back of the reproducing $(-*)$-kernel $Q_{\mathcal{H}^{K}, \Omega}$ by $\Delta_{K}$, that is, $K=\left(\Delta_{K}\right)^{*} Q_{\mathcal{H}^{K}, \Omega}$.

Proof. Denote

$$
L_{K}:=\left\{(s, x) \in Z \times \mathcal{H}^{K} \mid x \in \zeta_{K}(s)\right\} \subseteq Z \times \mathcal{H}^{K},
$$

and define

$$
\Lambda_{K}: L_{K} \rightarrow Z, \quad(s, x) \mapsto s .
$$

It follows by (5.1) that $\Lambda_{K}: L_{K} \rightarrow Z$ is a like-Hermitian vector bundle, which is just the pull-back of the tautological bundle $\Pi_{\mathcal{H}^{K}, \Omega}: \mathcal{T}\left(\mathcal{H}^{K}\right) \rightarrow \operatorname{Gr}\left(\mathcal{H}^{K}\right)$ by the mapping $\zeta_{K}: Z \rightarrow \operatorname{Gr}\left(\mathcal{H}^{K}\right)$.

On the other hand, define

$$
\check{K}: D \rightarrow L_{K}, \quad \check{K}(\xi)=(\Pi(\xi), \widehat{K}(\xi))=\left(\Pi(\xi), K_{\xi}\right)
$$

and

$$
\psi_{K}: L_{K} \rightarrow \mathcal{T}\left(\mathcal{H}^{K}\right), \quad \psi_{K}(s, x)=\left(\zeta_{K}(s), x\right)
$$


Then we get the commutative diagram

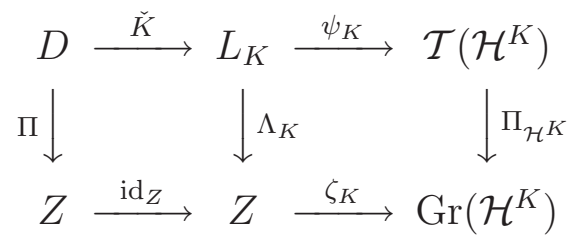

where the vertical arrows are like-Hermitian vector bundles, while the pairs of horizontal arrows $\left(\check{K}, \mathrm{id}_{Z}\right)$ and $\left(\psi_{K}, \zeta_{K}\right)$ are homomorphisms between these bundles.

We now define $\delta_{K}=\psi_{K} \circ \check{K}$ and check that the vector bundle homomorphism $\Delta_{K}:=\left(\delta_{K}, \zeta_{K}\right)$ has the wished-for property $K=\left(\Delta_{K}\right)^{*} Q_{\mathcal{H}^{K}, \Omega}$. In fact, let $t, s \in Z$ and $\eta \in D_{t}, \xi \in D_{s}$ arbitrary. Then

$$
\begin{aligned}
& \left(\left(\left(\Delta_{K}\right)^{*} Q_{\mathcal{H}^{K}, \Omega}\left(s^{-*}, t\right) \eta \mid \xi\right)_{s^{-*}, s}\right. \\
& \left.=\left(\left(\left.\delta_{K}\right|_{D_{s}}\right)^{-*} \circ Q_{\mathcal{H}^{K}, \Omega}\left(\zeta_{K}\left(s^{-*}\right), \zeta_{K}(t)\right) \circ\left(\left.\delta_{K}\right|_{D_{t}}\right)\right) \eta \mid \xi\right)_{s^{-*}, s} \\
& =\left(Q_{\mathcal{H}^{K}, \Omega}\left(\zeta_{K}\left(s^{-*}\right), \zeta_{K}(t)\right) \delta_{K} \eta \mid \delta_{K} \xi\right)_{\zeta_{K}(s)^{-*}, \zeta_{K}(s)} \\
& =\left(Q_{\mathcal{H}^{K}, \Omega}\left(\zeta_{K}\left(s^{-*}\right), \zeta_{K}(t)\right) K_{\eta} \mid K_{\xi}\right)_{\zeta_{K}(s)^{-*}, \zeta_{K}(s)} \\
& =\left(\left(p_{\zeta_{K}\left(s^{-*}\right)} \circ \gamma_{\zeta_{K}(t)}\left(K_{\eta}\right) \mid \gamma_{\zeta_{K}(s)}\left(K_{\xi}\right)\right)_{\mathcal{H}^{K}}\right. \\
& =\left(\gamma_{\zeta_{K}(t)}\left(K_{\eta}\right) \mid \gamma_{\zeta_{K}(s)}\left(K_{\xi}\right)\right)_{\mathcal{H}^{K}} \\
& =\left(K_{\eta} \mid K_{\xi}\right)_{\mathcal{H}^{K}}=\left(K\left(s^{-*}, t\right) \eta \mid \xi\right)_{s^{-*}, s}
\end{aligned}
$$

so that indeed $K=\left(\Delta_{K}\right)^{*} Q_{\mathcal{H}^{K}, \Omega}$.

The construction of the mapping $\zeta_{K}: Z \rightarrow \operatorname{Gr}\left(\mathcal{H}^{K}\right)$ in Theorem 5.1 is inspired by the mapping $\mathcal{Z}$ defined in formula (16) in [22], which in turn extends the corresponding maps for complex line bundles given in [25]. In the latter reference it is shown that categories formed by objects like $\zeta_{K}$ (or $\mathcal{Z}$ ) are equivalent to categories of vector bundles with distinguished kernels. Next, and under natural conditions, we extend that result by showing that the pull-back operation giving the kernel $K$ in Theorem 5.1 also enables us to recover the whole vector bundle $\Pi$.

Corollary 5.2 Under the conditions of Theorem 5.1, if, in addition, we assume that for every $s \in Z$

$$
K(s, s): D_{s} \rightarrow D_{s} \text { is invertible, }
$$

then the vector bundle morphism $\left(\check{K}, \mathrm{id}_{Z}\right)$ is an (algebraic) isomorphism between the bundles $\Pi: D \rightarrow Z$ and $\Lambda_{K}: L_{K} \rightarrow Z$, so $\Pi: D \rightarrow Z$ is isomorphic to the pull-back bundle $\zeta_{K}^{*} \mathcal{T}\left(\mathcal{H}^{K}\right) \rightarrow Z$ defined by the mapping $\zeta_{K}$. 
Proof. In view of Theorem 5.1 it suffices to show that the map $\check{K}$ has an inverse. If $(s, x) \in L_{K}$ then $x \in \zeta_{K}(s)=\widehat{K}\left(D_{s}\right)$ with $\widehat{K}$ injective, so there exists a unique $\xi \in D_{s}$ with $x=\widehat{K}(\xi)$. Then we obtain the inverse of $\check{K}$ by defining $\check{K}^{-1}: L_{K} \rightarrow D$ by $\check{K}^{-1}(s, x):=\left(\left.\widehat{K}\right|_{D_{s}}\right)^{-1}(x)$ for all $s \in Z$ and $x \in \zeta_{K}(s)$.

Now, of course, there is the question of the continuity and/or smoothness properties of the pull-back morphism $\Delta_{K}$ in the preceding theorem. Since $\Delta_{K}=\left(\delta_{K}, \zeta_{K}\right)$ with $\delta_{K}(\xi)=\left(\zeta_{K}(\Pi(\xi)), \widehat{K}(\xi)\right)$ for all $\xi \in D$, the morphism $\Delta_{K}$ is continuous if and only if $\widehat{K}$ and $\zeta_{K}$ are continuous mappings. In order to prove the continuity of $\widehat{K}$ it seems natural to assume the continuity of $K$.

For a trivialization $\Psi_{U}$ of $\Pi$ over the open set $U \subseteq Z$, let us denote $\Psi_{U}^{-1}(\xi)=\left(\Pi(\xi), \varphi_{U}(\xi)\right)$, so that $\varphi_{U}: D_{\Pi(\xi)} \rightarrow \mathcal{E}$ is a linear topological isomorphism, for all $\xi \in \Pi^{-1}(U)$. We shall sometimes identify $\xi$ with $\left(\Pi(\xi), \varphi_{U}(\xi)\right)$ putting $\xi \equiv\left(\Pi(\xi), \varphi_{U}(\xi)\right)$. Let $\Pi_{p_{2}^{*} p_{1}^{*}}$ denote the vector bundle $\operatorname{Hom}\left(p_{2}^{*} \Pi, p_{1}^{*} \Pi\right) \rightarrow Z \times Z$. If $U, V$ are open sets in $Z$ of corresponding trivializations $\Psi_{U}, \Psi_{V}$ one has

$$
\Pi_{p_{2}^{*} p_{1}^{*}}^{-1}(U \times V)=\bigcup_{(s, t) \in U \times V} \mathcal{B}\left(D_{t}, D_{s}\right)
$$

with associated isomorphisms

$$
\Psi_{U \times V}^{-1}: T \mapsto\left(s, t, T_{\mathcal{E}}\right), \Pi_{p_{2}^{*} p_{1}^{*}}^{-1}(U \times V) \rightarrow U \times V \times \mathcal{B}(\mathcal{E}, \mathcal{E})
$$

given by

$$
T_{\mathcal{E}}(x)=\varphi_{U}\left(T\left(\Psi_{V}(t, x)\right)\right), \quad(x \in \mathcal{E}),
$$

for $T \in \mathcal{B}\left(D_{t}, D_{s}\right)$, and

$$
\Psi_{U \times V}:\left(s, t, T_{\mathcal{E}}\right) \mapsto T_{s t}(\eta), U \times V \times \mathcal{B}(\mathcal{E}, \mathcal{E}) \rightarrow \Pi_{p_{2}^{*} p_{1}^{*}}^{-1}(U \times V)
$$

such that

$$
T_{s t}(\eta)=\Psi_{U}\left(s, T_{\mathcal{E}}\left(\varphi_{U}(\eta)\right)\right), \quad\left(\eta \in D_{t}\right) .
$$

The basic neighborhoods of any $T: D_{t} \rightarrow D_{s}$ of $\operatorname{Hom}\left(p_{2}^{*} \Pi, p_{1}^{*} \Pi\right)$ have the form $\Psi_{U \times V}\left(U_{0} \times V_{0} \times B_{\rho}\left(T_{\mathcal{E}}\right)\right)$ where $U, V$ are trivialization open subsets of $Z, U_{0} \subseteq U$ and $V_{0} \subseteq V$ run over the family of basic neighborhoods of $t, s$, respectively, and $B_{\rho}:=\left\{L \in \mathcal{B}(\mathcal{E}, \mathcal{E}) \mid\|L\|_{\mathcal{E} \rightarrow \mathcal{E}}<\rho\right\}$ with $B_{\rho}\left(T_{\mathcal{E}}\right)=T_{\mathcal{E}}+B_{\rho}$, for $\rho>0$.

If $K$ is a reproducing (-*)- kernel and $U, V$ are as above, we put

$$
K_{\mathcal{E}}^{U, V}(s, t):=\left.\Psi_{U \times V}^{-1} \circ K\right|_{U \times V}(s, t), \quad(s, t) \in U \times V .
$$

We shall identify $K_{\mathcal{E}}^{U, V}(s, t)$ with $K(s, t)_{\mathcal{E}}$ for all $(s, t) \in U \times V$. Clearly, $K$ is continuous if and only if $K_{\mathcal{E}}$ is. 
Lemma 5.3 If the reproducing kernel $K$ is continuous then the mapping

$$
\widehat{K}: D \rightarrow \mathcal{H}^{K}, \quad \widehat{K}(\xi)=K_{\xi}=K(\cdot, \Pi(\xi)) \xi: Z \rightarrow D,
$$

is continuous.

Proof. First, take $s_{0} \in Z$ and a trivialization isomorphism $\Psi_{U}$ with $s_{0} \in U$. For any $t \in U$, define the continuous sesquilinear form $(\cdot \mid \cdot)_{U, t}$ by

$$
(x \mid y)_{U, t}=\left(\Psi_{U}(t, x) \mid \Psi_{U^{-*}}\left(t^{-*}, y\right)\right)_{t, t^{-*}}, \quad \text { for } x, y \in \mathcal{E} .
$$

For $x, y \in \mathcal{E}$ and $t$ near $s_{0}$ there is a constant $C_{U}(x, y)>0$ such that $\left|(x \mid y)_{U, t}\right| \leq C_{U}(x, y)$, by property (c) in Definition 1.1. Then by using the Banach-Steinhaus theorem we get a constant $C_{U}>0$ such that

$$
\left|(x \mid y)_{U, t}\right| \leq C_{U}\|x\|\|y\|, \quad \text { for } x, y \in \mathcal{E}, t \in U^{\prime},
$$

for a suitable neighborhood $U^{\prime} \subseteq U$ of $s_{0}$.

Now, given $\rho>0$, since $K$ is continuous there exists an open set $U_{0} \subseteq U^{\prime}$ with $s_{0} \in U_{0}$, such that

$$
K\left(U_{0}^{-*}, U_{0}\right) \subset \Psi_{U^{-*} \times U}\left(U^{-*} \times U \times \Omega_{\rho}\left(K_{\mathcal{E}}^{U^{-*}, U}\left(s_{0}^{-*}, s_{0}\right)\right)\right) .
$$

Put $K_{\mathcal{E}}=K_{\mathcal{E}}^{U^{-*}, U}$. Then $\left\|K_{\mathcal{E}}\left(s^{-*}, t\right)-K_{\mathcal{E}}\left(s_{0}^{-*}, s_{0}\right)\right\| \leq \rho$ for $s, t \in U_{0}$, by the above inclusion. Finally, let $\xi_{0}=\Psi_{U}\left(s_{0}, x_{0}\right) \in D$, so $s_{0}=\Pi\left(\xi_{0}\right)$. Put $M=C_{U}\left(\left\|K_{\mathcal{E}}\left(s_{0}^{-*}, s_{0}\right)\right\|+\left\|x_{0}\right\|+2\right)\left(2\left\|x_{0}\right\|+1\right)$. If $\eta=(t, x) \in \Pi^{-1}\left(U_{0}\right)$ such that $x \in B_{\rho}\left(x_{0}\right):=\left\{y:\left\|y-x_{0}\right\| \leq \rho\right\}$ with $0<\rho<1$, then

$$
\begin{aligned}
\| K_{\eta} & -K_{\xi_{0}} \|^{2} \\
= & \left(K_{\eta} \mid K_{\eta}\right)-\left(K_{\xi_{0}} \mid K_{\eta}\right)-\left(K_{\eta} \mid K_{\xi_{0}}\right)+\left(K_{\xi_{0}} \mid K_{\xi_{0}}\right) \\
= & \left(K\left(t^{-*}, t\right) \eta-K\left(t^{-*}, s_{0}\right) \xi_{0} \mid \eta\right)_{t^{-*}, t} \\
& +\left(K\left(s_{0}^{-*}, s_{0}\right) \xi_{0}-K\left(s_{0}^{-*}, t\right) \eta \mid \xi_{0}\right)_{s_{0}^{-*}, s_{0}} \\
= & \left(K_{\mathcal{E}}\left(t^{-*}, t\right) x-K_{\mathcal{E}}\left(t^{-*}, s_{0}\right) x_{0} \mid x\right)_{U^{-*}, t^{-*}} \\
& +\left(K_{\mathcal{E}}\left(s_{0}^{-*}, s_{0}\right) x_{0}-K_{\mathcal{E}}\left(s_{0}^{-*}, t\right) x \mid x_{0}\right)_{U^{-*}, s_{0}^{-*}} \\
\leq & C_{U}\left(\left\|K_{\mathcal{E}}\left(t^{-*}, t\right)-K_{\mathcal{E}}\left(t^{-*}, s_{0}\right)\right\|\|x\|+\left\|K_{\mathcal{E}}\left(t^{-*}, s_{0}\right)\right\|\left\|x-x_{0}\right\|\right)\|x\| \\
& +C_{U}\left(\left\|K_{\mathcal{E}}\left(s_{0}^{-*}, s_{0}\right)\right\|\left\|x-x_{0}\right\|+\left\|K_{\mathcal{E}}\left(s_{0}^{-*}, s_{0}\right)-K_{\mathcal{E}}\left(s_{0}^{-*}, t\right)\right\|\|x\|\right)\left\|x_{0}\right\| \\
\leq & C_{U}\left(\rho\left(\rho+\left\|x_{0}\right\|\right)+\left(\rho+\left\|K_{\mathcal{E}}\left(s_{0}^{-*}, s_{0}\right)\right\|\right) \rho\right)\left(\rho+\left\|x_{0}\right\|\right) \\
& +C_{U}\left(\left\|K_{\mathcal{E}}\left(s_{0}^{-*}, s_{0}\right)\right\| \rho+\rho\left(\rho+\left\|x_{0}\right\|\right)\right)\left\|x_{0}\right\| \\
\leq & M \rho .
\end{aligned}
$$

Since this inequality holds for every $\eta$ in the neighborhood $\Psi_{U}\left(U_{0} \times B_{\rho}\left(x_{0}\right)\right)$ we have proved that $\widehat{K}$ is continuous. 
Remark 5.4 The argument used in Lemma 5.3 gives us in fact that the mapping

$$
(\xi, \eta) \mapsto K(\Pi(\xi), \Pi(\eta)) \eta, D \times D \rightarrow D
$$

is continuous provided that $K$ is continuous.

In the statement of Theorem 5.5 we are going to use the following terminology: Two linear subspaces $\mathcal{V}_{1}$ and $\mathcal{V}_{2}$ of some Hilbert space $\mathcal{H}$ are said to be similar to each other in $\mathcal{H}$ if there exists an invertible bounded linear operator $T: \mathcal{H} \rightarrow \mathcal{H}$ such that $T\left(\mathcal{V}_{1}\right)=\mathcal{V}_{2}$. For instance, it is easy to see that if $\mathcal{V}_{1}$ is finite-dimensional, then it is similar to $\mathcal{V}_{2}$ if and only if $\mathcal{V}_{2}$ is also finite-dimensional and $\operatorname{dim} \mathcal{V}_{1}=\operatorname{dim} \mathcal{V}_{2}$. Note however that if both $\mathcal{V}_{1}$ and $\mathcal{V}_{2}$ are closed infinite-dimensional subspaces of some separable Hilbert space $\mathcal{H}$ and $\operatorname{dim} \mathcal{V}_{1}^{\perp}<\infty=\operatorname{dim} \mathcal{V}_{2}^{\perp}$, then $\mathcal{V}_{1}$ and $\mathcal{V}_{2}$ are not similar to each other although $\operatorname{dim} \mathcal{V}_{1}=\operatorname{dim} \mathcal{V}_{2}(=\infty)$. In the theorem we are dealing with the mapping $\zeta$, which is set-valued.

For a topological space $\mathcal{T}$, we say that a mapping $F: \mathcal{T} \rightarrow \operatorname{Gr}(\mathcal{H})$ is similar-valued if $F(\tau)$ is similar to $F(\sigma)$ for every $\tau, \sigma \in \mathcal{T}$. Then $F$ will be called locally similar-valued on $\mathcal{T}$ if for any point $\tau$ in $\mathcal{T}$ there is a neighborhood $V$ of $\tau$ such that $\left.F\right|_{V}$ is similar-valued on $V$.

Since every orbit in $\operatorname{Gr}(\mathcal{H})$ is an open set (see for example Corollary 8.4(iv) in [28]), it is clear that a function $F$ as above is locally similar whenever it is continuous. Conversely, we are going to see that the continuity of the $\operatorname{Gr}\left(\mathcal{H}^{K}\right)$-valued mapping $\zeta_{K}$ is equivalent to its local similarity, under the assumption that $\widehat{K}$ is open on fibers.

Theorem 5.5 In the above setting, suppose that:

1. The kernel $K$ is continuous,

2. the bounded linear operator $\left.\widehat{K}\right|_{D_{s}}: D_{s} \rightarrow \mathcal{H}^{K}$ is injective with closed range for all $s \in Z$.

Then $\zeta_{K}: Z \rightarrow \operatorname{Gr}\left(\mathcal{H}^{K}\right)$ is continuous if and only if it is locally similar.

Proof. As noticed before, the continuity of $\zeta_{K}$ implies automatically that $\zeta_{K}$ is locally similar $\operatorname{Gr}(\mathcal{H})$-valued. Conversely, assume that $\zeta_{K}$ is locally similar. Let $s_{0} \in Z$ arbitrary and pick any open neighborhood $V \subseteq Z$ of $s_{0}$ such that there exists a trivialization $\Psi_{0}: V \times \mathcal{E} \rightarrow \Pi^{-1}(V)$ of the bundle $\Pi$ over $V$. Also denote $\mathcal{H}_{0}=\zeta_{K}\left(s_{0}\right) \subseteq \mathcal{H}^{K}$ and define the mapping

$$
\widetilde{K}_{V}: V \rightarrow \mathcal{B}\left(\mathcal{E}, \mathcal{H}^{K}\right), \quad s \mapsto \widehat{K}\left(\Psi_{0}(s, \cdot)\right) .
$$


Using an argument as that one of the proof of Lemma 5.3 it can be seen that the mapping $\widetilde{K}_{V}$ is continuous with respect to the norm operator topology: For $s, t \in V, x \in \mathcal{E}$ and $\xi \equiv(s, x), \eta \equiv(t, x)$,

$$
\begin{aligned}
\| \widetilde{K}_{V}(t) & x-\widetilde{K}_{V}(s) x \|^{2} \\
= & \left(K_{\mathcal{E}}\left(t^{-*}, t\right) x-K_{\mathcal{E}}\left(t^{-*}, s\right) x \mid x\right)_{V^{-*}, t^{-*}} \\
& +\left(K_{\mathcal{E}}\left(s^{-*}, s\right) x-K_{\mathcal{E}}\left(s^{-*}, t\right) x \mid x\right)_{V^{-*}, s^{-*}} \\
\leq & C_{V}\left(\left\|K_{\mathcal{E}}\left(t^{-*}, t\right)-K_{\mathcal{E}}\left(t^{-*}, s_{0}\right)\right\|+\left\|K_{\mathcal{E}}\left(s_{0}^{-*}, s_{0}\right)-K_{\mathcal{E}}\left(s_{0}^{-*}, t\right)\right\|\right)\|x\|^{2}
\end{aligned}
$$

from which $\lim _{t \rightarrow s}\left\|\widetilde{K}_{V}(t)-\widetilde{K}_{V}(s)\right\|_{\mathcal{E} \rightarrow \mathcal{H}^{K}}=0$.

Now, note that the mapping $\left.\widehat{K}\right|_{D_{s}}$ being injective with closed range means that for every $s \in Z$ there is a constant $C(s)>0$ such that for all $\xi \in D_{s}$ we have $\|\widehat{K}(\xi)\|^{2} \geq C(s)\|\xi\|_{D_{s}}^{2}$. This implies that $\widehat{K}\left(D_{s}\right)$ is a closed linear subspace of $\mathcal{H}^{K}$, so that $\zeta_{K}(s)=\widehat{K}\left(D_{s}\right)$, and moreover, for every $s \in V$ the operator $\widetilde{K}_{V}(s): \mathcal{E} \rightarrow \mathcal{H}^{K}$ is injective and its range is the closed subspace $\zeta_{K}(s) \subseteq \mathcal{H}^{K}$. For the sake of simplicity let us denote by $\widetilde{K}_{V}\left(s_{0}\right)^{-1}: \mathcal{H}_{0} \rightarrow \mathcal{E}$ the inverse of the bijective linear operator $x \mapsto \widetilde{K}_{V}\left(s_{0}\right) x, \mathcal{E} \rightarrow \mathcal{H}_{0}$.

Since $\mathcal{H}_{0}$ is a closed subspace of $\mathcal{H}^{K}$, it follows by the open mapping theorem that $\widetilde{K}_{V}\left(s_{0}\right)^{-1}$ is continuous, hence we can define

$$
\widehat{K}_{0}: V \rightarrow \mathcal{B}\left(\mathcal{H}_{0}, \mathcal{H}^{K}\right), \quad s \mapsto \widetilde{K}_{V}(s) \circ \widetilde{K}_{V}\left(s_{0}\right)^{-1} .
$$

This mapping is continuous with respect to the norm operator topology since so is $\widetilde{K}_{V}$. In addition, for every $s \in V$ we have $\operatorname{Ran}\left(\widetilde{K}_{V}(s)\right)=\zeta_{K}(s)$, hence the hypothesis implies that the ranges of the values of $\widehat{K}_{0}$ are similar to each other in $\mathcal{H}^{K}$. Since the mapping $T \mapsto \operatorname{Ran} T$ is continuous on the latter subset of $\mathcal{B}\left(\mathcal{H}_{0}, \mathcal{H}^{K}\right)$ by Proposition 3.6 in [9] (see also Example 6.1 in [10] and the principal bundle (4.5) in [11]), it then follows that the mapping $\zeta_{K}$ is continuous on $V$. Since $V$ is a neighborhood of the arbitrary point $s_{0} \in Z$, this completes the proof.

Remark 5.6 As regards the continuity of the mapping $T \mapsto \operatorname{Ran} T$ on certain sets of operators (see the final argument in the above proof), a related result belonging to this circle of ideas is provided by Proposition 1.7 in [20].

Remark 5.7 According to condition (2) assumed in Theorem 5.5, the operator $\left.K\right|_{D_{s}}: D_{s} \rightarrow \mathcal{H}^{K}$, being injective and with closed range, provides a topological isomorphism of $D_{s}$ onto a closed subspace of $\mathcal{H}^{K}$. Thus the topology of the fiber $D_{s}$ can be defined by a suitable scalar product which turns this fiber into a complex Hilbert space. In particular, this remark shows 
that the existence of a reproducing (-*)-kernel $K: Z \times Z \rightarrow \operatorname{Hom}\left(p_{2}^{*} \Pi, p_{1}^{*} \Pi\right)$ satisfying condition (2) at some point in the base of a like-Hermitian vector bundle $\Pi: D \rightarrow Z$ imposes rather strong conditions on this bundle — at least in the sense that the fiber of the bundle has to allow for a scalar product which defines its topology. (That is, the fiber has to be a Hilbertable vector space, in the terminology of Chapter VII in [21].) Thus condition (2) in Theorem 5.5 could appear as being rather restrictive. However, the next theorem shows that the invertibility property (5.2) of the kernel $K$ assumed in Corollary 5.2 is sufficient to imply that condition.

Theorem 5.8 Let $K: Z \times Z \rightarrow \operatorname{Hom}\left(p_{2}^{*} \Pi, p_{1}^{*} \Pi\right)$ be a reproducing (-*)kernel on a like-Hermitian vector bundle $\Pi: D \rightarrow Z$ as in Theorem 5.1, with the corresponding bundle morphism $\Delta_{K}$. Assume also that:

1. $K$ is continuous.

2. For every $s \in Z$,

$$
K(s, s): D_{s} \rightarrow D_{s} \text { is invertible. }
$$

3. The mapping $\zeta_{K}$ is locally similar in $\operatorname{Gr}\left(\mathcal{H}^{K}\right)$.

Then the morphism $\Delta_{K}$ is continuous.

Proof. Recall that $\Delta_{K}=\left(\delta_{K}, \zeta_{K}\right)$ and $\delta_{K}=\left(\zeta_{K} \circ \Pi, \widehat{K}\right)$. By Lemma 5.3, the continuity of $\widehat{K}$ follows by the fact that $K$ is assumed to be continuous. Then it is clear that the only thing we need to show is that point (2) of the statement implies point (2) of Theorem 5.5. To this end let $s \in Z$. Now observe that since the pair $(\cdot \mid \cdot)_{s^{-*}, s}$ is a strong duality pairing (see Remark 1.2) there exists a bounded linear operator $\theta_{s^{-*}}: \mathcal{H}^{K} \rightarrow D_{s}$ satisfying

$$
(\widehat{K}(\eta) \mid h)_{\mathcal{H}^{K}}=\left(\eta \mid \theta_{s^{-*}} h\right)_{s^{-*}, s} \quad \forall \eta \in D_{s}, \forall h \in \mathcal{H}^{K},
$$

see Lemma 3.5 in [3]. In fact, it turns out that the operator $\theta_{s^{-*}}$ restricted to $\mathcal{H}_{0}^{K}$ coincides with the evaluation mapping at $s$, see the proof of Proposition 3.7 in [3]. Thus under the assumption (5.4) we get, for every $\xi \in D_{s}$,

$$
\begin{aligned}
\|\xi\|^{2} & \leq\left\|K(s, s)^{-1}\right\|^{2}\|K(s, s) \xi\|_{D_{s}}^{2}=\left\|K(s, s)^{-1}\right\|^{2}\left\|\theta_{s^{-*}}\left(K_{\xi}\right)\right\|_{D_{s}}^{2} \\
& \leq C(s)\|\widehat{K}(\xi)\|_{\mathcal{H}^{K}}^{2} .
\end{aligned}
$$

This implies that $\left.\widehat{K}\right|_{D_{s}}: D_{s} \rightarrow \mathcal{H}^{K}$ is injective with closed range for all $s \in Z$, and the theorem follows. 
Remark 5.9 Assumptions (1) and (2) in the preceding theorem are automatically satisfied in many important situations: for instance, when $\Pi$ is a line bundle and $K(s, s) \neq 0$, or when $K=K^{\mathcal{R}}$ is the canonical kernel associated with a bundle in the category $\mathbf{R e p G L H}$, and with a transfer mapping $\mathcal{R}$. In fact, in this last case, if $s \in Z, \xi \in D_{s}$ and $\eta \in D_{s^{-*}}$ then for all $s \in Z$ we have $K(s, s)=$ id since

$$
\begin{aligned}
(K(s, s) \xi \mid \eta)_{s, s^{-*}} & =\left(\left(\mathcal{R}_{s^{-*}}\right)^{-*}\left(\mathcal{R}_{s} \xi\right) \mid \eta\right)_{s, s^{-*}}=\left(\mathcal{R}_{s} \xi \mid \mathcal{R}_{s^{-*}} \eta\right)_{\mathcal{H}} \\
& =(\xi \mid \eta)_{s, s^{-*}}
\end{aligned}
$$

In these cases the typical fiber of the corresponding bundle is Hilbertable, see Remark 5.7.

Remark 5.10 The proof of Corollary 5.2 only requires the injectivity of $\widehat{K}$ on $D_{s}, s \in Z$. So the corollary remains true if one replaces (5.2) with the weaker condition (2) of Theorem 5.5.

Assumption (3) in Theorem 5.8 holds for objects $(\Pi, G)$ in the category GrLHer under a mild condition, as we are going to see: Let $(\Pi, G)$ be an object of the category GrLHer, with $\Pi: D \rightarrow Z$ under the action of $G$ through $\mu: G \times D \rightarrow D$ and $\nu: G \times Z \rightarrow Z$. Let $K$ be a (-*)-reproducing kernel on $(\Pi, G)$ in the sense of Definition 2.3.

Lemma 5.11 For $\zeta_{K}(s)=\overline{\widehat{K}\left(D_{s}\right)}, s \in Z$, we have

$$
u \cdot \zeta_{K}(s)=\zeta_{K}(\nu(u, s)) \quad \forall u \in G, \forall s \in Z .
$$

Proof. By definition $\Pi(\mu(u, \xi))=\nu(u, \Pi(\xi))$ for all $u \in G$ and $\xi \in D$. From this, it follows easily that $D_{\nu\left(u, s_{0}\right)}=\mu\left(u, D_{s}\right)$ for all $u \in G$ and $s \in Z$.

We now claim that the group $G$ is taken into $\mathcal{G}=\operatorname{GL}\left(\mathcal{H}^{K}\right)$ through the natural action (which we denote in this proof by ".") of $G$ on the section space $\Gamma(Z, D)$ of the bundle $\Pi$. So in particular $K_{\mu(u, \xi)}=u \cdot K_{\xi}$, for $u \in G$ and $\xi \in D_{s}$. To see this, notice that, given $u \in G$ and $s \in Z$, for any $t \in Z$ and $\eta \in D_{t}$,

$$
\begin{aligned}
\left(K_{\mu(u, \xi)} \mid K_{\eta}\right): & =\left(K\left(t^{-*}, \nu(u, s)\right) \mu(u, \xi) \mid \eta\right)_{t^{-*}, t} \\
& =\left(\mu\left(u, K\left(\nu\left(u^{-1}, t^{-*}\right), s\right) \xi\right) \mid \eta\right)_{t^{-*}, t} \\
& =\left(\mu\left(u, K_{\xi}\left(\nu\left(u^{-1}, t^{-*}\right)\right)\right) \mid \eta\right)_{t^{-*}, t} \\
& =\left(u \cdot K_{\xi} \mid K_{\eta}\right)
\end{aligned}
$$

by (2.3). By the density of the linear combinations of sections $K_{\eta}$ in $\mathcal{H}^{K}$ we obtain the wished-for relation $K_{\mu(u, \xi)}=u \cdot K_{\xi}$, for $u \in G$ and $\xi \in D_{s}$. 
Let $u \in G$ again. Since the action of $u$ on the space $\Gamma(Z, D)$ is continuous we have $u \cdot \widehat{\widehat{K}\left(D_{s}\right)} \subseteq \overline{u \cdot \widehat{K}\left(D_{s}\right)}$ and then

$$
u^{-1} \cdot \overline{u \cdot \widehat{K}\left(D_{s}\right)} \subseteq \overline{\left(u^{-1} u\right) \cdot \widehat{K}\left(D_{s}\right)}=\overline{\widehat{K}\left(D_{s}\right)} .
$$

Both inclusions give us $u \cdot \overline{\widehat{K}\left(D_{s}\right)}=\overline{u \cdot \widehat{K}\left(D_{s}\right)}$. In consequence,

$$
\zeta_{K}(\nu(u, s))=\overline{\widehat{K}\left(D_{\nu(u, s)}\right)}=\overline{\widehat{K}\left(\mu\left(u, D_{s}\right)\right)}=\overline{\widehat{u} \cdot K\left(D_{s}\right)}=u \cdot \zeta_{K}(s) .
$$

Corollary $\mathbf{5 . 1 2}$ Let $(\Pi, G)$ be an object in the category GrLHer, and let $K$ be a continuous reproducing kernel on $\Pi$ in this category. Suppose, in addition to the assumption of Theorem 5.1, that for every $s \in Z$ the isotropy group $G_{s}:=\{u \in G \mid \nu(u, s)=s\}$ is a Lie subgroup of $G$ and that the mapping $K(s, s)$ is invertible. Then the bundle morphism $\Delta_{K}$ is continuous.

Proof. In view of previous results, we only have to verify that the mapping $\zeta$ is locally similar. But this is a clear consequence of the above lemma, since the fact that $G_{s}$ is a Lie subgroup entails that $\{\nu(u, s) \mid u \in G\}$ is an open subset of $\mathrm{Z}$ for every $s \in Z$.

Remark 5.13 Let us discuss briefly the condition in Theorem 5.1 on the existence of a suitable involutive diffeomorphism of $\operatorname{Gr}\left(\mathcal{H}^{K}\right)$ onto itself in the case when the base $Z$ of the bundle $\Pi: D \rightarrow Z$ is connected.

Assuming that the mapping $\zeta_{K}: Z \rightarrow \operatorname{Gr}\left(\mathcal{H}^{K}\right)$ is continuous, the image of this map will be a connected subset of $\operatorname{Gr}\left(\mathcal{H}^{K}\right)$. In particular, $\zeta_{K}(Z)$ will be contained in a connected component of $\operatorname{Gr}\left(\mathcal{H}^{K}\right)$. It then follows by Remark 4.2(b) in [4] that there exists a closed linear subspace $\mathcal{S}_{0}$ of $\mathcal{H}$ such that $\zeta_{K}(Z) \subseteq \operatorname{Gr}_{\mathcal{S}_{0}}(\mathcal{H})$. So condition (5.1) of Theorem 5.1 actually requires a suitable involutive diffeomorphism only on the unitary orbit $\operatorname{Gr}_{\mathcal{S}_{0}}(\mathcal{H})$, not on the whole $\operatorname{Gr}\left(\mathcal{H}^{K}\right)$.

Thus the role of universal reproducing $(-*)$-kernel seems to be played by the reproducing $(-*)$-kernels induced by $Q_{\mathcal{H}}$ on the restrictions of the tautological vector bundle $\Theta_{\mathcal{H}}$ to the various unitary orbits $\operatorname{Gr}_{\mathcal{S}_{0}}(\mathcal{H})$ endowed with various $(-*)$-structures, for arbitrary Hilbert spaces $\mathcal{H}$.

Another situation when we are naturally led to deal with orbits $\operatorname{Gr}_{\mathcal{S}_{0}}(\mathcal{H})$ of Grassmannians is that one considered prior to Lemma 5.11, in the case when the action of the group $G$ on $Z$ is transitive. Under such a condition, we manage in the next result to remove the assumption about the existence of the involutive morphism $\Omega$ satisfying (5.1) (which is a highly non-canonical assumption). In order to do so, we must replace the tautological bundle $\Pi_{\mathcal{H}}$ by its complexification $\Pi_{\mathcal{H}}^{\mathrm{C}}$. 
Now let $(\Pi, G)$ be an object of the category GrLHer, as above, where $\Pi: D \rightarrow Z, \mu: G \times D \rightarrow D, \nu: G \times Z \rightarrow Z$, such that the action $\nu$ is transitive on $Z$. Assume that there exists $s_{0} \in Z$ with $s_{0}^{-*}=s_{0}$. Let $K$ be a reproducing $(-*)$-kernel on $\Pi$ satisfying the property $(2.3)$, and let $\mathcal{H}^{K}$ be its reproducing kernel Hilbert space. For $\widehat{K}$ as in Theorem 5.1, set $\mathcal{S}_{0}:=\overline{\widehat{K}\left(D_{s_{0}}\right)} \subseteq \mathcal{H}^{K}$. Recall that the vector bundle $\mathcal{G} \times{ }_{\mathcal{G}(p)} \mathcal{S}_{0} \rightarrow \mathcal{G} / \mathcal{G}(p)$ was denoted by $\Pi_{\mathcal{H}^{K}}^{\mathrm{C}}$. Here $p=p_{\mathcal{S}_{0}}$ and $\mathcal{G}=\mathrm{GL}\left(\mathcal{H}^{K}\right)$. Let in turn $Q_{\mathcal{H}^{K}}^{\mathrm{C}}$ denote the kernel associated with the bundle $\Pi_{\mathcal{H}^{K}}^{\mathrm{C}}$ and the projection $p$ as in the subsection 1.3.

Theorem 5.14 In the preceding setting, there exists a vector bundle morphism $\widetilde{\Delta}_{K}=\left(\tilde{\delta}_{K}, \tilde{\zeta}_{K}\right)$ from $\Pi$ into $\Pi_{\mathcal{H}^{K}}^{\mathbf{C}}$ such that $K$ is equal to the pull-back of the reproducing $(-*)$-kernel $Q_{\mathcal{H}^{K}}^{\mathrm{C}}$, that is,

$$
K=\left(\widetilde{\Delta}_{K}\right)^{*} Q_{\mathcal{H}^{K}}^{\mathbf{C}}
$$

In addition, if

1. the isotropy group at the point $s_{0}=s_{0}^{-*} \in Z$ is a complex Banach-Lie subgroup of $G$ and

2. the reproducing $(-*)$-kernel $K$ is holomorphic of the second kind, then the vector bundle morphism $\widetilde{\Delta}_{K}$ is holomorphic.

Proof. As it was seen in Lemma 5.11, $\zeta_{K}\left(\nu\left(u, s_{0}\right)\right)=u \cdot \zeta_{K}\left(s_{0}\right)$ for every $u \in G$. Let us proceed to constructing the morphism $\widetilde{\Delta}_{K}$. Define, for $s=\nu\left(u, s_{0}\right)$ in $Z$,

$$
\tilde{\zeta}_{K}(s):=\left(\zeta_{K}\left(\nu\left(u, s_{0}\right)\right), \zeta_{K}\left(\nu\left(u^{-*}, s_{0}\right)\right)\right)=\left(u \cdot \mathcal{S}_{0}, u^{-*} \cdot \mathcal{S}_{0}\right) \equiv u \mathcal{G}(p) .
$$

Then, on account of the definition of the involution $-*$ in $\mathcal{G}(p)$, we have

$$
\begin{aligned}
\tilde{\zeta}_{K}(s)^{-*} & =[u \cdot \mathcal{G}(p)]^{-*}:=u^{-*} \cdot \mathcal{G}(p)=\tilde{\zeta}_{K}\left(\nu\left(u^{-*}, s_{0}\right)\right)=\tilde{\zeta}_{K}\left(\nu\left(u, s_{0}\right)^{-*}\right) \\
& =\tilde{\zeta}_{K}\left(s^{-*}\right) .
\end{aligned}
$$

Take $\xi \in D$. Then $\xi \in D_{s}$ for some (unique) $s=\nu\left(u, s_{0}\right)$, where $u \in G$. Define $\tilde{\delta}_{K}(\xi)$ in $\mathcal{G} \times_{\mathcal{G}(p)} \mathcal{S}_{0}$ by

$$
\tilde{\delta}_{K}(\xi)=\left[\left(u, K_{\mu\left(u^{-1}, \xi\right)}\right)\right] .
$$

It is readily seen that $\tilde{\delta}_{K}$ is well defined and then that $\left(\tilde{\delta}_{K}, \tilde{\zeta}_{K}\right)$ is a morphism between the like-Hermitian vector bundles $\Pi$ and $\Pi_{\mathcal{H}_{K}}^{\mathbf{C}}$. Put for a while $\delta=\tilde{\delta}_{K}$ and $\zeta=\tilde{\zeta}_{K}$ for simplicity. 
For $s=\nu\left(u, s_{0}\right), t=\nu\left(v, s_{0}\right) \in Z$ and $\xi \in D_{s^{-*}}, \eta \in D_{t}$, we have

$$
\begin{aligned}
& \left(\left(\left(\delta_{s^{-*}}\right)^{-*} \circ Q_{\mathcal{H}^{K}}^{\mathbf{C}}(\zeta(s), \zeta(t)) \circ \delta_{t}\right) \eta \mid \xi\right)_{s, s^{-*}} \\
& =\left(\left(\delta_{s^{-*}}\right)^{-*}\left[\left(u, p\left(u^{-1} \cdot v \cdot K_{\mu\left(v^{-1}, \eta\right)}\right)\right)\right] \mid \xi\right)_{s, s^{-*}} \\
& =\left(\left[\left(u, p\left(u^{-1} \cdot v \cdot K_{\mu\left(v^{-1}, \eta\right)}\right)\right] \mid\left[u^{-*}, K_{\mu\left(u^{-*}, \xi\right)}\right)\right]\right)_{u \cdot \mathcal{G}(p), u^{-*} \cdot \mathcal{G}(p)} \\
& =\left(p_{\mathcal{S}_{0}}\left(u^{-1} \cdot v \cdot K_{\mu\left(v^{-1}, \eta\right)}\right) \mid K_{\mu\left(u^{-*}, \xi\right)}\right)_{\mathcal{H}^{K}}=(K(s, t) \eta \mid \xi)_{\mathcal{H}^{K}}
\end{aligned}
$$

This implies that $K=\left(\widetilde{\Delta}_{K}\right)^{*} Q_{\mathcal{H}^{K}}^{\mathbf{C}}$, that is, $K$ is the pull-back of $Q_{\mathcal{H}^{K}}^{\mathbf{C}}$ by the morphism $\widetilde{\Delta}_{K}$.

To prove the second part of the statement, recall that if the isotropy group at $s_{0} \in Z$ is a complex Banach-Lie subgroup of $G$, then the orbit mapping

$$
u \mapsto \nu\left(u, s_{0}\right), \quad G \rightarrow Z
$$

has holomorphic local cross-sections around every point $s \in Z$. Therefore, in the above construction of the vector bundle morphism $\widetilde{\Delta}_{K}=\left(\tilde{\delta}_{K}, \tilde{\zeta}_{K}\right)$ (see formulas (5.5) and (5.6)), the element $u \in G$ can be chosen to depend holomorphically on $s \in Z$ around an arbitrary point in $Z$. This remark implies at once that the mapping $\tilde{\zeta}_{K}$ is holomorphic and that in order to prove that the component $\tilde{\delta}_{K}$ is holomorphic as well, it will be enough to check that the mapping

$$
\widehat{K}: \xi \mapsto K_{\xi}, D \rightarrow \mathcal{H}^{K}
$$

is holomorphic. Since $K$ is continuous, by Lemma 5.3 we have that $\widehat{K}$ is continuous. Then, by Corollary A.III.3 in Appendix III of [23], it will be sufficient to show that for every $h \in \mathcal{H}_{0}^{K}$ the function $(\widehat{K}(\cdot) \mid h)_{\mathcal{H}^{K}}$ is holomorphic on $D$. Thus take $\xi, \eta \in D, s=\Pi(\xi), t=\Pi(\eta)$. Then, by $(2.2)$ and (2.1),

$$
\left(\widehat{K}(\xi) \mid K_{\eta}\right)_{\mathcal{H}^{K}}=\left(K\left(t^{-*}, s\right) \xi \mid \eta\right)_{t^{-*}, t} .
$$

Hence, by the hypothesis that the kernel $K$ is holomorphic of the second kind (see Definition 2.1), we obtain that $\widehat{K}: D \rightarrow \mathcal{H}^{K}$ is holomorphic, and this concludes the proof.

Remark 5.15 There is a close relationship between Theorem 3.8 and Theorem 5.14, and yet they are different from each other, for in Theorem 5.14 it is not assumed the existence of a transfer mapping (relating the bundle $\Pi: D \rightarrow Z$ with some Hilbert space $\mathcal{H}$ ). Instead, one needs to consider in Theorem 5.14 the Hilbert space of sections $\mathcal{H}^{K}$. Is there some transfer mapping, say $\mathcal{R}_{K}$, from $D$ into $\mathcal{H}^{K}$ ? A natural candidate seems to be the mapping $\mathcal{R}_{K}: \xi \mapsto\left[\left(u, K_{\mu\left(u^{-1}, \xi\right)}\right)\right] \mapsto u \cdot K_{\mu\left(u^{-1}, \xi\right)}$. Since for all $u \in G, \xi \in D$ we 
have $u \cdot K_{\mu\left(u^{-1}, \xi\right)}=K_{\xi}$ such a map is $\widehat{K}$. However $\widehat{K}$ need not be an isometry, or even injective, in general. (Recall that $(\widehat{K}(\xi) \mid \widehat{K}(\eta))_{\mathcal{H}^{K}}=(K(s, s) \xi \mid \eta)_{s, s^{-*}}$ for $s \in Z, \xi \in D_{s}, \eta \in D_{s^{-*}}$ )

\section{Applications of the pull-back operation on reproduc- ing $(-*)$-kernels}

The first application of the pull-back operation works out the precise relationship between reproducing $(-*)$-kernels on homogeneous vector bundles constructed as in the subsection 1.4. The resulting result, part (d), illustrates Proposition 3.6.

Proposition 6.1 Given two $C^{*}$-algebras $1 \in B \subseteq A$ with a conditional expectation $E: A \rightarrow B$, assume that we have a tracial state $\varphi: A \rightarrow \mathbb{C}$ (i.e., $\varphi\left(a_{1} a_{2}\right)=\varphi\left(a_{2} a_{1}\right)$ for every $a_{1} a_{2} \in A$ ) such that $\varphi \circ E=\varphi$. For $X \in\{A, B\}$ denote by $G_{X}$ the group of invertible elements of $X$, by $\mathcal{H}_{X}$ the Hilbert space obtained by the GNS construction out of the state $\left.\varphi\right|_{X}$ of $X$, and by $C_{X}: \mathcal{H}_{X} \rightarrow \mathcal{H}_{X}$ the antilinear isometric involutive operator defined by the involution of $X$. Also denote by $P: \mathcal{H}_{A} \rightarrow \mathcal{H}_{B}$ the orthogonal projection and by $\lambda, \rho, \pi: A \rightarrow \mathcal{B}\left(\mathcal{H}_{A}\right)$ the representations defined by

$\lambda(u)[f]=[u f], \quad \rho(u)[f]=\left[f u^{-1}\right], \quad$ and $\quad \pi(u)[f]=\lambda(u) \rho(u)[f]=\left[u f u^{-1}\right]$

whenever $u \in G_{B}$ and $f \in B$, where we denote by $f \mapsto[f]$ the natural map $B \rightarrow \mathcal{H}_{B}$.

Now for $\alpha \in\{\lambda, \rho, \pi\}$ denote by $K_{\alpha}$ the corresponding reproducing (-*)kernel on the homogeneous bundle $G_{A} \times{ }_{\alpha} \mathcal{H}_{B} \rightarrow G_{A} / G_{B}$. Then the following assertions hold:

(a) There exist well-defined diffeomorphisms

$$
\begin{gathered}
\delta_{1}: G_{A} \times{ }_{\lambda} \mathcal{H}_{B} \rightarrow G_{A} \times{ }_{\rho} \mathcal{H}_{B}, \quad \delta_{2}: G_{A} \times{ }_{\rho} \mathcal{H}_{B} \rightarrow G_{A} \times{ }_{\lambda} \mathcal{H}_{B} \\
\text { and } \delta_{3}: G_{A} \times{ }_{\pi} \mathcal{H}_{B} \rightarrow G_{A} \times{ }_{\pi} \mathcal{H}_{B},
\end{gathered}
$$

given, each of them, by the correspondence $[(u, f)] \mapsto\left[\left(u^{-*}, C_{B}(f)\right)\right]$.

(b) For $j=1,2,3$ denote by $\Theta_{j}$ the pair consisting of the mappings $\delta_{j}$ and $u G_{B} \mapsto u^{-*} G_{B}$. Then $\Theta_{j}$ is an adjointable antimorphism of likeHermitian bundles.

(c) We have $\Theta_{1}^{*} K_{\rho}=K_{\lambda}, \Theta_{2}^{*} K_{\lambda}=K_{\rho}$, and $\Theta_{3}^{*} K_{\pi}=K_{\pi}$.

(d) There exists an involutive antilinear isometry $\bar{\Theta}_{3}: \mathcal{H}_{\mathbb{C}}^{K_{\pi}} \rightarrow \mathcal{H}_{\mathbb{C}}^{K_{\pi}}$ such that $\bar{\Theta}_{3}\left(\left(K_{\pi}\right)_{\xi}\right)=\left(K_{\pi}\right)_{\tau(\xi)}$ for all $\xi \in D$. 
Proof. First of all, notice that $\varphi$ being tracial the representation $\rho$ is well defined, so is $\pi$. To prove assertion (a), note that for all $v \in G_{B}$ and $f \in B$ we have

$$
C_{B}\left(\lambda\left(v^{-1}\right)[f]\right)=\left[\left(v^{-1} f\right)^{*}\right]=\left[f^{*}\left(v^{*}\right)^{-1}\right]=\rho\left(\left(v^{-*}\right)^{-1}\right) C_{B}([f]) .
$$

Now, if $\left(u_{1}, f_{1}\right) \sim_{\lambda}\left(u_{2}, f_{2}\right)$ then there exists $v \in G_{B}$ such that $u_{2}=u_{1} v$ and $f_{2}=\lambda\left(v^{-1}\right) f_{1}$. Then the above calculation shows that $C_{B}\left(f_{2}\right)=$ $\rho\left(\left(v^{-*}\right)^{-1}\right) C_{B}\left(f_{1}\right)$, so that $\left(u_{1}^{-*}, C_{B}\left(f_{1}\right)\right) \sim_{\rho}\left(u_{2}^{-*}, C_{B}\left(f_{2}\right)\right)$. Thus the mapping $\delta_{1}: G_{A} \times{ }_{\lambda} \mathcal{H}_{B} \rightarrow G_{A} \times{ }_{\rho} \mathcal{H}_{B}$ is well defined.

Since the projection mappings $G_{A} \times \mathcal{H}_{B} \rightarrow G_{A} \times{ }_{\alpha} \mathcal{H}_{B}$ are submersions for $\alpha \in\{\lambda, \rho\}$ and the mapping $(u, f) \mapsto\left(u^{-*}, C_{B}(f)\right)$ is clearly a diffeomorphism of $G_{A} \times \mathcal{H}_{B}$ onto itself, it then follows that $\delta_{1}$ is smooth (see Corollary 8.4 in [28]). One can similarly check that $\delta_{2}: G_{A} \times{ }_{\rho} \mathcal{H}_{B} \rightarrow G_{A} \times_{\lambda} \mathcal{H}_{B}$ is smooth. In addition, it is easy to see that the mappings $\delta_{1}$ and $\delta_{2}$ are inverse to each other, hence they are diffeomorphisms.

The fact that $\delta_{3}$ is also a well-defined smooth mapping follows by a similar reasoning based on the calculation

$$
C_{B}\left(\pi\left(v^{-1}\right)[f]\right)=\left[\left(v^{-1} f v\right)^{*}\right]=\left[v^{*} f^{*} v^{-*}\right]=\pi\left(\left(v^{-*}\right)^{-1}\right) C_{B}([f])
$$

that holds whenever $v \in G_{B}$ and $f \in B$. Since $\delta_{3} \circ \delta_{3}=\operatorname{id}_{G_{A} \times_{\pi} \mathcal{H}_{B}}$, it then follows that $\delta_{3}$ is a diffeomorphism as well.

For assertion (b) we shall use Remark 1.6. It is clear that for $j=1,2,3$ the mapping $\delta_{j}$ is a fiberwise isomorphism of real Banach spaces, so that it will be enough to prove that for all $u \in G_{A}$ and $f \in \mathcal{H}_{B}$ we have

$$
\left([(u, f)] \mid\left[\left(u^{-*}, g\right)\right]\right)_{u G_{B}, u^{-*} G_{B}}=\overline{\left(\delta_{j}[(u, f)] \mid \delta_{j}\left[\left(u^{-*}, g\right)\right]\right)_{u^{-*} G_{B}, u^{-*} G_{B}}} .
$$

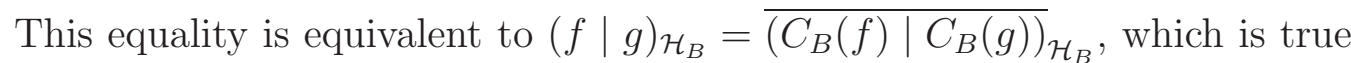
since $C_{B}: \mathcal{H}_{B} \rightarrow \mathcal{H}_{B}$ is an antilinear isometry.

Assertion (c) can be proved by means of (3.5). For instance, in order to check that $\Theta_{1}^{*} K_{\rho}=K_{\lambda}$, we need to prove that for all $u_{1}, u_{2} \in G_{A}$ and $f_{1}, f_{2} \in \mathcal{H}_{B}$ we have

$$
\begin{aligned}
& \left(K_{\lambda}\left(u_{1} G_{B}, u_{2} G_{B}\right)\left[\left(u_{2}, f_{2}\right)\right] \mid\left[\left(u_{1}^{-*}, f_{1}\right)\right]\right)_{u_{1} G_{B}, u_{1}^{-*} G_{B}}= \\
& =\overline{\left(K_{\rho}\left(u_{1}^{-*} G_{B}, u_{2}^{-*} G_{B}\right) \delta_{1}\left[\left(u_{2}, f_{2}\right)\right] \mid \delta_{1}\left[\left(u_{1}^{-*}, f_{1}\right)\right]\right)_{u_{1}^{-*} G_{B}, u_{1} G_{B}}} .
\end{aligned}
$$

In view of the way $\delta_{1}$ is defined, the right-hand side is equal to

$$
{\overline{\left(K_{\rho}\left(u_{1}^{-*} G_{B}, u_{2}^{-*} G_{B}\right)\left[\left(u_{2}^{-*}, C_{B}\left(f_{2}\right)\right)\right] \mid\left[\left(u_{1}, C_{B}\left(f_{1}\right)\right)\right]\right)}}_{u_{1}^{-*} G_{B}, u_{1} G_{B}} .
$$


Now, by taking into account the definitions of $K_{\lambda}$ and $K_{\rho}$ (see [3]), it follows that the wished-for equality is equivalent to

$$
\begin{aligned}
& \left(\left[\left(u_{1}, P\left(\lambda\left(u_{1}^{-1}\right) \lambda\left(u_{2}\right) f_{2}\right)\right)\right] \mid\left[\left(u_{1}^{-*}, f_{1}\right)\right]\right)_{u_{1} G_{B}, u_{1}^{-*} G_{B}}= \\
& \quad=\overline{\left(\left[\left(u_{1}^{-*}, P\left(\rho\left(\left(u_{1}^{-*}\right)^{-1}\right) \rho\left(u_{2}^{-*}\right) C_{B}\left(f_{2}\right)\right)\right)\right] \mid\left[\left(u_{1}, C_{B}\left(f_{1}\right)\right)\right]\right)_{u_{1}^{-*} G_{B}, u_{1} G_{B}}} .
\end{aligned}
$$

This is further equivalent to

$$
\left(P\left(\lambda\left(u_{1}^{-1} u_{2}\right) f_{2}\right) \mid f_{1}\right)_{\mathcal{H}_{B}}=\overline{\left(P\left(\rho\left(\left(\left(u_{1}^{-*}\right)^{-1} u_{2}^{-*}\right) C_{B}\left(f_{2}\right)\right) \mid C_{B}\left(f_{1}\right)\right)\right)_{\mathcal{H}_{B}}} .
$$

Now we have

$$
\begin{aligned}
\left(P \left(\rho\left(\left(\left(u_{1}^{-*}\right)^{-1} u_{2}^{-*}\right) C_{B}\left(f_{2}\right)\right) \mid\right.\right. & \left.C_{B}\left(f_{1}\right)\right)_{\mathcal{H}_{B}}= \\
= & \left(\rho\left(\left(\left(u_{1}^{-*}\right)^{-1} u_{2}^{-*}\right) C_{B}\left(f_{2}\right) \mid P\left(C_{B}\left(f_{1}\right)\right)\right)_{\mathcal{H}_{B}}\right. \\
& =\left(\rho\left(\left(\left(u_{1}^{-1} u_{2}\right)^{-*}\right) C_{B}\left(f_{2}\right) \mid C_{B}\left(f_{1}\right)\right)_{\mathcal{H}_{B}}\right. \\
& =\left(C_{B}\left(\lambda\left(\left(\left(u_{1}^{-1} u_{2}\right)^{-*}\right) f_{2}\right) \mid C_{B}\left(f_{1}\right)\right)_{\mathcal{H}_{B}}\right. \\
& =\left(f_{1} \mid \lambda\left(\left(\left(u_{1}^{-1} u_{2}\right)^{-*}\right) f_{2}\right) \mathcal{H}_{B}\right. \\
& =\left(f_{1} \mid P\left(\lambda\left(\left(\left(u_{1}^{-1} u_{2}\right)^{-*}\right) f_{2}\right)\right)_{\mathcal{H}_{B}}\right. \\
& =\overline{\left(P\left(\lambda\left(\left(\left(u_{1}^{-1} u_{2}\right)^{-*}\right) f_{2}\right) \mid f_{1}\right)\right.}
\end{aligned}
$$

where the third equality follows by (6.1). This shows that (6.2) holds and completes the proof of the fact that $\Theta_{1}^{*} K_{\rho}=K_{\lambda}$.

The equalities $\Theta_{2}^{*} K_{\lambda}=K_{\rho}$ and $\Theta_{3}^{*} K_{\pi}=K_{\pi}$ can be proved similarly.

To prove assertion (d), just note that it follows by (c) that (3.8) in Corollary 3.7 is satisfied.

As regards item (d) in the statement of Proposition 6.1, it is to be noticed that the diagram

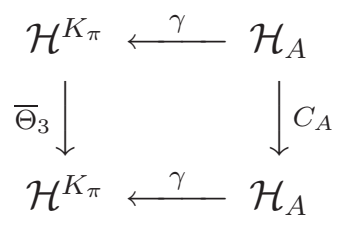

is commutative, where the mapping $\gamma$ is a suitable isometry (see [3] and the comments after Theorem 6.2).

The next application is a special case of Theorem 5.1 and yet it applies to all of the classical reproducing kernels (see for instance [15] and Example I.1.10 in [23] for many specific examples) when they are thought of as living on trivial line bundles. Moreover, note that the classical reproducing kernel spaces of complex functions on domains in $\mathbb{C}^{n}$ (Bergman spaces, Hardy spaces etc.) are separable Hilbert spaces hence are isomorphic 
to $\ell^{2}(\mathbb{N})$. Hence the reproducing kernels of all these classical function spaces can be obtained as pull-backs of a unique kernel, namely the universal reproducing kernel $Q_{\mathcal{H}}$ for $\mathcal{H}=\ell^{2}(\mathbb{N})$. See [24] and [25] for the implications of this fact in quantum mechanics.

Theorem 6.2 Let $\Pi: D \rightarrow Z$ be a Hermitian vector bundle and denote by $p_{1}, p_{2}: Z \times Z \rightarrow Z$ the natural projections. Let $K: Z \times Z \rightarrow \operatorname{Hom}\left(p_{2}^{*} \Pi, p_{1}^{*} \Pi\right)$ be a reproducing kernel. If $\mathcal{H}^{K}$ stands for the reproducing kernel Hilbert space associated with $K$, then there exists a vector bundle homomorphism $\Delta_{K}=\left(\delta_{K}, \zeta_{K}\right)$ from $\Pi$ into the tautological vector bundle $\Pi_{\mathcal{H}^{K}}$ such that $K$ is equal to the pull-back of $Q_{\mathcal{H}^{K}}$ by $\Delta_{K}$.

Proof. The hypothesis of Theorem 5.1 is clearly satisfied if we take $\Omega$ to be the identity morphism on the tautological vector bundle $\Pi_{\mathcal{H}}$.

Finding applications of Theorem 5.1 in other concrete cases involving general involutions $-*$, for which condition (5.1) is automatically fulfilled, does not seem simple. Instead, one has to use complexifications of bundles of the type $\mathcal{T}_{\mathcal{S}_{0}}(\mathcal{H}) \rightarrow \operatorname{Gr}_{\mathcal{S}_{0}}(\mathcal{H}), \mathcal{S}_{0} \in \operatorname{Gr}(\mathcal{H})$, as in Theorem 5.14. This theorem clearly applies to bundles $G_{A} \times_{G_{B}} \mathcal{H}_{B} \rightarrow G_{A} / G_{B}$ in the category HomogLHer and kernels $K^{\pi}$ accordingly, as in Remark 2.9. Let us now assume for simplicity, in that case, that $\mathcal{H}_{A}=\overline{\operatorname{span}} \pi_{A}\left(G_{A}\right) \mathcal{H}_{B}$. (This holds for instance when $A, B$ are $C^{*}$-algebras and $G_{A} \times_{G_{B}} \mathcal{H}_{B} \rightarrow G_{A} / G_{B}$ is an object in StLHer.) Then $\mathcal{H}_{A}$ and $\mathcal{H}^{K_{\pi}}$ are isomorphic Hilbert spaces via the unitary "realization" operator $\gamma: h \mapsto F_{h}, \mathcal{H}_{A} \rightarrow \mathcal{H}^{K_{\pi}}$ where $F_{h}: u \mathrm{G}_{B} \mapsto\left[\left(u, P\left(\pi^{-1}(u) h\right)\right)\right], G_{A} \times_{G_{B}} \mathcal{H}_{B} \rightarrow G_{A} / G_{B}$ (see [3]). The translation of Theorem 5.14 in the above situation is that $K^{\pi}=\widetilde{\Delta}_{K^{\pi}}^{*} Q_{\mathcal{H}_{A}}^{\mathbf{C}}$ where $\widetilde{\Delta}_{K^{\pi}}=\left(\tilde{\delta}_{K^{\pi}}, \tilde{\zeta}_{K^{\pi}}\right)$ with $\tilde{\delta}_{K^{\pi}}=\pi_{A} \times \operatorname{id}_{\mathcal{H}_{B}}$ and $\tilde{\zeta}_{K^{\pi}}=\left(\pi_{A}\right)_{q}$. In particular the morphism $\left(\pi_{A} \times \operatorname{id}_{\mathcal{H}_{B}},\left(\pi_{A}\right)_{q}\right)$ is a (extremal) morphism between the kernels $K^{\pi}$ and $Q_{\mathcal{H}_{A}}^{\mathbf{C}}$.

To go further in this direction, note that it has been proven in [3] that the representation of $G_{A}$ on $\mathcal{H}_{A}$ can be realized as multiplication of the elements of $G_{A}$ on the Hilbert space $\mathcal{H}^{K}$. This realization is implemented by the formula

$$
\pi_{A}(u)=\gamma^{*} \circ(u \cdot) \circ \gamma \quad\left(u \in G_{A}\right)
$$

In this sense, and according to what has been indicated formerly, one can say that $\pi_{A}$ is the pull-back of the natural multiplication (collineation) operator associated with the Stiefel bundle $\mathcal{G} \times \mathcal{G}(p) \operatorname{Ran} p \rightarrow \mathcal{G} / \mathcal{G}(p)$.

Finally, as our last application here, we show next a similar result for completely positive mappings. So let us assume once again the setting of subsection 1.4. Then $\Phi(a)=V^{*} \circ \pi_{A}(a) \circ V,(a \in A)$, where $\Phi: A \rightarrow \mathcal{B}\left(\mathcal{H}_{0}\right)$ 
is a unital completely positive mapping, $\pi_{A}: A \rightarrow \mathcal{B}\left(\mathcal{H}_{A}\right)$ is a Stinespring dilation of $\Phi$, and $V: \mathcal{H}_{0} \rightarrow \mathcal{H}_{A}$ is the corresponding isometry between the Hilbert spaces $\mathcal{H}_{0}$ and $\mathcal{H}_{A}$. Note that $V\left(\mathcal{H}_{0}\right)$ is a Hilbert subspace of $\mathcal{H}_{A}$. Let $\Phi_{V\left(\mathcal{H}_{0}\right)}$ denote the unital, completely positive mapping

$$
\Phi_{V\left(\mathcal{H}_{0}\right)}: T \mapsto p_{V\left(\mathcal{H}_{0}\right)} \circ T \circ \iota_{V\left(\mathcal{H}_{0}\right)}, \mathcal{B}\left(\mathcal{H}_{A}\right) \rightarrow \mathcal{B}\left(V\left(\mathcal{H}_{0}\right)\right)
$$

associated with $V\left(\mathcal{H}_{0}\right)$ as in Proposition 4.4.

Lemma 6.3 In the above notation we have $\Phi(a)=V^{*} \circ \Phi_{V\left(\mathcal{H}_{0}\right)}\left(\pi_{A}(a)\right) \circ V$ for every $a \in A$.

Proof. First note that there exists the composition $\iota_{V\left(\mathcal{H}_{0}\right)} \circ V$ and

$$
V^{*}=\left(\iota_{V\left(\mathcal{H}_{0}\right)} \circ V\right)^{*}=V^{*} \iota_{V\left(\mathcal{H}_{0}\right)}^{*}=V^{*} p_{V\left(\mathcal{H}_{0}\right)} .
$$

Hence, for each $a \in A$,

$$
\begin{aligned}
V^{*} \Phi_{V\left(\mathcal{H}_{0}\right)}\left(\pi_{A}(a)\right) V & \left.=V^{*}\left(p_{V\left(\mathcal{H}_{0}\right)}\right) \pi_{A}(a) \iota_{V\left(\mathcal{H}_{0}\right)}\right) V \\
& =\left(V^{*} p_{V\left(\mathcal{H}_{0}\right)}\right) \pi_{A}(a)\left(\iota_{V\left(\mathcal{H}_{0}\right)} V\right) \\
& =V^{*} \pi_{A}(a) V \\
& =\Phi(a)
\end{aligned}
$$

as we wanted to show.

Looking at $\Phi$ and $\Phi_{V\left(\mathcal{H}_{0}\right)} \circ \pi_{A}$ as sections of appropriate trivial bundles, as in Example 3.3, Lemma 6.3 says that $\Phi$ can be regarded as the pull-back of $\Phi_{V\left(\mathcal{H}_{0}\right)} \circ \pi_{A}$. Moreover, we have the following result.

Proposition 6.4 The pair $\left(\pi_{A}, V\right)$ is a (extremal, with $M=1$ ) morphism in the category $\boldsymbol{C P o s}$ from $\Phi$ into $\Phi_{V\left(\mathcal{H}_{0}\right)}$.

Proof. Let $p$ be the orthogonal projection from $\mathcal{H}_{A}$ onto $\mathcal{H}_{B}$. Take $b$ in $B$. We know that $\pi_{A}(b) \in\{p\}^{\prime}$, see subsection 1.4. Thus $E_{p} \pi_{A}(b)=$ $p \pi_{A}(b) p+(1-p) \pi_{A}(b)(1-p)=\pi_{A}(b)[p+(1-p)]=\pi_{A}(b)$. By Lemma 6.3 we get $V \Phi(a)=\Phi_{V\left(\mathcal{H}_{0}\right)}\left(\pi_{A}(a)\right)$ for all $a \in A$. In fact, for every $x_{0}, y_{0} \in \mathcal{H}_{0}$,

$$
\begin{aligned}
\left(V \Phi(a) x_{0} \mid V y_{0}\right)_{V\left(\mathcal{H}_{0}\right)} & =\left(\Phi(a) x_{0} \mid y_{0}\right)_{\mathcal{H}_{A}}=\left(V^{*} \Phi_{V\left(\mathcal{H}_{0}\right)} V x_{0} \mid y_{0}\right)_{\mathcal{H}_{A}} \\
& =\left(\Phi_{V\left(\mathcal{H}_{0}\right)} V x_{0} \mid V y_{0}\right)_{V\left(\mathcal{H}_{0}\right)} .
\end{aligned}
$$

In particular the equality holds for every $b \in B \subseteq A$. Consequently we have shown that $\left(\pi_{A}, V\right)$ is a morphism in the category StLHer, see after 
Lemma 1.9 in subsection 1.4. Now, for every integer $n, h_{1}, \ldots, h_{n} \in \mathcal{H}_{0}$ and a matrix $\left(a_{i j}\right) \subseteq M_{n}(A)$ we have

$$
\begin{aligned}
\sum_{i, j=1}^{n}\left(\Phi_{V\left(\mathcal{H}_{0}\right)}\left(\pi_{A}\left(a_{i j}\right)\right) V h_{j} \mid V h_{i}\right)_{V\left(\mathcal{H}_{0}\right)} & =\sum_{i, j=1}^{n}\left(\left(V^{*} \Phi_{V\left(\mathcal{H}_{0}\right)}\left(\pi_{A}\left(a_{i j}\right) V\right) h_{j} \mid V h_{i}\right)_{\mathcal{H}_{A}}\right. \\
& =\sum_{i, j=1}^{n}\left(\Phi\left(a_{i j}\right) h_{j} \mid h_{i}\right)_{\mathcal{H}_{A}} .
\end{aligned}
$$

This means that, in fact, $\left(\pi_{A}, V\right)$ is a morphism in CPos .

In summary, in what concerns completely positive mappings, it has been shown in the paper that they can be viewed as objects in a category, called here CPos . Proposition 6.4 says furthermore that completely positive mappings in that category are in fact the pull-back of canonical objects, which are universal in this sense, and are the completely positive mappings associated to Grassmannian tautological bundles, as given by Proposition 4.4.

\section{Appendix: List of categories}

LHer (end of subsection 1.1); GrLHer (Definition 1.7); RepGLH (Definition 1.8); HomogLHer (subsection 1.3); StLHer (after Remark 1.10); Kern (Definition 2.4); Hilb (Definition 2.6); Trans, sRep, CPos (Remark 2.7).

\section{References}

[1] Alpay, D. and Levanony, D.: On the reproducing kernel Hilbert spaces associated with the fractional and bi-fractional Brownian motions. Potential Anal. 28 (2008), 163-184.

[2] Aronszajn, N.: Theory of reproducing kernels. Trans. Amer. Math. Soc. 68 (1950), 337-404.

[3] Beltiţă, D. and Galé, J. E.: Holomorphic geometric models for representations of $C^{*}$-algebras. J. Funct. Anal. 255 (2008), 2888-2932.

[4] Beltiţă, D. and Galé, J. E.: On complex infinite-dimensional Grassmann manifolds. Complex Anal. Oper. Theory 3 (2009), no. 4, 739-758.

[5] Beltiţă, D. and Ratiu, T. S.: Geometric representation theory for unitary groups of operator algebras. Adv. Math. 208 (2007), no. 1, 299-317.

[6] Bertram, W. and Hilgert, J.: Reproducing kernels on vector bundles. In Lie Theory and Its Applications in Physics III, 43-58. World Scientific, Singapore, 1998. 
[7] Carmeli, C., De Vito, E., Toigo, A. and Umanità, V.: Vector valued reproducing kernel Hilbert spaces and universality. Anal. Appl. (Singap.) 8 (2010), no. 1, 19-61.

[8] Choi, M. D. And Effros, E. G.: Injectivity and operator spaces. J. Functional Analysis 24 (1977), no. 2, 156-209.

[9] Dupré, M. J., Evard, J.-C. And Glazebrook, J. F.: Smooth parametrization of subspaces in a Banach space. Rev. Un. Mat. Argentina $4 \mathbf{1}$ (1998), no. 2, 1-13.

[10] Dupré, M. J. and Glazebrook, J. F.: The Stiefel bundle of a Banach algebra. Integral Equations Operator Theory 41 (2001), no. 3, 264-287.

[11] Dupré, M.J. And Glazebrook, J.F.: Holomorphic framings for projections in a Banach algebra. Georgian Math. J. 9 (2002), no. 3, 481-494.

[12] Effros, E. G. And RuAn, Zh.-J.: Operator Spaces. London Mathematical Society Monographs. New Series, 23. The Clarendon Press, Oxford University Press, New York, 2000.

[13] Faraut, J. and Thomas, E. G. F.: Invariant Hilbert spaces of holomorphic functions. J. Lie Theory 9 (1999), no. 2, 383-402.

[14] Galé, J. E.: Geometría de órbitas de representaciones de grupos y álgebras promediables. Rev. R. Acad. Cienc. Exactas Fís. Quím. Nat. Zaragoza (2) 61 (2006), 7-46.

[15] Halmos, P.R.: A Hilbert space problem book (second edition, revised and enlarged). Graduate Texts in Mathematics 19. Springer-Verlag, New York-Heidelberg-Berlin, 1982.

[16] Kobayashi, S.: Irreducibility of certain unitary representations. J. Math. Soc. Japan 20 (1968), 638-642.

[17] Krantz, S. G.: A tale of three kernels. Complex Var. Elliptic Equ. 53 (2008), no. 11, 1059-1082.

[18] Krantz, S. G.: On a construction of L. Hua for positive reproducing kernels. Michigan Math. J. 59 (2010), no. 1, 211-230.

[19] Kunze, R.: Positive definite operator valued kernels and unitary representations. In Functional Analysis (Proc. Conf., Irvine, Calif., 1966), 235-247. Academic Press, London; Thompson Book, Washington, D.C., 1967.

[20] Martin, M. and Salinas, N.: Flag manifolds and the Cowen-Douglas theory. J. Operator Theory 38 (1997), no. 2, 329-365.

[21] LANG, S.: Fundamentals of differential geometry (corrected 2nd printing). Graduate Texts in Mathematics 191. Springer-Verlag, New-York, 2001.

[22] Monastyrski, M. and Pasternak-Winiarski, Z.: Maps on complex manifolds into Grassmann spaces defined by reproducing kernels of Bergman type. Demonstratio Math. 30 (1997), no. 2, 465-474.

[23] Neeb, K.-H.: Holomorphy and convexity in Lie theory. De Gruyter Expositions in Mathematics 28. Walter de Gruyter, Berlin, 2000. 
[24] OdziJewicz, A.: On reproducing kernels and quantization of states. Comm. Math. Phys. 114 (1988), no. 4, 577-597.

[25] OdziJewicz, A.: Coherent states and geometric quantization. Comm. Math. Phys. 150 (1992), no. 2, 385-413.

[26] Paulsen, V.: Completely bounded maps and operator algebras. Cambridge Studies in Advanced Mathematics 78. Cambridge University Press, Cambridge, 2002.

[27] Raeburn, I.: The relationship between a commutative Banach algebra and its maximal ideal space. J. Functional Analysis 25 (1977), no. 4, 366-390.

[28] Upmeier, H.: Symmetric Banach Manifolds and Jordan $C^{*}$-algebras. North-Holland Mathematics Studies 104. Notas de Matemática 96. NorthHolland Publishing Co., Amsterdam, 1985.

[29] Wells, R. O., JR.: Differential analysis on complex manifolds (second edition). Graduate Texts in Mathematics 65. Springer-Verlag, New YorkBerlin, 1980.

[30] Zuccante, R.: Completely positive maps of the Cuntz algebras. J. Funct. Anal. 145 (1997), no. 1, 35-43.

Recibido: 12 de marzo de 2009

Daniel Beltiţă

Institute of Mathematics "Simion Stoilow"

Romanian Academy

P.O. Box 1-764, Bucharest, Romania

Daniel.Beltita@imar.ro

José E. Galé

Departamento de Matemáticas and I.U.M.A.

Universidad de Zaragoza

50009 Zaragoza, Spain

gale@unizar.es

This research has been partly supported by Project MTM2007-61446, DGI-FEDER, of the MCYT, Spain. The second-named author has also been supported by Project E-64, D.G. Aragón, Spain, while the first-named author acknowledges partial financial support from the CNCSIS grant PNII - Programme "Idei" (code 1194). 